Portland State University

PDXScholar

6-13-1995

\title{
Synthesis and Characterization of a Porphyrin Dyad
}

Dale A. Braden

Portland State University

Follow this and additional works at: https://pdxscholar.library.pdx.edu/open_access_etds

Part of the Chemistry Commons

Let us know how access to this document benefits you.

\section{Recommended Citation}

Braden, Dale A., "Synthesis and Characterization of a Porphyrin Dyad" (1995). Dissertations and Theses.

Paper 4976.

https://doi.org/10.15760/etd.6852

This Thesis is brought to you for free and open access. It has been accepted for inclusion in Dissertations and Theses by an authorized administrator of PDXScholar. Please contact us if we can make this document more accessible: pdxscholar@pdx.edu. 


\section{THESIS APPROVAL}

The abstract and thesis of Dale A. Braden for the Master of Science degree in

Chemistry were presented June 13,1995 and accepted by the thesis committee and the department.

COMMITTEE APPROVALS:

Carl C. Wamser, Chair

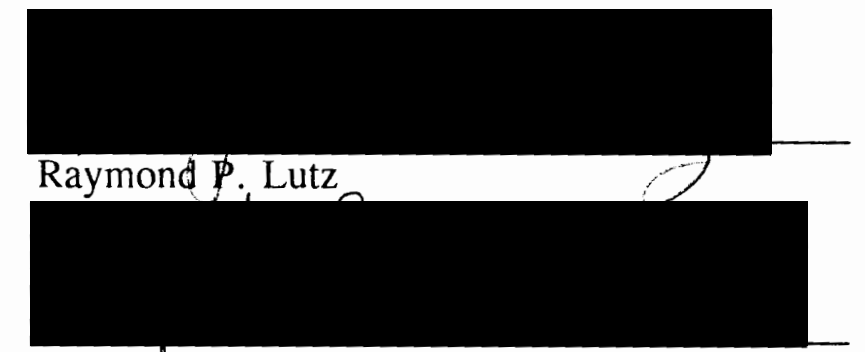

Alfred \$. Levinson

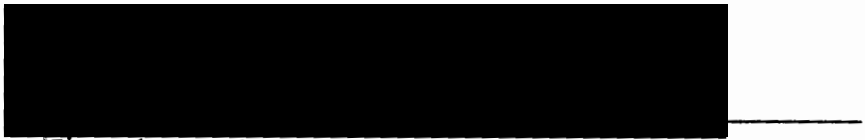

Jonathan Abramson

Representative of the Office of Graduate Studies

DEPARTMENT APPROVAL:

David H. Peyton, Chair

Department of Chemistry

ACCEPTED FOR PORTLAND STATE UNIVERSITY BY THE LIBRARY

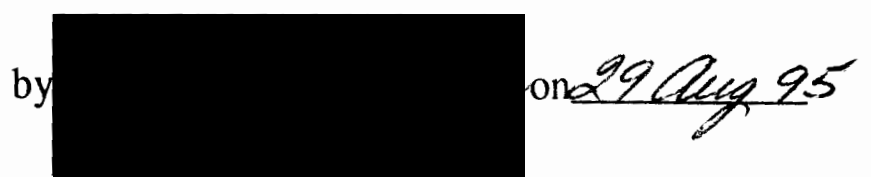




\begin{abstract}
An abstract of the thesis of Dale A. Braden for the Master of Science in Chemistry presented June 13, 1995.
\end{abstract}

Title: Synthesis and Characterization of a Porphyrin Dyad.

The sun is a bountiful source of energy for our planet. .With the advent of photovoltaic cells, man has begun harnessing the sun's radiant energy, turning it into a form more directly useful: electricity. Commercially available solar cells currently operate at about $13 \%$ efficiency, sufficiently high to make them a viable source of electrical energy. It is of great interest, however, to improve their conversion efficiency, and to lower the cost of production so as to make them more economical, and thereby reduce our dependence upon traditional "dirty" sources of energy such as coal and oil.

It has been found that an electrode coated with a thin film of nanocrystalline titanium dioxide, a very inexpensive and commonly available semiconductor, can be sensitized with a strong light-absorbing dye which can absorb the energy of sunlight and then transfer this energy as electronic charge into the electrode. A cell containing such an electrode is capable of producing a photocurrent at an appreciable voltage. 
The search is on to find the best sensitizing dye. It must absorb as much of the incident sunlight as possible, be capable of strong adsorption onto $\mathrm{TiO}_{2}$ so as to promote electron injection into the semiconductor, be relatively cheap and easy to synthesize, and be photochemically stable. It was the intent of this research to synthesize and test such a dye, a porphyrin dyad. The dyad was to be made from an electron donating moiety, meso-tetrakis(4-aminophenyl)porphyrin (TAPP), linked by an amide bond to an electron acceptor, meso-tetrakis(4-carboxyphenyl)porphyrin (TCPP). This material eluded attempts at synthesis, due to the poor reactivity of the aminoporphyrin and to the difficulty in ensuring that only one amide bond formed. Characterization of the monomers was carried out, and conditions for their chromatographic separation were determined. Recommendations for successful synthesis of the dyad are given. 


\title{
SYNTHESIS AND CHARACTERIZATION \\ OF A PORPHYRIN DYAD
}

\author{
by
}

Dale A. Braden

A thesis submitted in partial fulfillment of the requirements for the degree of

\section{MASTER OF SCIENCE \\ in \\ CHEMISTRY}

Portland State University

1995 


\section{ACKNOWLEDGEMENTS}

First and foremost, sine quo non, I thank Dr. Carl Wamser for his steady patience in the face of my incessant importunities, for his genuine care, guidance, friendship, and for his gifts of knowledge.

His research group was always a source of help and friendship as well:

James Weinkauf, Tristan Jenkins, Suman Cherian, Judith Lebzelter and Chang-Hwa Ryu.

I would also like to thank Dr. Gary Gard, Dr. Nick Hamel and Steven Ullrich for giving me my start in laboratory chemistry.

I am grateful to Dr. David Peyton and Clary Clish for helping me obtain NMR spectra (on more than one occasion!); to Lorne Isabelle at the Oregon Graduate Institute for acquiring FAB mass spectra; to Dr. Lilo Barofsky at Oregon State University for acquiring MALDI mass spectra; and again to Judith Lebzelter for helping me with computer software and instrumentation. 
TABLE OF CONTENTS

PAGE

ACKNOWLEDGEMENTS $\ldots \ldots \ldots \ldots \ldots \ldots \ldots \ldots$. . . . . . . . . .

LIST OF FIGURES $\ldots \ldots \ldots \ldots \ldots \ldots \ldots \ldots \ldots \ldots \ldots \ldots \ldots \ldots \ldots$

CHAPTER

I SOLAR ENERGY CELLS $\ldots \ldots \ldots \ldots \ldots \ldots \ldots \ldots$

Introduction $\ldots \ldots \ldots \ldots \ldots \ldots \ldots \ldots \ldots$

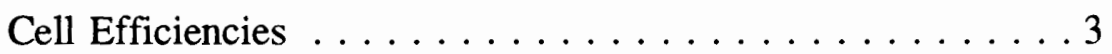

Solar Cells in General . . . . . . . . . . 3

Photosynthesis . . . . . . . . . . 10

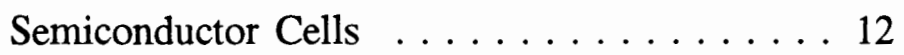

The Dye-Sensitized Semiconductor Cell . . . . . . . . . 13

Photoinduced Electron Transfer . . . . . . . 13

Sensitization of Semiconductors $\ldots \ldots \ldots 18$

The Grätzel Cell ............. 21

II RESULTS AND DISCUSSION $\ldots \ldots \ldots \ldots \ldots \ldots \ldots$

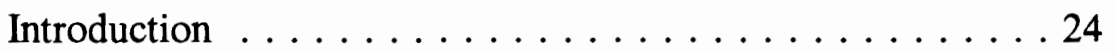

Attempted Syntheses of the TCPP-TAPP Dyad . . . . . 28

General Approach ............ 28

Synthesis Using a Merrifield Resin . . . . . 37 
Synthesis on $\mathrm{TiO}_{2}$ Colloids . . . . . . . 40

Synthesis from Methylated TCPP . . . . . 41

Spectroscopy $\ldots \ldots \ldots \ldots \ldots \ldots \ldots \ldots \ldots$

Ultraviolet/Visible Spectroscopy . . . . . . . 46

Fluorescence Spectroscopy . . . . . . . 48

NMR Spectroscopy $\ldots \ldots \ldots \ldots \ldots \ldots 4$

Chromatography .................. 55

Conclusions $\ldots \ldots \ldots \ldots \ldots \ldots \ldots \ldots$

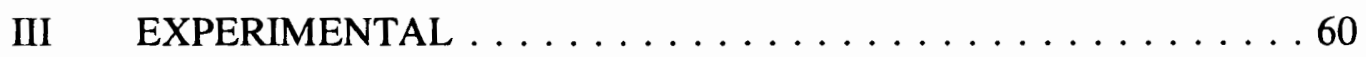

Materials and Methods $\ldots \ldots \ldots \ldots \ldots \ldots 60 \ldots$

Instrumentation $\ldots \ldots \ldots \ldots \ldots \ldots \ldots$

Synthesis of terephthalanilic acid (I) $\ldots \ldots \ldots \ldots 62$

Synthesis of terephthalanilic acid (II) . . . . . . 63

Synthesis of TCPP-TAPP dyad (DCC method) . . . . . . 64

TCPP-TAPP linkage using a Merrifield resin $\ldots \ldots \ldots 66$

Test of cleavage of TCPP from Merrifield resin $\ldots \ldots 68$

Adsorption of TCPP onto $\mathrm{TiO}_{2} \ldots \ldots \ldots 69$

Addition of TAPP to $\mathrm{TiO}_{2}$ treated with $\mathrm{SOCl}_{2} \ldots \ldots 70$

TCPP-TAPP linkage on $\mathrm{TiO}_{2}$ using $\mathrm{SOCl}_{2} \ldots \ldots \ldots 71$

TCPP-TAPP linkage on $\mathrm{TiO}_{2}$ using diisopropylcarbodiimide 71

TCPP-TAPP linkage on $\mathrm{TiO}_{2}$ using $\phi_{3} \mathrm{P}$ and $\mathrm{CCl}_{4} \ldots \ldots 72$

TCPP-TAPP linkage on $\mathrm{TiO}_{2}$ via pyrolysis $\ldots \ldots \ldots 73$ 
TCPP-TAPP coupling with diisopropylcarbodiimide (DIC) 73 Synthesis of TCPP-tetraacid chloride (TCCPP) . . . . . . 74 TCPP-TAPP polymer $\ldots \ldots \ldots \ldots \ldots \ldots$

Synthesis of TCPP-methyl esters $\ldots \ldots \ldots \ldots \ldots 75$

Synthesis of TCPP-TAPP dyad from TCCPP . . . . . 76

Synthesis of TCPP-TAPP dyad from TrMCPP . . . . . 79

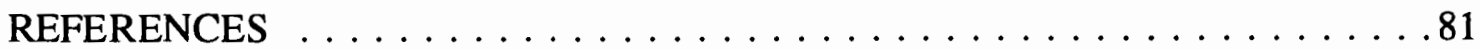

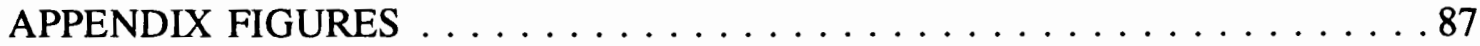




\section{LIST OF FIGURES}

FIGURE

PAGE

1. Plots of cell efficiency versus bandgap wavelength $\ldots \ldots \ldots \ldots \ldots$

2. Energy state diagram showing various states attainable

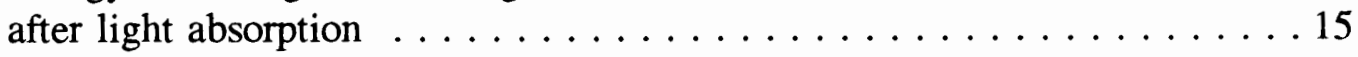

3. Schematic diagram of a photovoltaic cell using a dyesensitized semiconductor electrode . . . . . . . . . . . . . . . 19

4. The synthetic goal: the TCPP-TAPP dyad $\ldots \ldots \ldots \ldots \ldots$

5. Energy state diagram for the TCPP-TAPP dyad $\ldots \ldots \ldots 27$

6. Structures and abbreviations for the porphyrins

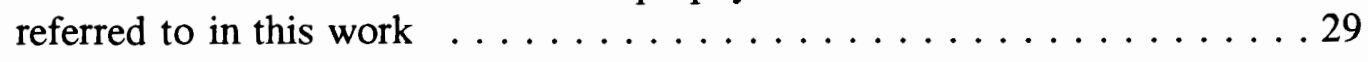

7. Reaction pathways for carbodiimide coupling $\ldots \ldots \ldots \ldots \ldots$

8. $\quad 500 \mathrm{MHz}{ }^{1} \mathrm{H}$ NMR spectrum of product of DCC coupling of terephthalic acid and aniline, in DMSO- $\mathrm{d}_{6} \ldots \ldots \ldots \ldots \ldots \ldots \ldots$

9. $500 \mathrm{MHz}{ }^{1} \mathrm{H}$ NMR spectrum of benzanilide in DMSO- $\mathrm{d}_{6} \ldots \ldots \ldots 33$

10. $400 \mathrm{MHz}{ }^{1} \mathrm{H}$ NMR spectrum of the product mixture from DIC

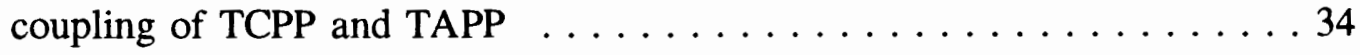

11. $500 \mathrm{MHz}{ }^{1} \mathrm{H}$ NMR spectrum of TAPP in DMSO-d $\mathrm{d}_{6} \ldots \ldots \ldots 35$

12. $500 \mathrm{MHz}{ }^{1} \mathrm{H}$ NMR spectrum of TCPP in DMSO- $\mathrm{d}_{6} \ldots \ldots \ldots \ldots$

13. Synthetic scheme for the TCPP-TAPP dyad using a Merrifield resin . . . . . . . . . . . . . . . . . . . . 39

14. MALDI mass spectrum of the products of reaction of TAPP with a TCPP acid chloride 
15. Absorption spectrum of an equimolar mixture of TCPP and TAPP in DMSO

16. Emission spectra of TCPP and TAPP solutions

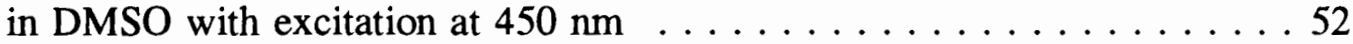

17. Emission spectra of TCPP and TAPP solutions in DMSO with excitation at $416 \mathrm{~nm} \ldots \ldots \ldots \ldots \ldots \ldots \ldots$

18. Absorption spectrum of TAPP in acetone $\ldots \ldots \ldots \ldots \ldots$

19. Absorption spectrum of TAPP in DMF $\ldots \ldots \ldots \ldots \ldots \ldots$

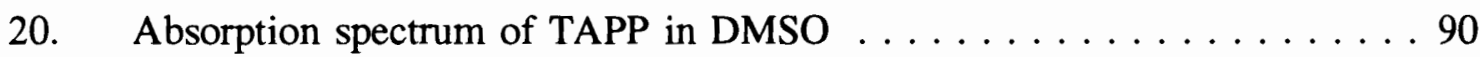

21. Absorption spectrum of TAPP in methanol $\ldots \ldots \ldots \ldots \ldots 1$

22. Absorption spectrum of TAPP in $1.2 \%$ aqueous pyridine $\ldots \ldots \ldots 92$

23. Absorption spectrum of TAPP in water w/ $0.5 \%$ DMSO $\ldots \ldots \ldots 93$

24. Absorption spectrum of TCPP in acetone $\ldots \ldots \ldots \ldots \ldots 4$

25. Absorption spectrum of TCPP in DMF $\ldots \ldots \ldots \ldots \ldots$

26. Absorption spectrum of TCPP in DMSO ............. 96

27. Absorption spectrum of TCPP in methanol ............ 97

28. Absorption spectrum of TCPP in $2 \mathrm{M} \mathrm{NaCl} \ldots \ldots \ldots \ldots$

29. Absorption spectrum of TCPP in $1.2 \%$ aqueous pyridine $\ldots \ldots \ldots \ldots 99$

30. Absorption spectrum of TMCPP in DMSO $\ldots \ldots \ldots \ldots \ldots$

31. Absorption spectrum of TMCPP in dichloromethane $\ldots \ldots \ldots \ldots 101$

32. Excitation spectrum of TAPP in acetone $\ldots \ldots \ldots \ldots 2 \ldots \ldots \ldots$

33. Excitation spectrum of TAPP in dichloromethane $\ldots \ldots \ldots \ldots$

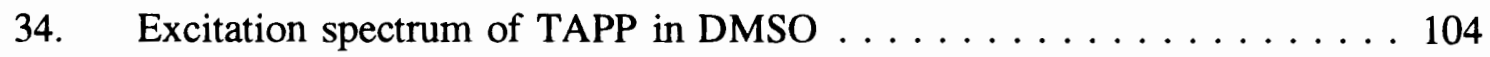

35. Excitation spectrum of TAPP in ethyl acetate $\ldots \ldots \ldots \ldots$ 
36. Excitation spectrum of TAPP in $1 \mathrm{M} \mathrm{HCl} \ldots \ldots \ldots 106$

37. Excitation spectrum of TAPP in methanol $\ldots \ldots \ldots \ldots \ldots$

38. Excitation spectrum of TAPP in $1.2 \%$ aqueous pyridine $\ldots \ldots \ldots 108$

39. Excitation spectrum of TCPP in acetone . . . . . . . . . . 109

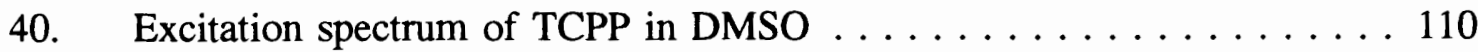

41. Excitation spectrum of TCPP in ethyl acetate $\ldots \ldots \ldots \ldots \ldots 111$

42. Excitation spectrum of TCPP in $1 \mathrm{M} \mathrm{HCl} \ldots \ldots \ldots \ldots \ldots \ldots 112$

43. Excitation spectrum of TCPP in methanol $\ldots \ldots \ldots \ldots \ldots \ldots$

44. Excitation spectrum of TCPP in $1.2 \%$ aqueous pyridine $\ldots \ldots \ldots 114$

45. Excitation spectrum of TMCPP in dichloromethane $\ldots \ldots \ldots \ldots 115$

46. Excitation spectrum of TMCPP in DMSO $\ldots \ldots \ldots \ldots \ldots \ldots$

47. Emission spectrum of TAPP in acetone $\ldots \ldots \ldots \ldots \ldots \ldots \ldots$

48. Emission spectrum of TAPP in dichloromethane $\ldots \ldots \ldots \ldots \ldots 118$

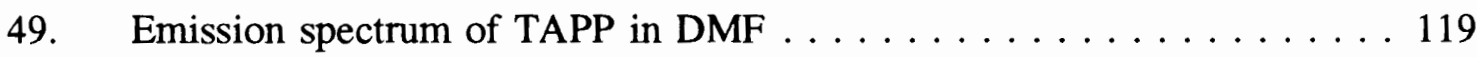

50. Emission spectrum of TAPP in DMSO $\ldots \ldots \ldots \ldots \ldots \ldots$

51. Emission spectrum of TAPP in ethyl acetate $\ldots \ldots \ldots \ldots \ldots \ldots 121$

52. Emission spectrum of TAPP in $1 \mathrm{M} \mathrm{HCl} \ldots \ldots \ldots \ldots \ldots \ldots \ldots$

53. Emission spectrum of TAPP in methanol $\ldots \ldots \ldots \ldots \ldots \ldots \ldots$

54. Emission spectrum of TAPP in $1.2 \%$ aqueous pyridine $\ldots \ldots \ldots 124$

55. Emission spectnum of TCPP in acetone $\ldots \ldots \ldots \ldots \ldots \ldots \ldots$

56. Emission spectrum of TCPP in DMF $\ldots \ldots \ldots \ldots \ldots \ldots \ldots \ldots$

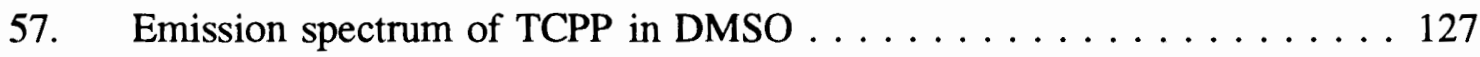


58. Emission spectrum of TCPP in ethyl acetate $\ldots \ldots \ldots \ldots \ldots$

59. Emission spectrum of $\mathrm{TCPP}$ in $1 \mathrm{M} \mathrm{HCl} \ldots \ldots \ldots \ldots$

60. Emission spectrum of TCPP in methanol $\ldots \ldots \ldots \ldots \ldots$

61. Emission spectrum of TCPP in $1.2 \%$ aqueous pyridine $\ldots \ldots \ldots 131$

62. Emission spectrum of TMCPP in dichloromethane $\ldots \ldots \ldots \ldots 132$

63. Emission spectrum of TMCPP in DMSO $\ldots \ldots \ldots \ldots \ldots$

64. $500 \mathrm{MHz}{ }^{1} \mathrm{H}$ NMR spectrum of TAPP in DMSO-d $\mathrm{d}_{6} \ldots \ldots \ldots$

65. $500 \mathrm{MHz}^{1} \mathrm{H}$ NMR spectrum of $\mathrm{TAPP}+\mathrm{D}_{2} \mathrm{O}$ in $\mathrm{DMSO}-\mathrm{d}_{6} \ldots \ldots$

66. $500 \mathrm{MHz}^{1} \mathrm{H}$ NMR spectrum of TCPP in DMSO-d $\ldots \ldots \ldots$

67. $500 \mathrm{MHz}{ }^{1} \mathrm{H}$ NMR spectrum of $\mathrm{TCPP}+\mathrm{D}_{2} \mathrm{O}$ in $\mathrm{DMSO}-\mathrm{d}_{6} \ldots \ldots 137$ 


\section{CHAPTER I}

\section{SOLAR ENERGY CELLS}

\section{INTRODUCTION}

The sun has long been recognized as an important source of exploitable energy. It is an inexhaustible source as far as we are concerned, its light energy is available almost everywhere, and it can be tapped without tremendous destruction or dangerous alteration to the environment, which makes its use preferable to that of burning oil, coal or wood, and to flooding valleys behind hydroelectric dams in order to use water. We have already seen success in the area of solar energy utilization in the form of solar panels that heat many modern homes and power satellites, and in the form of the solar cells that run certain calculators. Photothermal converters are inherently more efficient than photovoltaic converters ${ }^{1}$, but since electricity is a more directly useful form of energy, photovoltaic (PV) cells are receiving intense study. Applied research is aimed at developing ever more efficient cells, while keeping them small, lightweight and as nontoxic and inexpensive to manufacture as possible.

Most commercial solar cells are based upon p-n junctions between doped or alloyed semiconductors ${ }^{2-4}$. The primary drawback to these cells is their high manufacturing $\operatorname{cost}^{4}$. In the case of gallium arsenide cells, which are the most efficient of all, toxicity is also a serious problem. These problems are largely 
avoided, at the expense of some energy conversion efficiency, for cells based upon dye-sensitized metal oxide semiconductor electrodes. Many metal oxide semiconductors, especially $\mathrm{TiO}_{2}$, are quite inexpensive, readily available, and nontoxic, but they generally have high bandgaps and so absorb only a small fraction of sunlight. For this reason, it is desirable to sensitize them with a thin layer of an organic dye that absorbs strongly over as broad a wavelength range as possible. As long as the excited state of the sensitizer is higher in energy than the acceptor states in the semiconductor, then electron transfer to the semiconductor can take place, and the cell can produce an electric current. However, the amount of sensitizer that can be adsorbed on a flat surface is quite small, so that the total absorbance is still very low, less than $1 \%$ for the strongest absorbers ${ }^{5,6}$. Building up a thicker layer of sensitizer does not help, because the outer layers of dye do not efficiently transport charge through the inner layers ${ }^{5}$. This problem has been solved by using a polycrystalline film of the semiconductor composed of colloid-sized particles sintered together to provide electrical contact. The surface area of such a film is enhanced approximately 1000 -fold ${ }^{7}$, and the amount of dye that can be adsorbed is dramatically increased.

The choice of sensitizer is critical, for it must bond to the semiconductor in order to make electron transfer efficient, it must have good absorption properties in order to take in as much of the sun's energy as possible, and it must be photochemically stable so as not to break down with use. It is one choice of sensitizer, a porphyrin dyad, that was investigated in this research. 
Before discussing dye sensitization of semiconductor electrodes in more detail, it will be useful to discuss the maximum theoretically attainable efficiencies for solar cells in general. A comparison with the efficiency of nature's solar cells, the chloroplasts of photosynthesis, will also be illustrative.

\section{CELL EFFICIENCIES}

\section{$\underline{\text { Solar Cells in General }}$}

Since there is a definite energy difference, $E_{\delta}$, between the HOMO and LUMO levels for any compound (or between the conduction and valence bands of a semiconductor), an absorbed photon must usually possess at least this amount of energy in order for electronic excitation to take place. Thus, of the total light incident upon a cell, only that of wavelength $\leq \lambda_{\mathrm{g}}$, the threshold wavelength, can result in electronic excitation. But not all of this light will actually be absorbed: some will be reflected or scattered. Of the light that is absorbed by the cell, only part will actually be absorbed by the primary absorber, i.e., that species which is actually capable of conducting electrons, or transferring them into the electrode. Finally, since vibrational relaxation is a very fast process (typically $\left.\approx 10^{-12}-10^{-14} \mathrm{~s}\right)^{8}$, a molecule that has absorbed a photon of energy greater than $E_{g}$ will quickly relax to the lowest vibrational level of the electronically excited state, losing the excess energy as heat. Thus, for a solar cell based upon a single primary absorber, the fraction of the total incident energy that results in lowest-energy excited states is given by: ${ }^{9}$ 


$$
\eta_{a b s}=\frac{\int_{0}^{\lambda_{g}} I(\lambda)\left[\lambda / \lambda_{g}\right]\left[A(\lambda) / A_{t o t}(\lambda)\right]\left[1-10^{-A_{t o t}(\lambda)}\right] d \lambda}{\int_{0}^{\infty} I(\lambda) d \lambda}
$$

where $\lambda_{g}$ is the wavelength corresponding to $E_{g}$, and $I(\lambda)$ is the solar irradiance in $\mathrm{Wm}^{-2} \mathrm{~nm}^{-1}$. The term $\left[1-10^{- \text {Atot }(\lambda)}\right]$ is the fraction of incident light absorbed by the entire cell, $\left[A(\lambda) / A_{\text {tor }}(\lambda)\right]$ is the fraction of absorbed light that is absorbed by the primary absorber, and $\left[\lambda / \lambda_{g}\right]$ accounts for losses due to vibrational relaxation. Curve $A$ in Figure 1 shows a plot of $\eta_{\text {abs }}$ versus $\lambda_{\mathrm{g}}$ for a single perfect absorber, i.e., $A(\lambda)=$ $A_{\text {tor }}(\lambda)=\infty$. The values of $I(\lambda)$ were taken from standard solar irradiance data ${ }^{10}$. We see here the effect of energy losses due to vibrational relaxation: the curve maximum is only $49 \%$, and the optimum bandgap is $1100 \mathrm{~nm}$.

Only a fraction of the excited molecules will now transfer an electron; many lose their excitation energy as fluorescence or phosphorescence, or undergo internal conversion to the ground state. The quantum yield, $\Phi$, expresses the fraction of products, either electrons or reduced molecules, created per photon absorbed. It is normally assumed to be independent of the wavelength of absorbed light. Including this term will further reduce the efficiency, without changing the value of the optimum wavelength. The quantum yield for electron transfer from the excited state of the special pair of chlorophyll molecules in the photosynthetic reaction center is approximately unity ${ }^{11,12}$. Man-made systems have also achieved quantum yields this high $^{7,13}$. 


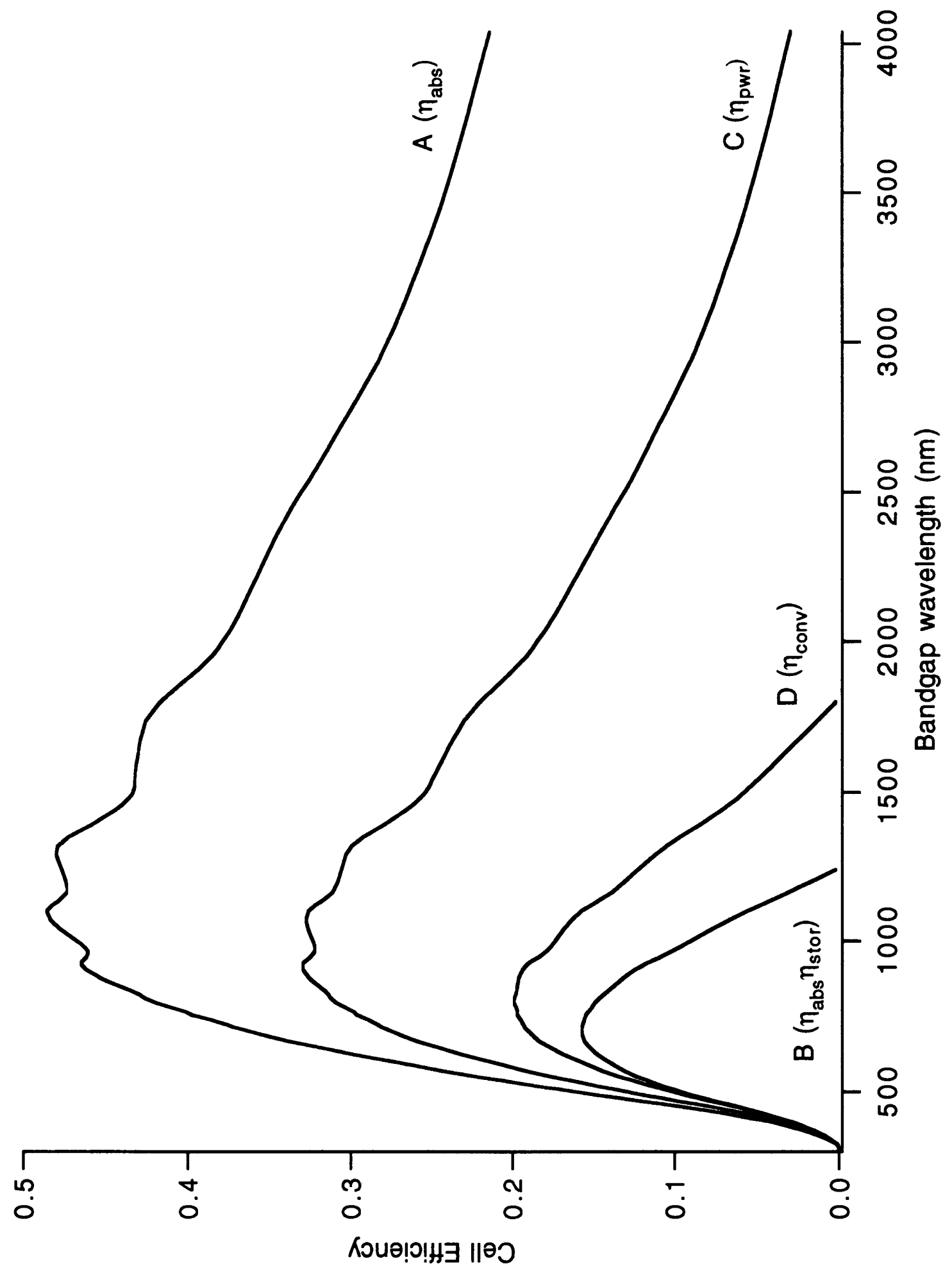

Figure 1. Plots of cell efficiency versus bandgap wavelength. 
The second law of thermodynamics requires that the products formed in the photochemical reaction preserve only a fraction of the excitation energy that creates them. Alternatively (but not equivalently), we may regard the lost energy as being used to establish a kinetic barrier, $E_{a}$, for the reverse reaction, which is desirable anyway if the cell is to store energy ${ }^{14}$. The free energy difference between photoproducts and reactants is $\Delta G=E_{g}-E_{a}$, and the fraction of excitation energy stored in the photoproducts is then $\Delta G / E_{g}=\eta_{\text {stor }}$. Inclusion of this term has a pronounced effect on the overall cell efficiency, as can be seen in Figure 1, curve $B$. Here, $E_{a}=1 \mathrm{eV}$, and $\Phi=1$. The peak efficiency is now only $16 \%$, and the most efficient wavelength is $710 \mathrm{~nm}$, very nearly that for photosystem I in chloroplasts.

Finally, we should realize that only a fraction of the photoproducts will be storable, in the case of reduced chemicals, or directly usable, in the case of photoelectrons. Some photoproducts will inevitably be lost through leakage or reaction with impurities in the cell, electron-hole recombination at boundaries, and so forth. These losses are accounted for by $\eta_{\text {coll }}$ in the following equation, which expresses the overall cell efficiency: ${ }^{9,14}$

$$
\eta_{\text {cell }}=\eta_{\text {abs }} \phi \eta_{\text {stor }} \eta_{\text {coll }}
$$

Ross and $\mathrm{Hsiao}^{15}$ have included the effect of inherent irreversibility of the photochemical reaction in their approach to the problem of determining maximum photovoltaic cell efficiencies. They considered the chemical potential difference between the ground state absorber molecules and the excited molecules, and found 
that the maximum possible difference occurs when the rate of excited state emission equals the rate of ground state absorption, i.e., when no electron transfer occurs at all and the cell does no work. They derived the following equation for the maximum chemical potential difference attainable by a cell based upon a single absorbing species:

$$
\mu_{\max } \approx \frac{h c}{\lambda_{g}}+k T \ln \int_{0}^{\lambda_{g}} N(\lambda) d \lambda-k T \ln \left(\frac{8 \pi n^{2} k T}{h \lambda_{g}^{2}}\right)
$$

where $N(\lambda)$ is the absorbed flux (in photons per square meter per second per unit of wavelength), and $n$ is the refractive index of the medium $\approx 1$. Bolton ${ }^{14,16}$ used this equation to calculate efficiency curves for solar cells. In a later review, Pirt ${ }^{17}$ showed that the Ross and Hsiao equation was formally identical to a much simpler one proposed in 1958 by Duysens ${ }^{18}$. Based on an original work by Spanner, Pirt also noted that a factor of $4 / 3$ should be placed in front of the logarithmic terms to account for the effect of radiation pressure ${ }^{17,19} . \mu_{\max }$ represents the greatest amount of excitation energy that a photon can induce, even assuming no radiative or nonradiative losses. Multiplying this by the total absorbed flux, and dividing by the total solar irradiance gives the power efficiency of the cell:

$$
\eta_{p w T}=\frac{\mu_{\max } \int_{0}^{\lambda_{g}} N(\lambda) \epsilon(\lambda) d \lambda}{\int_{0}^{\infty} I(\lambda) d \lambda}
$$


where $\epsilon(\lambda)$, the extinction coefficient for the absorbing species, is assumed here to be unity for all wavelengths. Curve $C$ in Figure 1 is a plot of $\eta_{p w r}$. It beautifully illustrates the effect of the second law: even when the cell does no work, the energy it produces (in the form of emitted radiation in this case) is still less than what went into it.

When the cell is required to do work, further energy losses result, both radiative and nonradiative. Ross and Hsiao estimated that the converted fraction of energy is then given by

$$
f \approx\left[1-\frac{k T}{\mu_{\max }}\right]\left[\mu_{\max }-k T \ln \left(\frac{\mu_{\max }}{k T}\right)-k T \ln \alpha\right]
$$

where $\alpha$ is the fraction lost via nonradiative processes. Assuming that $\alpha=1$ (no nonradiative losses), we replace $\mu_{\max }$ in Equation (4) by $f$ to calculate Curve $D$ ( $\eta_{\text {conv }}$ ) in Figure 1.

Curves like those in Figure 1 have been calculated by Ross and $\mathrm{Hsiao}^{15}$ for AM 0 radiation, and by Bolton ${ }^{14}$ for AM 1.2 radiation. Those here have been calculated for AM 1.5 radiation according to standard terrestrial irradiance data published by the American Society for Testing and Materials (ASTM), which "are representative of average conditions in the 48 contiguous states of the United States $^{\prime 10}$. The 'air mass' (AM) refers to the mass, or thickness, of the atmosphere through which sunlight passes before reaching the ground. The AM 0 spectrum is for light incident upon the outer atmosphere, AM 1 is for light passing through the 
atmosphere above the equator at noon. Due to the northern latitude of most of the United States, sunlight reaches us at an angle, and the total irradiance is therefore less than that falling on the equator. The AM 1.5 solar spectrum is thus appropriate for calculations of efficiencies for solar cells to be used in this country. For this reason, and because of the correction for radiation pressure mentioned above, the maximum efficiencies calculated here are somewhat different from those previously published $^{14,20,21}$. Curve $D$ shows that the maximum theoretical efficiency for any single bandgap solar converter (including a dye-sensitized semiconductor cell) operating under unconcentrated, global AM 1.5 sunlight is approximately $20 \%$, based upon the assumptions of Ross and Hsiao ${ }^{15}$. This is actually less than the measured efficiency for the best gallium arsenide cell (see below). The discrepancy may be due to the fact that ASTM values for the irradiance of the sun are not taken from field measurements, but are calculated based upon various atmospheric parameters such as the concentration of absorbing gases, particulates, and the pathlength of the atmosphere. Experimental cells are normally not tested under natural sunlight, but with a solar simulator as a light source. Although the total irradiance of a solar simulator may be close to that for the calculated value at a given air mass, the photon flux at a given wavelength may be quite different. Ross and Collins ${ }^{21}$ calculated a maximum efficiency of $33 \%$ using AM 1.5 radiation, but they used a value of $800 \mathrm{~W} / \mathrm{m}^{2}$ for the total solar irradiance; the standard value ${ }^{10}$ is $963.75 \mathrm{~W} / \mathrm{m}^{2}$. Their choice may reflect the AM 1.5 direct irradiance, as opposed to the higher AM 1.5 global value (direct plus diffuse), but they do not specify. Making this correction 
only in the denominator of Equation 4 would reduce their value to $28 \%$. All detailed balance calculations are ultimately dependent upon the values one chooses to use for solar irradiance. From this data one determines the photon flux over each interval of wavelength, and this in turn affects $\mu_{\max }$. Standard data have obviously changed over the years, and this affects the calculations. It is worth pointing out also that all of the above models assume that all light of energy greater than $E_{g}$ is absorbed; in reality this will never be true.

We also notice in Figure 1 that the most efficient bandgap wavelength decreases as more energy losses are accounted for, and approaches the absorption maximum for the primary absorbing pigments in photosynthesis.

\section{Photosynthesis}

Many efforts have been made to determine the efficiency of photosynthesis $^{17,22}$. We should point out that most of these calculate the efficiency based only upon utilization of photosynthetically active radiation (PAR), which is that between 400 and $700 \mathrm{~nm}$. In order to make a fair comparison with solar cells, we must base our calculations upon the total terrestrial irradiance, which is 963.75 $\mathrm{Wm}^{-2}$ for wavelengths between $305-4095 \mathrm{~nm}^{10}$. Ignoring all thermodynamic requirements on energy conversion, we see right away that plants are able to use only $43 \%$ of the energy of light incident upon them. In fact, only $75-90 \%$ of the PAR is actually absorbed ${ }^{23}$; the rest is reflected by the leaf.

For photosynthesis in both bacteria and green plants, the quantum yield is practically $100 \%$ if it is defined as referring to the number of electrons transferred 
per photon absorbed ${ }^{11,12}$. If it is defined as the number of product molecules (oxygen) generated per photon absorbed, then it is about $11 \%^{23}$ (see also ref. 17 for a figure of $17-20 \%]$.

Although some recent evidence has appeared which is incompatible with it ${ }^{17}$, the Z-scheme for plant photosynthesis is still considered accurate ${ }^{24}$. The Z-scheme ${ }^{25}$ assumes that two photosystems, called PS I and PS II, act together to transfer electrons from water, which is oxidized to molecular oxygen, to $\mathrm{NADP}^{+}$, which is reduced to NADPH. NADPH is then used to reduce $\mathrm{CO}_{2}$, among other things, and attach it to ribulose-5-bisphosphate, which then undergoes various reactions leading ultimately to glucose, which is usually considered to be the energy storage product for the entire photosynthetic process. Assuming the overall equation for photosynthesis to be:

$$
\mathrm{H}_{2} \mathrm{O}(\mathrm{l})+\mathrm{CO}_{2}(\mathrm{~g}) \rightarrow 1 / 6 \mathrm{C}_{6} \mathrm{H}_{12} \mathrm{O}_{6}(\mathrm{~s})+\mathrm{O}_{2}(\mathrm{~g})
$$

Bolton $^{22}$ used the following formula to calculate a maximum theoretical efficiency for photosynthesis:

$$
\eta=\frac{\frac{\Delta G}{N_{A}} \int_{0}^{\lambda_{g}} N(\lambda) d \lambda}{\int_{0}^{\infty} I(\lambda) d \lambda} \cdot \phi_{Q_{2}} \cdot \eta_{a} \cdot 100 \%=13 \%
$$

in which $\Delta G$ for the above reaction is $496 \mathrm{~kJ} / \mathrm{mol}, \lambda_{g}=700 \mathrm{~nm}, \Phi=0.125$, which assumes the Z-scheme requirement of 8 photons needed to transfer 4 electrons to 
produce one molecule of oxygen; and $\eta_{\mathrm{a}}$, the absorption efficiency, is unity. Using

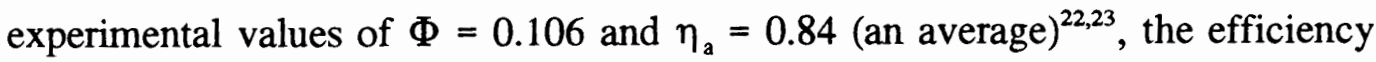
drops to $9 \%$. These calculations are based upon data obtained under laboratory conditions. In the field, photosynthetic efficiency is thought to be about $0.1 \%{ }^{24}$. Actually, the photosynthetic process is more accurately modelled as two connected engines: a photovoltaic cell which drives a chemical fuel pump ${ }^{26}$. The PV cell models the light reactions according to the Z-scheme, and produces highenergy electrons which the fuel pump uses (as NADPH) to produce glucose. If we want to compare photosynthesis with man-made PV cells, we should consider only the photovoltaic process, in which electrons are moved from water to $\mathrm{NADP}^{+}$using the energy of light. The oxidation potential of water is $+0.8 \mathrm{eV}$, and that for NADPH is $-0.32 \mathrm{eV}^{17}$. Since $\Delta G=-n F \Delta E$, the energy stored is $216 \mathrm{~kJ} / \mathrm{mol}$. The quantum yield is 0.5 here, because two electrons are transferred to $\mathrm{NADP}^{+}$for every four photons absorbed. Using these values in Equation (7), along with the experimental value of 0.84 for $\eta_{a}$, we obtain a value of $20 \%$.

\section{Semiconductor Cells}

Commercially available suiar cells are based upon p- and n-doped semiconductors. Light of the bandgap energy incident upon the p-n boundary causes electrons in the valence band of the semiconductor material to jump into the conduction band. These photoelectrons are attracted to the n-doped region, which is positively charged because of the diffusion of holes into it from the p-doped region. This constitutes the electric current generated by the cell under irradiation by 
sunlight. The most common semiconductor materials used in PV cells are amorphous silicon (a-Si), crystalline silicon (x-Si), copper indium diselenide $\left(\mathrm{CuInSe}_{2}\right)$, cadmium telluride (CdTe) and gallium arsenide ( $\left.\mathrm{GaAs}\right)$ and its alloys ${ }^{2,3}$. Cells based upon the last of these have attained the highest efficiencies so far, up to $25.7 \%$ for a single-junction cell under one-sun illumination ${ }^{3,27}$. GaAs cells have a theoretical maximum efficiency of $39 \%$ under concentrated sunlight of 1000 suns, if the angle of light acceptance by the cell is restricted ${ }^{28}$. Concentrating light on a semiconductor increases the strength of the electric field at the p-n junction, which increases the amount of charge that can be separated, and consequently the cell efficiency increases ${ }^{4}$.

Current research is focused upon multijunction devices. In cells of this type, different semiconductor materials are layered on top of each other in order by energy gap, with the material of largest energy gap on top. The maximum theoretical efficiency becomes $68 \%^{3}$ under unconcentrated sunlight, and $87 \%$ under concentration. In 1988, a cell with a top layer of GaAs and a bottom layer of crystalline silicon was produced by Varian which was $31.0 \%$ efficient ${ }^{29}$.

\section{THE DYE-SENSITIZED SEMICONDUCTOR CELL}

\section{Photoinduced Electron Transfer}

The process in which the absorption of light by a molecule results in transfer of an electron to another molecule is called photoinduced electron transfer (PET). A photon having the correct energy may be absorbed by a molecule, which then enters 
an electronically excited state (usually a singlet state) in which the electron is in a high-energy (usually antibonding) orbital. If this orbital is higher in energy than the lowest unoccupied molecular orbital (LUMO) of a neighboring molecule or of a covalently attached moiety, then the electron possesses a thermodynamic driving force to be transferred into the lower-energy orbital, giving rise to a charge transfer state. There are competing processes, however, which also serve to dissipate the excitation energy. The molecule can lose energy as fluorescence, transfer its excitation energy directly to another molecule, enter a triplet excited state (which involves loss of energy due to electron spin change), or simply lose its energy bit by bit as heat (internal conversion). In addition, the charge transfer state itself can decay by mechanisms other than passing the electron on to another molecule to do redox chemistry, or into an external circuit as an electric current. Charge recombination to form the ground state is always thermodynamically favorable. Reverse electron transfer to reform the excited state is also possible, although this is not favored thermodynamically. Or, the charge transfer state can decay to a triplet excited state, which may then decay to the ground state (phosphorescence). Figure 2 shows an energy state diagram of these processes and their associated rate constants. Connolly and Bolton ${ }^{11}$ have written an extensive review of organic electron donors and acceptors that have been covalently linked in order to investigate the nature of PET. Their article covers the period up to 1987 . Wasielewski ${ }^{30}$ has extended coverage up to 1992 . Donor moieties include simple phenyl derivatives, especially methoxylated phenyl systems, phthalocyanines, carotenes and porphyrins. 


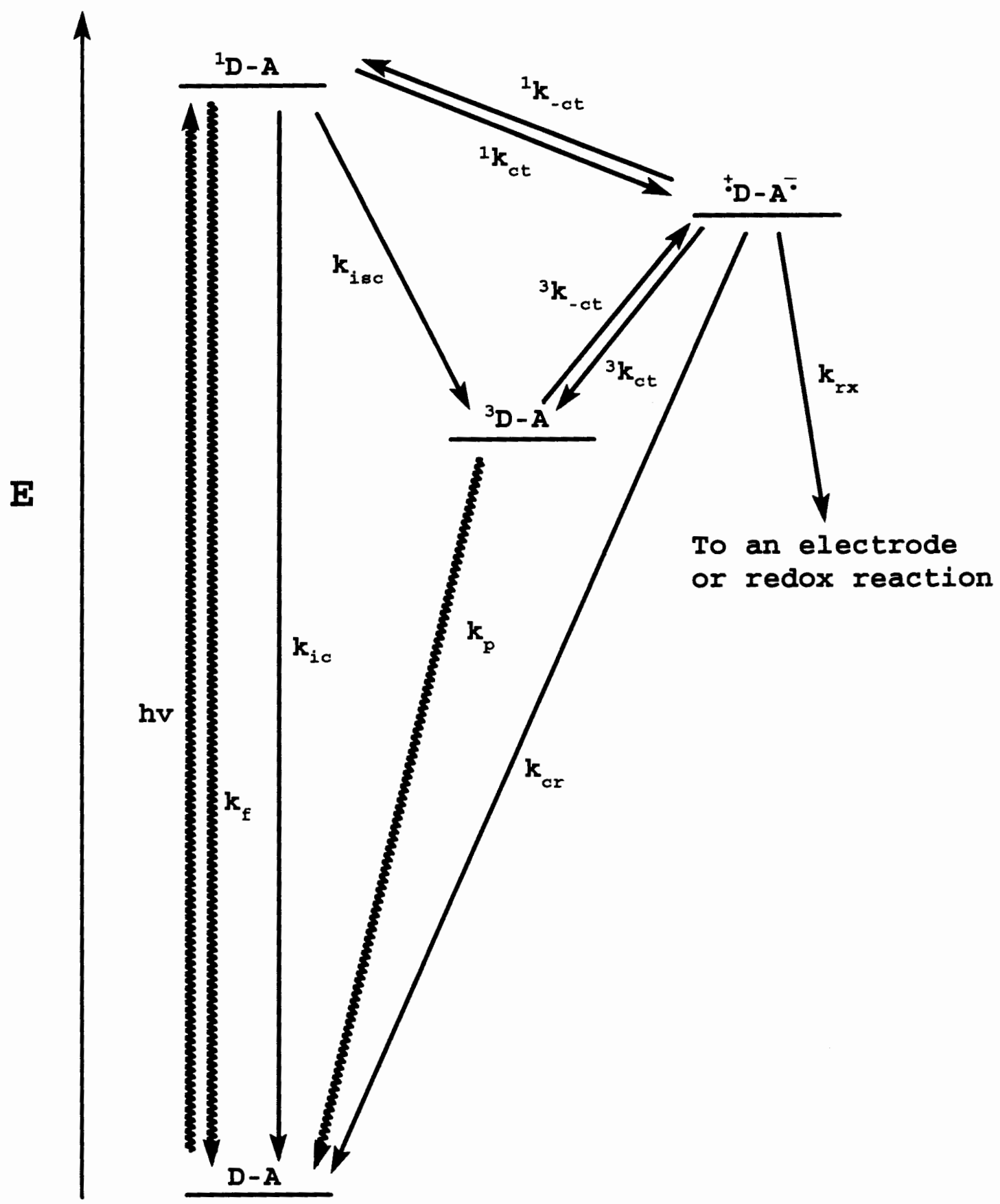

Figure 2. Energy state diagram showing various states attainable after light absorption. 
Acceptors include phenyl rings with electron-withdrawing substituents, quinones, and viologens ${ }^{11,30}$. Studies have been concerned with determining the dependence of PET rates on the free energy of reaction ${ }^{31-33}$, the effect of coordinated ligands ${ }^{34}$ and solventpolarity ${ }^{35}$, and on the donor-acceptor distance, mutual orientation ${ }^{36}$ and degree of electronic coupling ${ }^{30,37}$. A recent study was aimed at prolonging the lifetime of the charge-separated state by trapping the radical anion moiety with an intramolecular hydrogen bond ${ }^{38}$. One goal of this research is to be able to develop a system capable of high charge transfer efficiency and a low rate of charge recombination, making it ideal as an electrode sensitizer in a photovoltaic cell. With such knowledge will also come a better understanding of the fundamental photoelectronic processes in photosynthesis. To the latter end, molecular triads, tetrads, pentads and other supramolecular systems have been synthesized ${ }^{30,32,34,39-41}$ in order to mimic the long-range electron transfer process of photosynthesis, in which the photogenerated electron is moved from the reaction center chlorophyll dimer to various acceptor molecules across a membrane $35 \AA$-wide to the final quinone acceptor $^{30}$.

Electron transfer can proceed adiabatically or nonadiabatically. Adiabaticity implies that the donor and acceptor molecules are strongly electronically coupled, so that progress from the initial state to the charge-separated state remains on the lowest possible energy surface, and that once the transition state is reached, product formation occurs with $100 \%$ probability. For intramolecular electron transfer, Marcus theory gives the rate for this kind of process as: ${ }^{42}$ 


$$
k_{e t}=v_{o} \exp \left[-\frac{\left(\Delta G_{e t}+\lambda\right)^{2}}{4 \lambda k T}\right]
$$

where $v_{0}$ is the frequency of nuclear motion leading to the transition state, $\Delta G_{\mathrm{et}}$ is the free energy change of the electron transfer event (not including conformational changes leading up to the transition state and following the formation of the chargeseparated state), and $\lambda$ is the reorganization energy, i.e., the free energy change upon moving all atoms in the equilibrium precursor state (including atoms in the solvent shell) to their equilibrium positions in the product state.

Most electron transfer processes are thought to proceed nonadiabatically ${ }^{11,42}$, in which case the calculation of the rate constant is based upon a quantum mechanical treatment of the problem: ${ }^{42}$

$$
k_{e t}=\frac{4 \pi^{2}}{h}\left|H_{D A}\right|^{2}(F C)
$$

where $\mathrm{H}_{\mathrm{DA}}$ is an energy term expressing the distance dependence of the electron tunneling rate, and FC is the Franck-Condon factor, which accounts for the effects of nuclear motion on the probability of electron transfer. Classical, semiclassical, and quantum mechanical expressions for $\mathrm{FC}$ are given by Grätzel ${ }^{42}$.

The values of rate constants for intramolecular electron transfer in porphyrin donor-acceptor systems range ${ }^{31}$ from $10^{7}-10^{11} \mathrm{~s}^{-1}$. These are comparable to those for fluorescence and for energy transfer, so that charge separation is a very competitive process. 


\section{Sensitization of Semiconductors}

The mechanism and conditions for electron injection from an adsorbed dye into a metal oxide semiconductor electrode have been elucidated by Grätzel et al. ${ }^{7,13,43-47}$. Theoretical models for the current-voltage characteristics for cells based upon nanocrystalline semiconductor electrodes have been presented by Södergren, et al ${ }^{48}$. A diagram showing the operation of such a cell is given in Figure 3. The voltage output of the cell is the difference between the redox potential of the $\mathrm{A}^{+} / \mathrm{A}$ couple $(\mathrm{A}=$ electron acceptor, the reductant in the electrolyte) and the Fermi level of the semiconductor. In an n-doped semiconductor such as $\mathrm{TiO}_{2}$, the Fermi level is slightly below the conduction band. However, surface states (lattice defects at the surface) often exist at lower energies, and can both reduce the photovoltage and the photocurrent, by acting as electron traps rather than conductive states.

Light incident on the cell excites the sensitizer dye, raising the energy of an electron up to the level of the energy states in the conduction band of the semiconductor. Provided that there is adequate electronic coupling between the excited dye and the acceptor states in the conduction band, electron injection may take place. Electron injection may occur from either a singlet or triplet excited state of the dye $\mathrm{e}^{46}$. Unless there is a reducing agent present in the electrolyte, charge recombination occurs rapidly ${ }^{45}$. With single-crystal electrodes, if an external potential is applied, then the injected electron is quickly moved away from the surface by the electric field, and charge recombination is significantly reduced. However, the small size of colloidal particles prevents a substantial electric field 


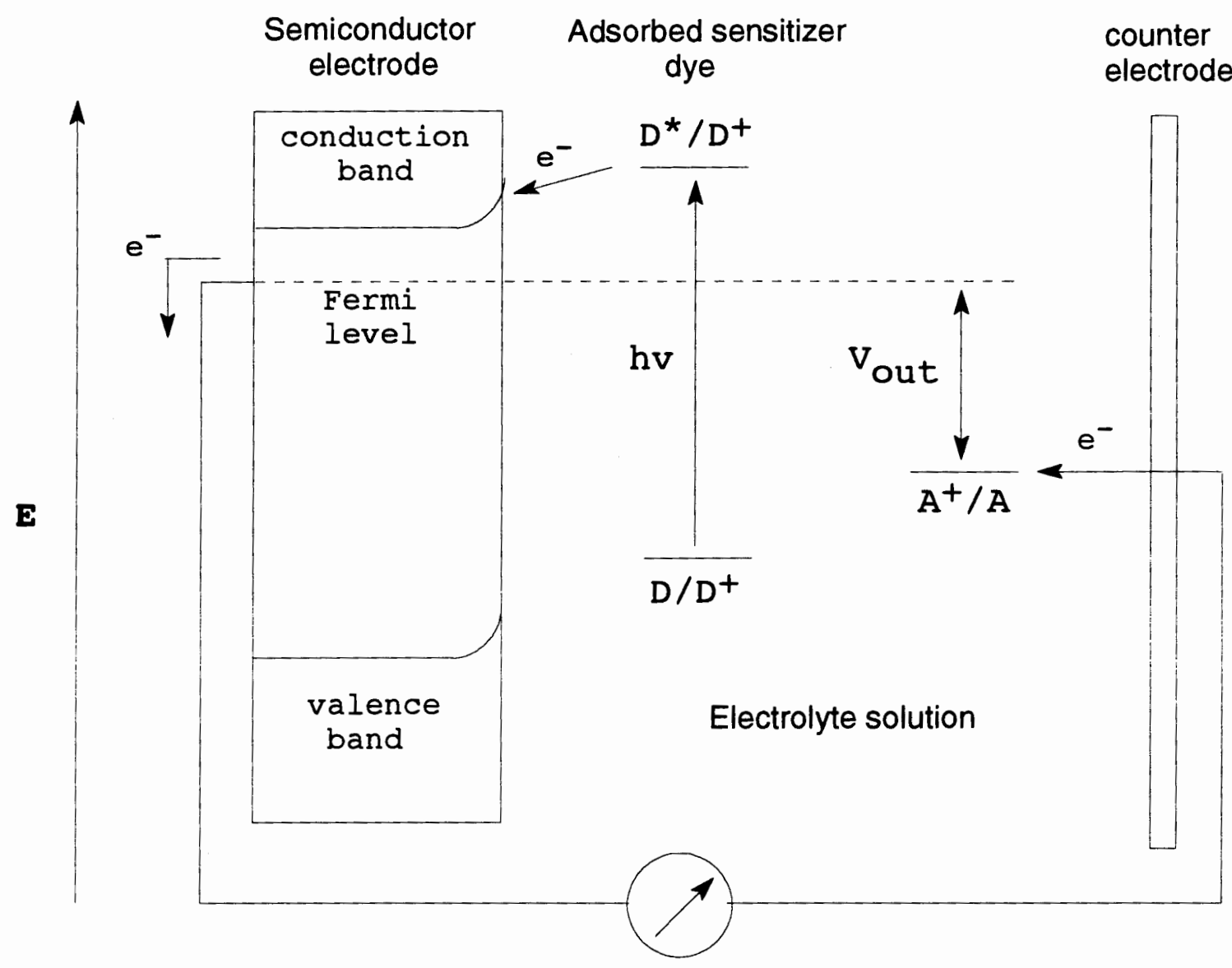

Figure 3. Schematic diagram of a photovoltaic cell utilizing a dyesensitized semiconductor electrode. $\mathrm{D}=$ electron donor (sensitizer); $\mathrm{A}$ $=$ electron acceptor (reductant).

from building up inside them when an external potential is applied ${ }^{42}$. The mechanism for charge recombination in this case appears to rely on surface states in the semiconductor particle which act as electron traps, keeping the electron in spatial proximity to the cationic dye. Adding a reducing agent prevents charge recombination by reducing the dye cation, in which case the injected electron is thought to diffuse through the semiconductor film by hopping between surface states on the colloidal particles ${ }^{46}$. Assumption of the diffusion mechanism leads to 
agreement between the theoretical current-voltage characteristics of the cell and experimental results ${ }^{48}$.

The addition of Lewis bases such as 4-t-butylpyridine causes a great increase in photovoltage, but no significant change in the photocurrent ${ }^{13}$. It was proposed that this effect is due to complexation of $\mathrm{Ti}^{+4}$ surface states, which possess high Lewis acidity. Such sites are known to act as low-energy traps for injected electrons, becoming reduced to $\mathrm{Ti}^{+3}$ centers ${ }^{46,49}$. Complexing these sites with carboxylic acids has also been shown to dramatically increase the rate of electron transfer from the conduction band of the semiconductor to acceptor species in the electrolyte ${ }^{50}$. Theoretical models for the effects of surface states on charge separation and charge transport in nanocrystalline electrodes have aided understanding of these processes ${ }^{51}$.

It seems likely that an abundance of redox active surface sites would exist in any semiconductor whose lattice is highly disordered. A recent study of its photocatalytic activity found that $\mathrm{TiO}_{2}$ consisting of both anatase and rutile structures was much more reactive than pure anatase or rutile ${ }^{52}$. Forcing the lattice to accommodate two crystalline forms in a small colloidal particle seems to create a great many sites for redox activity.

As mentioned above, in order for electron transfer to occur efficiently, there must be a thermodynamic driving force, and the dye must be covalently linked (chemisorbed) to the semiconductor surface in order to maximize orbital overlap between the dye molecule and surface atoms. The first condition is met when the redox potential for the $\mathrm{D}^{*} / \mathrm{D}^{+}$couple $(\mathrm{D}=$ electron donor, the sensitizer dye) is more 
negative than that of the acceptor states (Fermi level) in the semiconductor. The second condition has been achieved by using dyes possessing carboxylate $e^{7,13,43,46}$ or phosphonate groups ${ }^{53}$, which bind tightly to the semiconductor surface. An FTIR study of benzoic acid adsorbed onto $\mathrm{TiO}_{2}$ suggested that the nature of the linkage to the surface involved coordination of both oxygen atoms of the carboxyl group to a single surface titanium atom ${ }^{54}$. The nature of the phosphonate linkage is not known, but it appears that it is much stronger than that for carboxylates ${ }^{53}$.

Sensitizing dyes that have been utilized include porphyrins and chlorophylls, ruthenium complexes, xanthenes, phthalocyanines, 8-hydroxyquinoline complexes, coumarins and rhodamine $B^{6,7,43,44,46}$. Semiconductors that have been sensitized with such dyes include $\mathrm{TiO}_{2}, \mathrm{ZnO}, \mathrm{SrTiO}_{3}, \mathrm{MgTiO}_{3}, \mathrm{CaTiO}_{3}, \mathrm{SnS}_{2}$ and $\mathrm{SnO}_{2} 6,7,44,47,55$. Photocurrent action spectra from an electrode sensitized with the zinc derivative of one of the porphyrins ${ }^{44}$ used in this study indicated an incident photon-to-current efficiency (IPCE) of $42 \%$ at the optimum absorbance wavelength ${ }^{43}$.

\section{The Grätzel Cell}

In 1991, a Swiss research group headed by Michael Grätzel announced a lowcost, $7 \%$ efficient solar cell based on a ruthenium dye-sensitized, nanocrystalline $\mathrm{TiO}_{2}$ electrode ${ }^{55}$. The dye contained carboxylate groups which allowed it to chemisorb onto the $\mathrm{TiO}_{2}$ surface. In the following years, the basic cell design was patented $^{56}$, various modifications were made, and by 1993 , the Grätzel cell had attained an efficiency of $10 \%^{13}$. The best dye found was cis-di(thiocyanato)bis $\left(2,2^{\prime}\right.$ bipyridyl-4,4'-dicarboxylate)ruthenium(II). This compound exhibits a broad 
absorbance from about 350 to $700 \mathrm{~nm}$, and harvests incident light very efficiently. The IPCE was greater than $60 \%$ over most of the visible range of wavelengths, and reached $90 \%$ around $510 \mathrm{~nm}$. The quantum yield for charge injection from the excited dye into the conduction band of $\mathrm{TiO}_{2}$ was calculated to be greater than 99.9\%. Short-circuit photocurrent densities up to $17 \mathrm{~mA} / \mathrm{cm}^{2}$ were obtained, and open-circuit voltages up to $0.72 \mathrm{~V}$. The overall energy conversion efficiency of the cell reached a maximum of $10.4 \%$ under simulated direct AM 1.5 illumination. This was found to be very close to the theoretical maximum for cells based upon trinuclear ruthenium dyes ${ }^{57}$. Similar cells were constructed and tested by researchers in other laboratories, and found to give the same results ${ }^{5,57,58}$. The Grätzel cell was also found to be quite stable to photodegradation: each molecule could be excited at least $10^{7}$ times on average without losing its ability to inject an electron into the electrode. A cost estimate ${ }^{59}$ for producing modules based upon this type of cell fell in the range $\$ 48-\$ 64 / \mathrm{m}^{2}$. A cell operating at $8 \%$ efficiency would then produce power at $60-80 \varnothing$ per peak watt, compared to $\$ 3-\$ 8$ per peak watt for single crystal silicon cells. This cell design obviously has very great potential in future means of energy production.

It is important to carefully match the redox levels of the reductant in the electrolyte, the sensitizer, and the semiconductor in order to maximize the power output of the cell. Increasing the thermodynamic driving force between any two states may be expected to increase the rate of electron transfer and thus increase the 
photocurrent, but by the same token, the output voltage of the cell will decrease as well.

One improvement that may be suggested to this kind of cell is that it be made solid state by using a conducting polymer electrolyte ${ }^{59,60}$. The Grätzel cell contained iodide as reductant in acetonitrile. This solvent has been reported to dissolve the sealant for the cell, allowing leakage of the electrolyte ${ }^{5}$. The research described in this thesis represents the beginning of a larger project to build and test a completely solid state cell based upon a porphyrin-sensitized $\mathrm{TiO}_{2}$ electrode, in which the sensitizer is connected to the counter electrode by a conductive polymer. 


\section{CHAPTER II}

\section{RESULTS AND DISCUSSION}

\section{INTRODUCTION}

The goal of this research project was to link two commercially available porphyrins, tetrakis(4-carboxyphenyl)porphine (TCPP) and tetrakis(4aminophenyl)porphine (TAPP), via a single amide bond, to form the product shown in Figure 4. Cyclic voltammetry measurements of the redox potentials of these compounds indicate that TCPP should be a suitable electron acceptor if TAPP is the donor (see below). TCPP has four carboxyl groups, and adsorbs very tightly onto $\mathrm{TiO}_{2}$. Assuming that only a monolayer of the porphyrin adsorbs onto $\mathrm{TiO}_{2}$, values for surface coverage suggest that the molecules are not lying flat, but are oriented with the plane of the macrocycle more or less perpendicular to the surface, i.e., the porphyrin is anchored with only one or two carboxyl groups.

TCPP by itself can act as a sensitizer for a $\mathrm{TiO}_{2}$ electrode, and the resulting photocurrents and photovoltages are comparable to those reported for its zinc derivative $^{43,44,61}$. Linking TAPP onto TCPP may increase the open-circuit voltage of the cell (as compared to using iodide as a reductant, as in the Grätzel cell). Also, since TAPP can be polymerized, either with itself or with aniline, to form a conductive polymer, it is hoped that a completely solid state cell can be designed 


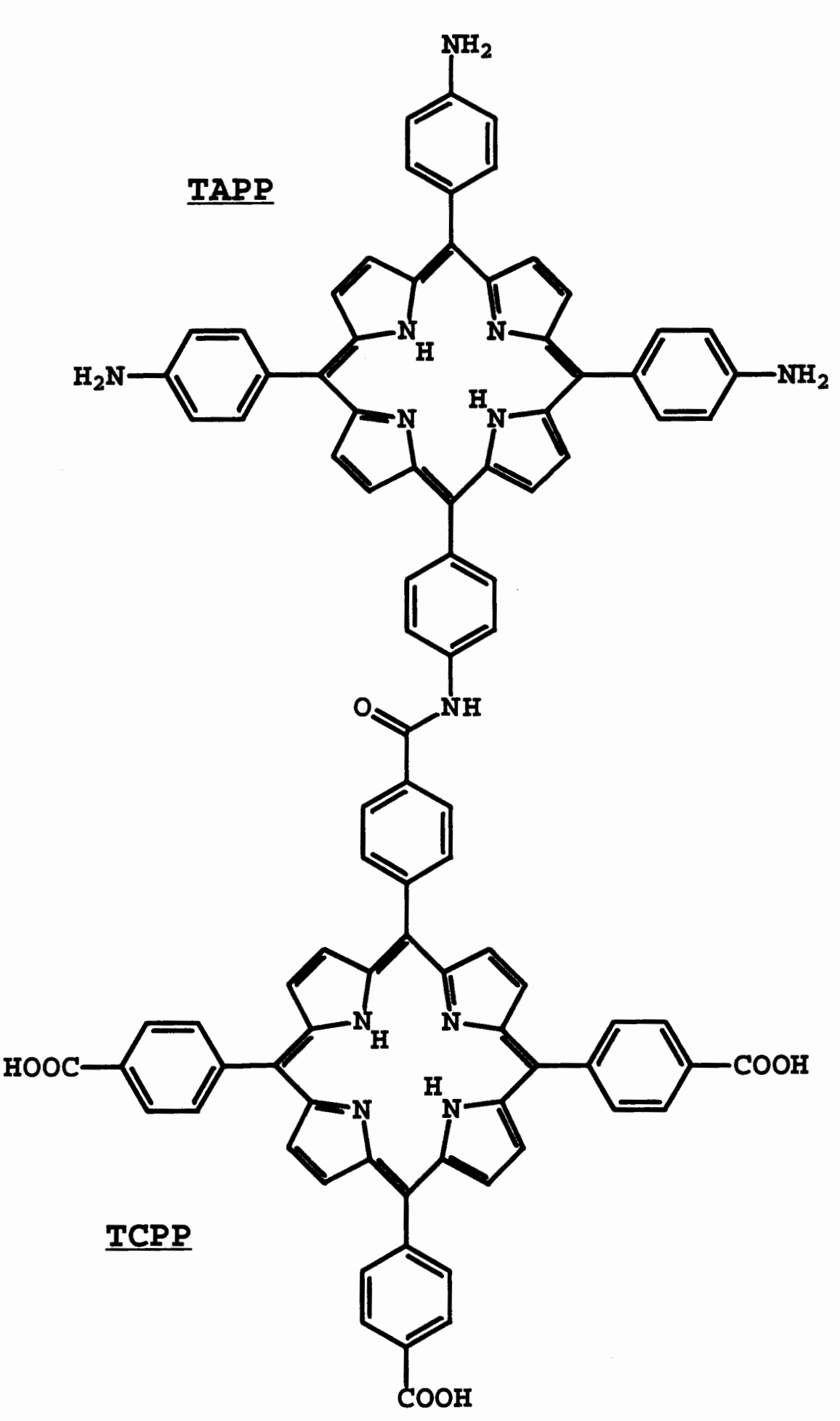

Figure 4. The synthetic goal: the TCPP-TAPP dyad. 
with such a polymer in place of the electrolyte. This would obviate the leakage problem associated with liquid electrolytes.

Figure 5 shows the relative energies of the excited states and charge separated states expected for the TCPP-TAPP dyad (recall Figure 2). The energies of the charge separated states were calculated from data acquired from cyclic voltammetry experiments performed on the monomers ${ }^{62}$ according to the following equation:

$$
E=E_{D / D^{+}}-E_{A / A^{-}}-\frac{e^{2}}{4 \pi \epsilon_{o} \epsilon I}
$$

where $\mathrm{E}_{\mathrm{D} / \mathbb{D}^{+}}$is the potential at which the donor is oxidized, $\mathrm{E}_{\mathrm{A} / \mathrm{A}^{-}}$is the potential at which the acceptor is reduced, and the last term is the Coulombic interaction energy for two ions separated by distance $r$ in a solvent with dielectric constant $\epsilon$. From cyclic voltammetric measurements in DMSO solution $(\epsilon=47)$, the values for the first two terms in Equation 10 are $+0.48 \mathrm{eV}$ and $-0.87 \mathrm{eV}$ for oxidation of TAPP and reduction of TCPP, respectively ${ }^{62}$. Using a center-to-center distance of $r=19 \AA$ for the dyad, the energy of the ${ }^{-}$TCPP-TAPP ${ }^{+}$. charge-separated state relative to the neutral ground state is calculated to be $1.33 \mathrm{eV}$. The energy of the ${ }^{+}$.TCPP-TAPP ${ }^{-}$ state is likewise calculated to be $2.28 \mathrm{eV}$, using oxidation and reduction potentials of $+1.12 \mathrm{~V}$ and $-1.18 \mathrm{~V}$, respectively. Thus electron transfer from an excited state of TAPP to TCPP is very much favored over transfer in the opposite direction.

The energies of the excited states are known from the energies of the $(0,0)$ electronic transitions. The $(0,0)$ transition occurs where the absorption and emission spectra cross, and may be approximated by taking the average of the redmost 


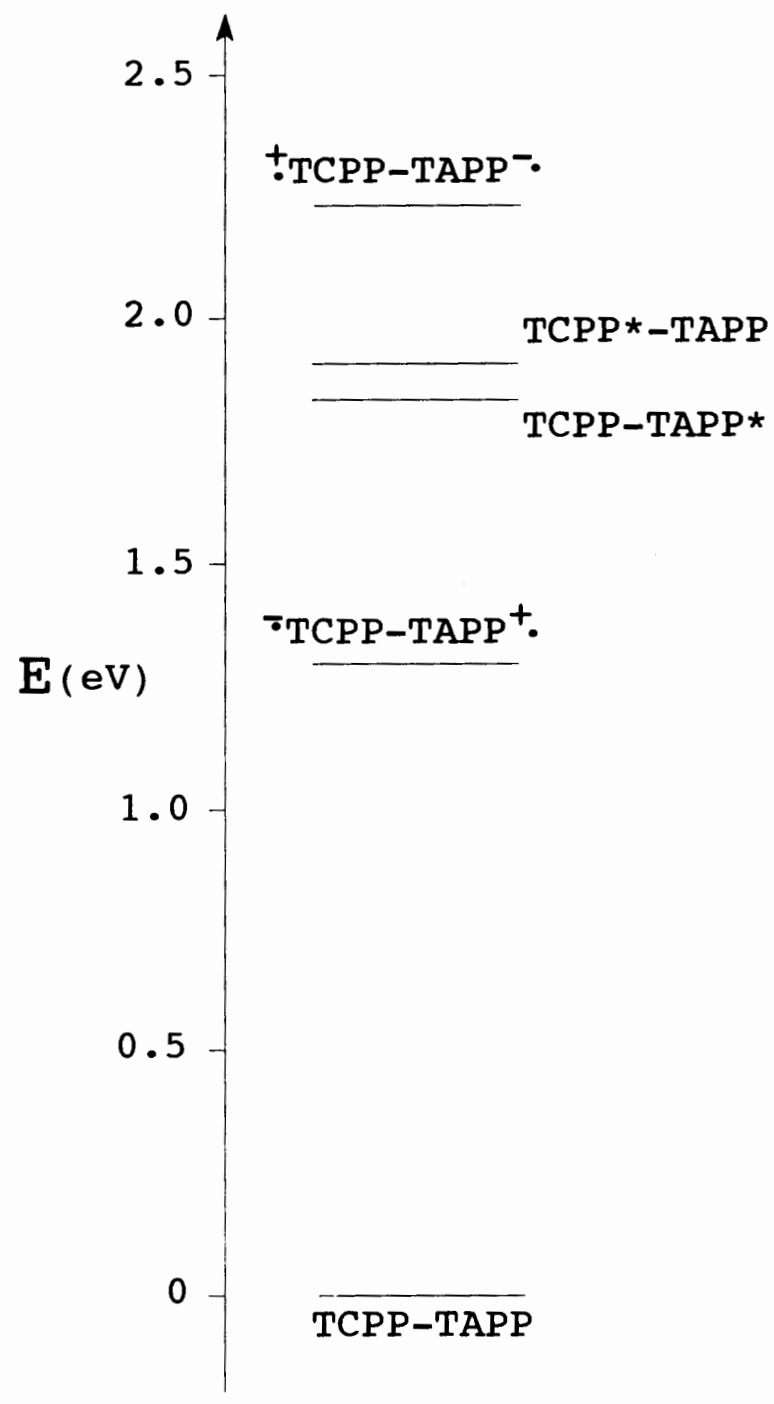

Figure 5. Energy state diagram for the TCPP-TAPP dyad.

absorption peak energy and the bluemost emission peak energy ${ }^{63}$. For TCPP and TAPP these values are $1.92 \mathrm{eV}$ and $1.84 \mathrm{eV}$, respectively. It is known that linking two different porphyrins by an amide bond does not significantly perturb either their $(0,0)$ transition energies or their redox potentials ${ }^{32,63}$, so the above calculations for the monomers are expected to hold for the dyad. One experimental imperative observed 
here is that both voltammetry and spectroscopy experiments be carried out in the same solvent, so that the environment of the porphyrins is kept constant.

It is interesting that while electron transfer is favored from TAPP to TCPP, energy transfer is favored in the opposite direction. This is not the case for other porphyrin dyads that have been reported ${ }^{31,63}$. This might introduce a problem when the dyad is adsorbed onto $\mathrm{TiO}_{2}$. Excitation of the TCPP moiety should result in electron injection into the $\mathrm{TiO}_{2}$, but the excited state could also decay by energy transfer to the attached TAPP. A new decay mode is available which is normally not thermodynamically favorable, and this may complicate the kinetics of electron injection into $\mathrm{TiO}_{2}$. The TAPP excited state could in turn transfer an electron back to TCPP, but this pathway to electron injection is indirect. Any unwanted reactions of the TAPP excited state, such as photodegradation, might be enhanced due to the increased population of TAPP excited states.

\section{ATTEMPTED SYNTHESES OF THE TCPP-TAPP DYAD}

\section{General Approach}

The structures of the various porphyrins referred to in this study, and their corresponding abbreviations, are shown in Figure 6.

The synthetic goal was to couple TAPP and TCPP via a single amide bond, as shown in Figure 4. Most amide syntheses proceed via activation of the carboxyl group with a suitable coupling agent, followed by nucleophilic attack of the amine at the carbonyl carbon. Initially, it was felt that treatment of TCPP with thionyl 


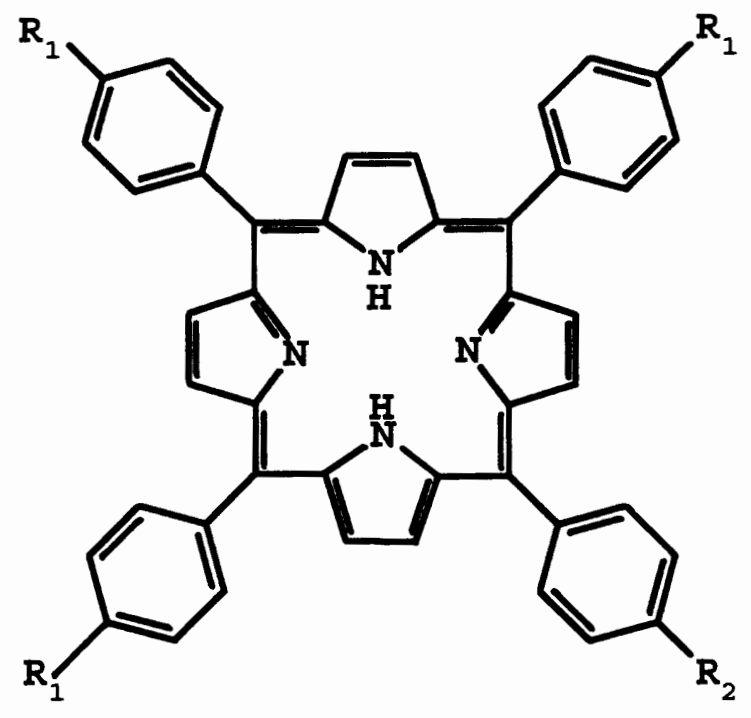

$\mathrm{R}_{1}=\mathrm{R}_{2}=\mathrm{H}$; tetraphenylporphyrin (TPP)

$\mathrm{R}_{1}=\mathrm{R}_{2}=\mathrm{COOH}$; tetrakis(4-carboxyphenyl)porphyrin (TCPP)

$\mathrm{R}_{1}=\mathrm{R}_{2}=\mathrm{COCl}$; tetraacid chloride of TCPP (TCCPP)

$\mathrm{R}_{1}=\mathrm{R}_{2}=\mathrm{COOCH}_{3}$; tetramethyl ester of TCPP (TMCPP)

$\mathrm{R}_{1}=\mathrm{COOCH}_{3} ; \mathrm{R}_{2}=\mathrm{COOH}$; trimethyl ester of TCPP (TrMCPP)

$\mathrm{R}_{1}=\mathrm{R}_{2}=\mathrm{NH}_{2}$; tetrakis(4-aminophenyl)porphyrin (TAPP)

Figure 6. Structures and abbreviations for the porphyrins referred to in this work. 
chloride might be too harsh, and that there might be problems removing the $\mathrm{HCl}$ byproduct from the reaction mixture. Although removal of $\mathrm{HCl}$ is usually not a problem since it is a gas, porphyrins are sensitive to acid because the pyrrole nitrogens inside the macrocycle become protonated. The clear indication of this is that both TCPP and TAPP become bright green, whereas their unprotonated forms give pink and dull olive-colored solutions, respectively.

Peptides are frequently constructed using carbodiimides as coupling agents to link the amino acids ${ }^{64,65}$. No acidic byproducts are involved, and the dialkylureas that are produced are removed by extraction or chromatography. The reaction paths are shown in Figure 7. A test of the viability of one of the most popular carbodiimides,
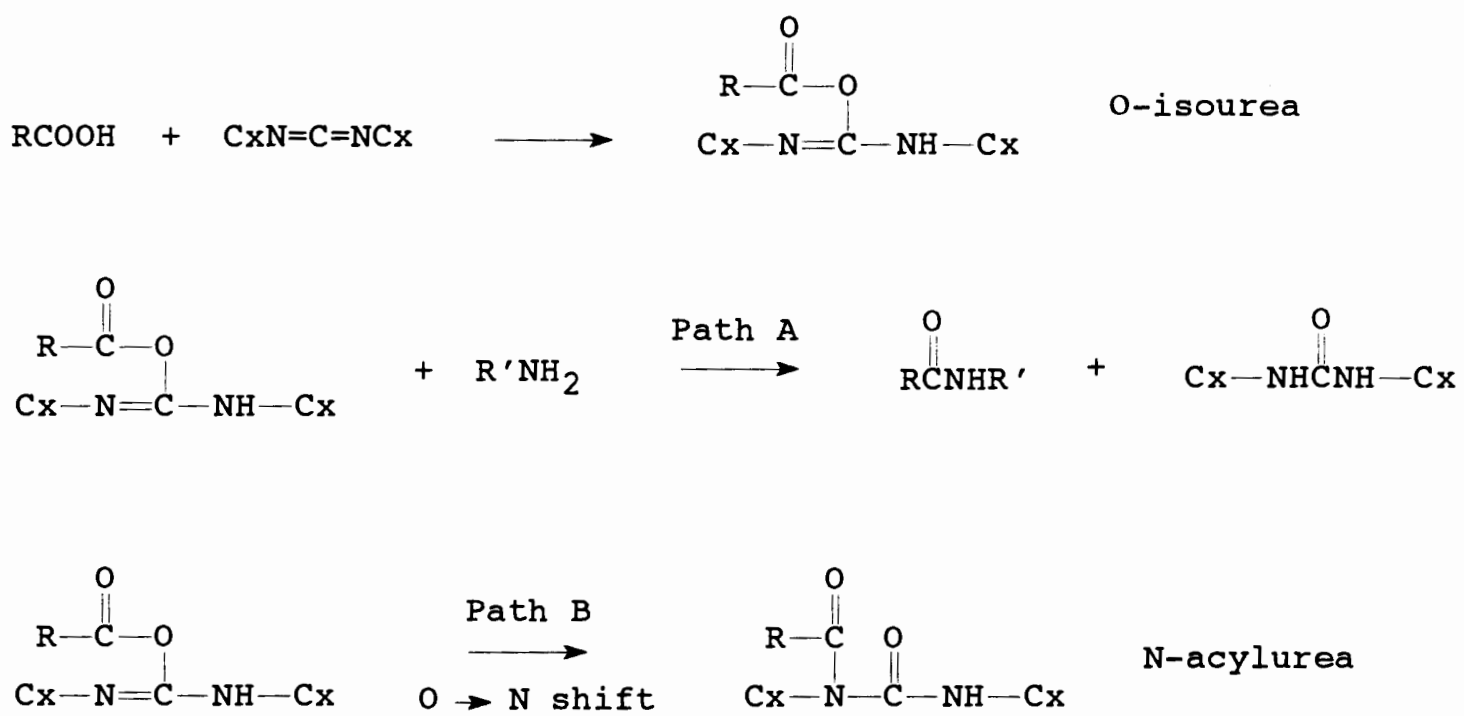

Figure 7. Reaction pathways for carbodiimide coupling namely dicyclohexylcarbodiimide (DCC), was carried out using terephthalic acid and 
aniline as models for TCPP and TAPP, respectively ${ }^{66}$. NMR indicated that this reaction was successful, because an amide proton signal for the product was observed at $10.5 \mathrm{ppm}$ (see Figure 8 and compare with the NMR spectrum of benzanilide, Figure 9). It was therefore assumed that DCC would be effective in coupling the two porphyrins. This assumption proved to be a serious error, because more than a year was spent using this reagent in various attempts to synthesize the product, until the conclusion that the carbodiimide method was ineffective proved inescapable. The reason seems to be due to the poor nucleophilicity of TAPP. TAPP is known to be a weaker base than aniline $\left(\mathrm{pK}_{\mathrm{a}}=4.2\right)$ by the fact that it becomes soluble only at $\mathrm{pH} 3$ or $\mathrm{so}^{67}$. To the extent that nucleophilicity correlates with basicity (particularly valid for a series of compounds with the same functional group $\left.{ }^{68}\right)$, it is assumed that the rate of attack by TAPP on the activated TCPP is too slow to compete with the rearrangement of activated acids to the unreactive $\mathrm{N}$-acylureas (path $\mathrm{B}$ in Figure 7).

No evidence of product formation was observed in later reactions using either DCC or diisopropylcarbodiimide (DIC). A simple test was finally carried out by dissolving TCPP, TAPP and DIC in a solvent and allowing the porphyrins to react as much as possible. The NMR spectrum of the product mixture (Figure 10) shows no change in peak positions for TAPP (Figure 11), but the phenyl proton resonances for TCPP (Figure 12) are shifted significantly. Furthermore, there are two resonances for the methyl hydrogens of the carbodiimide with approximately equal integrals at 1.01 and $1.43 \mathrm{ppm}$. The chemical shift of the methyl hydrogens in DIC and in diisopropylthiourea are identical ${ }^{69}$ (both at $1.22 \mathrm{ppm}$ in $\mathrm{CDCl}_{3}$; no standard spectrum 
17 peaks found in NMR2.DB

peak ppm freq amp

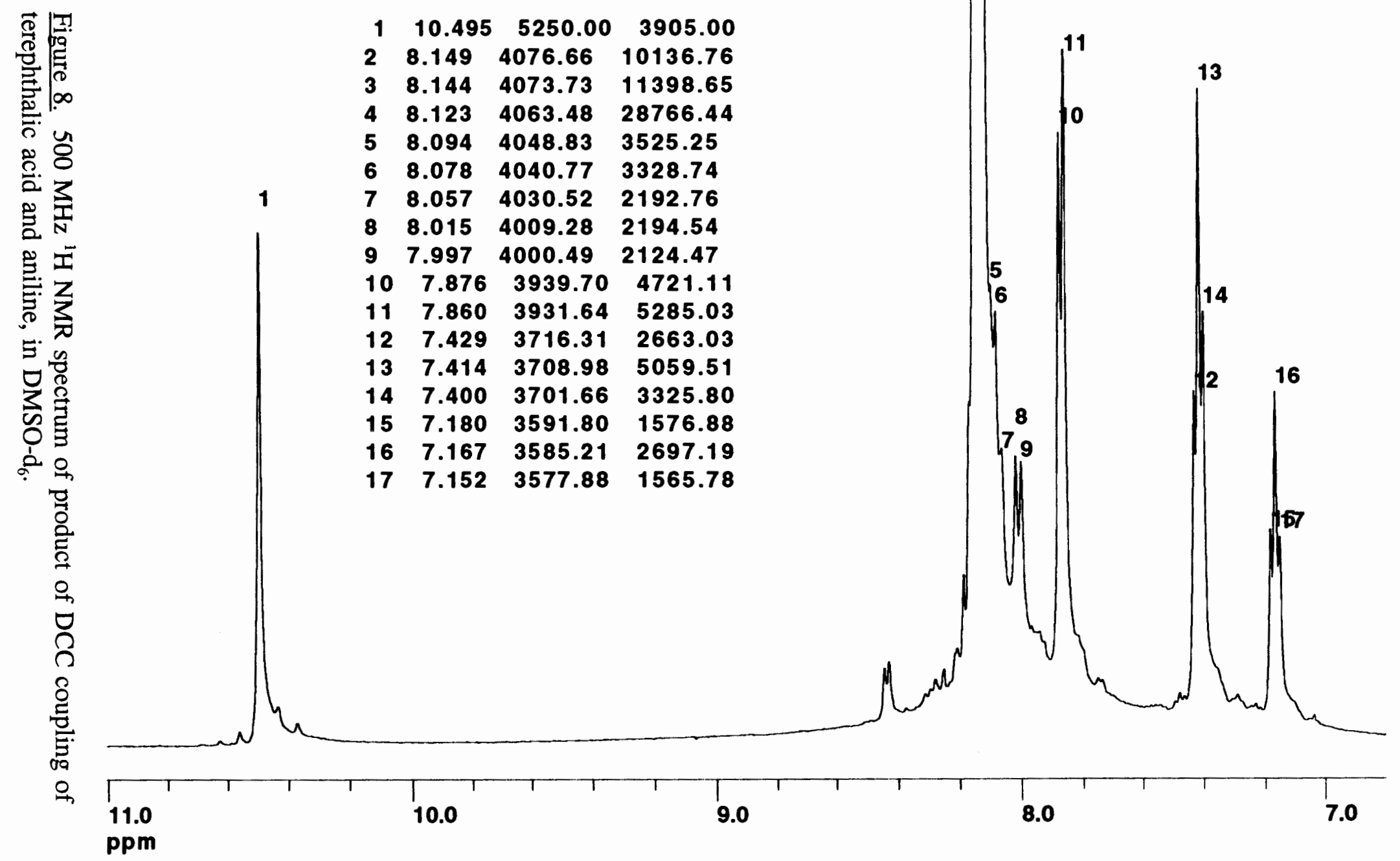




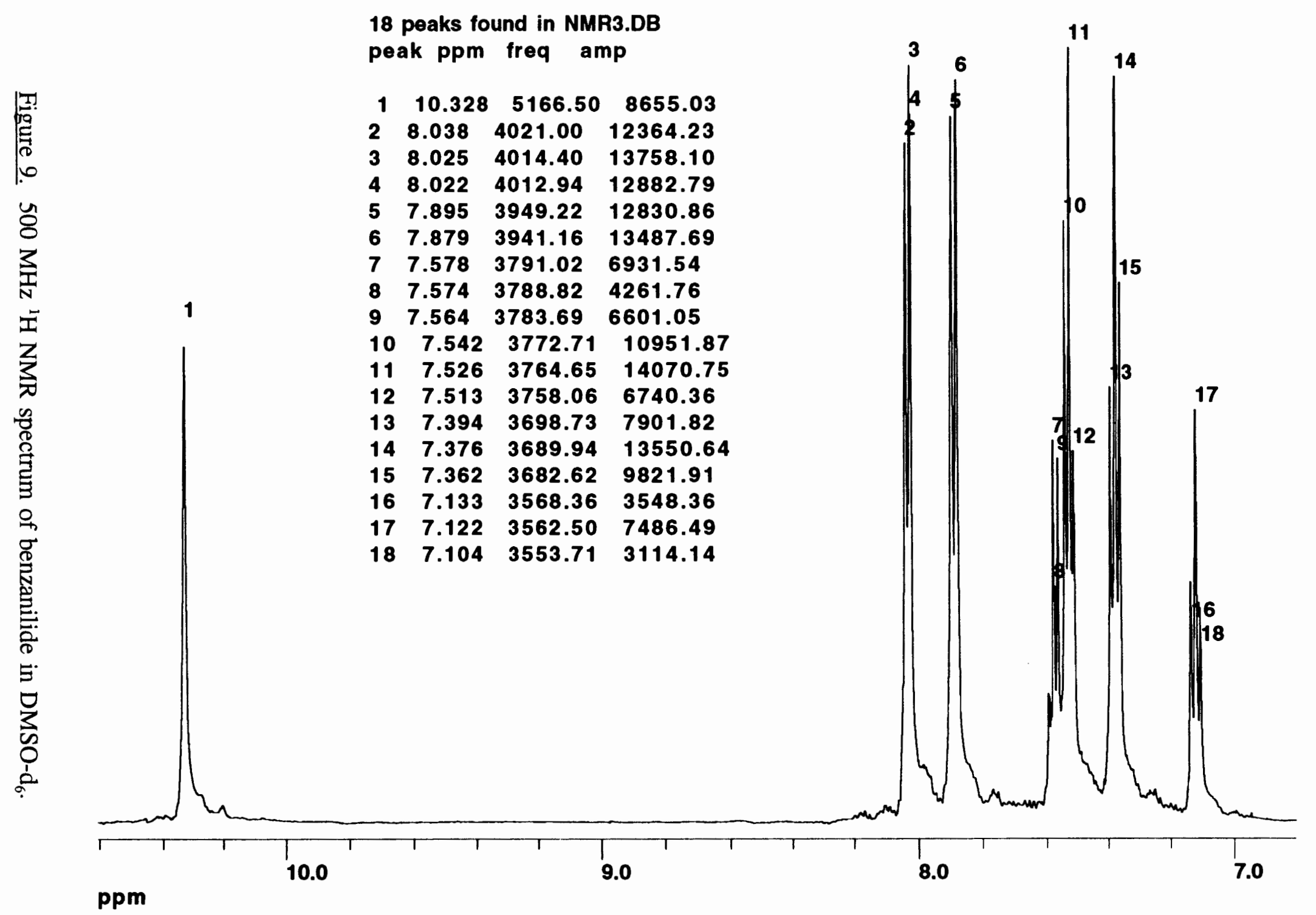


$56 \cdot 2-$

\2:2-

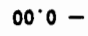

98.0

$10 \%$
$20 \%$

दा'

$02: 1$

เ2.

OE 1

टE. 1

다. 1

$n \cdot 1$

IS'

id.

TE' $E$

IS'E

$E L \cdot E$

$\checkmark L E$

$9 L \cdot E$

SL' $\quad$ Q

$90 \div$
$0<\div$

6S's -

00.2

$20^{\circ} \mathrm{L}$

LF $L J$

$r 0^{\circ} L$

92.

$26 . \angle$

16.2

S2.

$\angle 2 \cdot 8\}$

IE. 8

$10.8 \%$

$88^{\circ}$
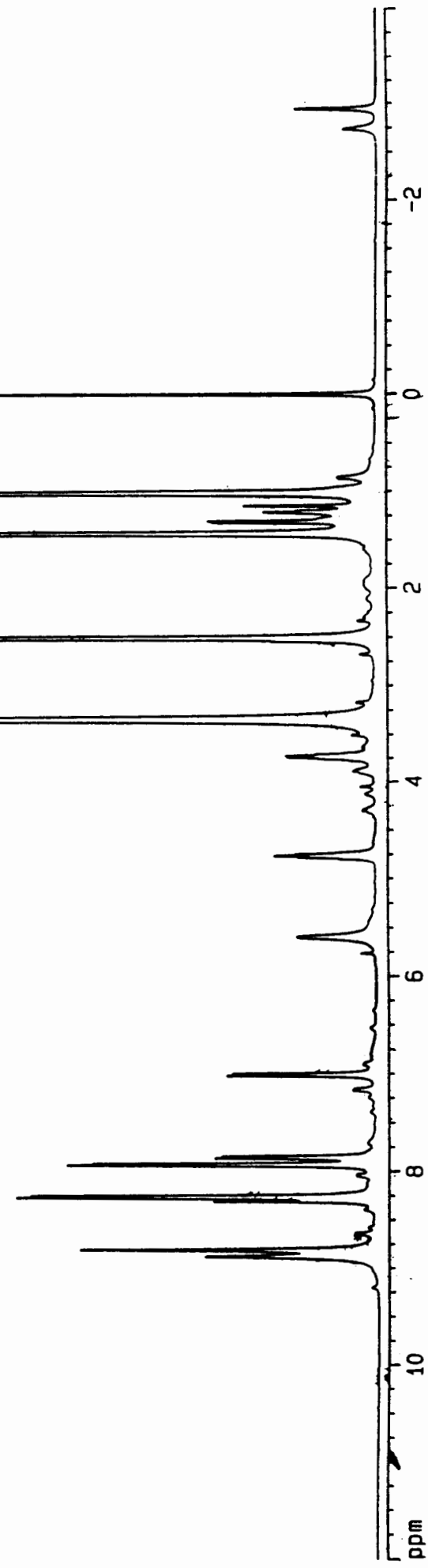

Figure 10. $400 \mathrm{MHz}{ }^{1} \mathrm{H}$ NMR spectrum of the product mixture from DIC coupling of TCPP and TAPP. 


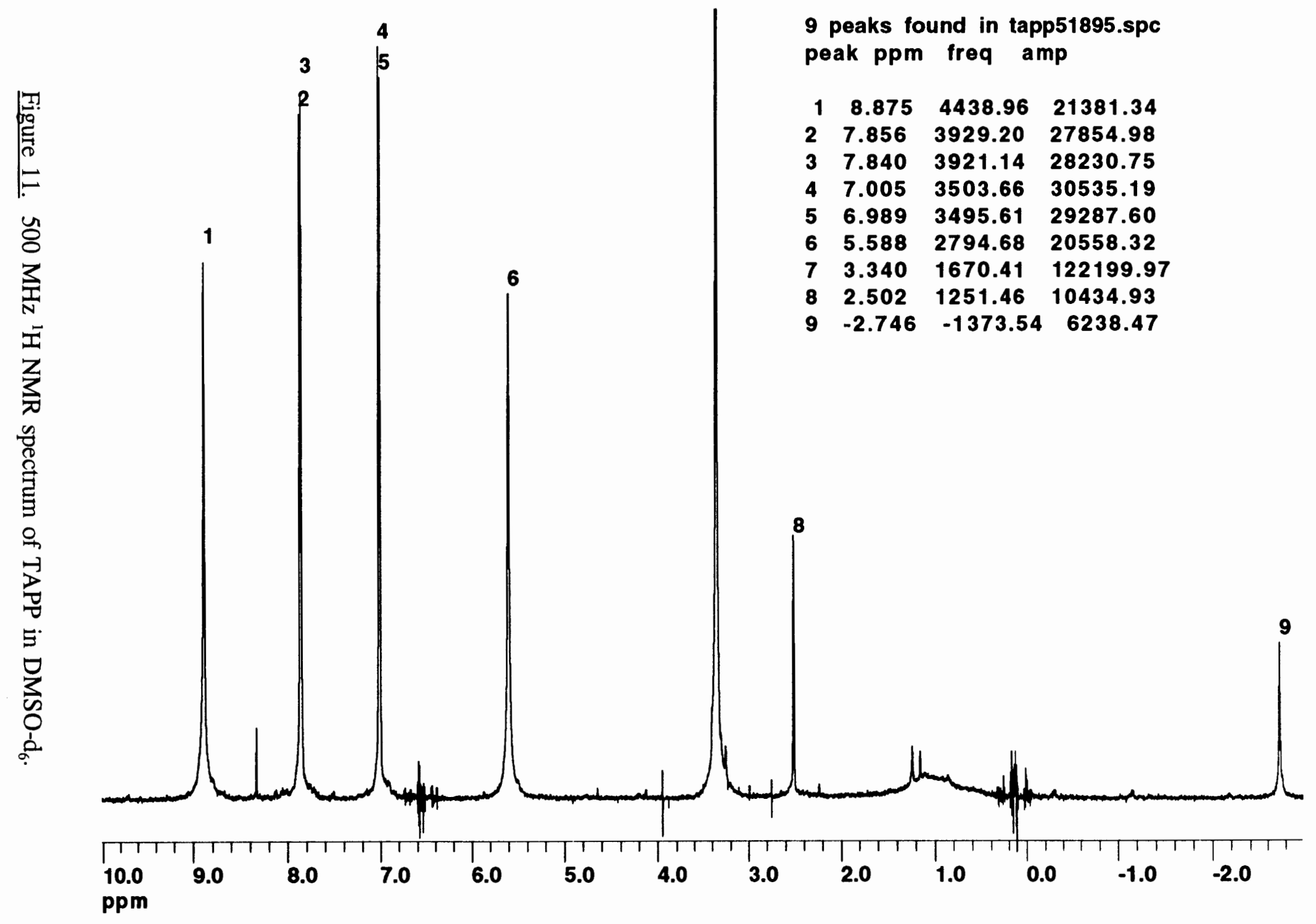




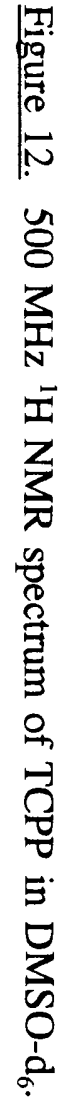

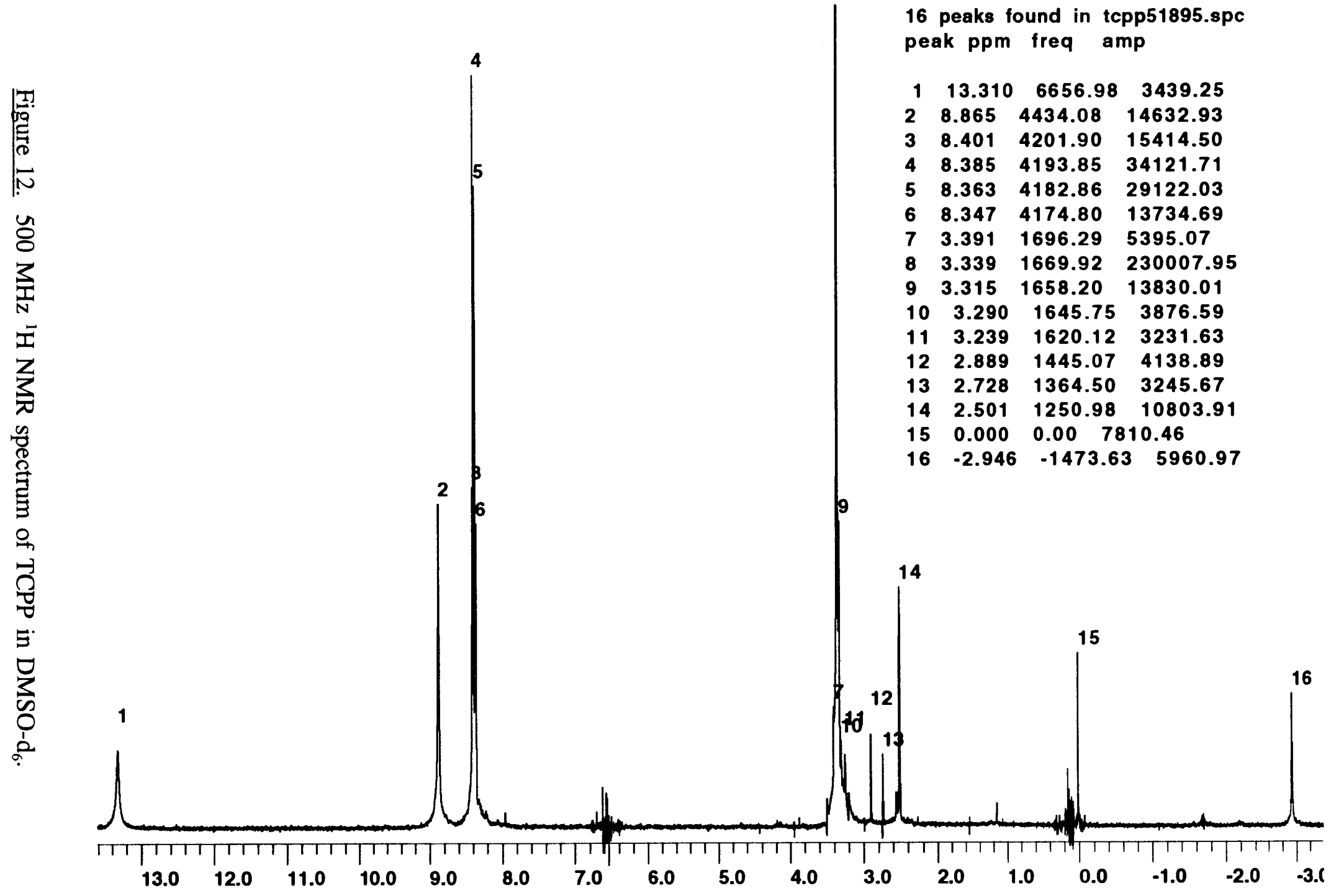
ppm 
of diisopropylurea could be found). The spectrum is thus consistent with the $\mathrm{N}$ acylurea of TCPP, in which the isopropyl groups are no longer equivalent because the molecule is not symmetrically substituted. Further evidence of this is the fact that the integral of either one of the methyl peaks is close to three times that of one of the phenyl proton resonances for TCPP, consistent with activation of all four carboxyl groups of TCPP ( 8 ortho phenyl protons : 24 methyl protons). Since TAPP was present (in slight excess) during reaction of TCPP with DIC, it appears that the process of rearrangement of the $\mathrm{O}$-isourea to the $\mathrm{N}$-acylurea was faster than nucleophilic attack by TAPP.

To test the effectiveness of thionyl chloride as a coupling reagent, it was used to convert TCPP to the tetraacid chloride (TCCPP), which was then reacted with TAPP. The products were polymeric, to judge from the fact that they were almost insoluble in all solvents. It was hoped that a resonance for an amide hydrogen would be observed in the NMR spectrum, but not enough material could be dissolved.

\section{Synthesis using a Merrifield resin}

Until it was learned that carbodiimides were ineffective coupling reagents for TCPP and TAPP, a major problem in synthesizing the TCPP-TAPP dyad was assumed to be formation of higher molecular weight products. One way of dealing with this problem was to anchor TCPP onto a very large substrate, thereby blocking one carboxyl group and perhaps effectively hindering the two nearest carboxyl groups, leaving only the one opposite exposed for activation and reaction with TAPP. 
As it turned out, even if coupling with a carbodiimide had been successful, the overall reaction would have probably failed, for reasons given below.

The first "anchor" employed was a Merrifield resin. Such resins have been used for many years in synthesizing peptides ${ }^{70,71}$. The resin consists of a polystyrene backbone substituted in places with some kind of active site to which a carboxyl group of an $\mathrm{N}$-protected amino acid can be attached. After deprotection, a second $\mathrm{N}$ protected amino acid is added along with a coupling reagent, often a carbodiimide. Reaction can only occur between the free amine group on the resin-anchored amino acid and the activated carboxyl group of the added amino acid, and thus the desired peptide bond is formed. The process is repeated until the peptide is complete. A cleavage reagent is then added to remove the peptide from the resin.

The resin employed here contained $0.66 \mathrm{mmol} / \mathrm{g}$ of accessible 2-chlorotrityl chloride active sites. Trityl esters are easily cleaved in dilute acid, due to the stability of the trityl (triphenylmethyl) cation ${ }^{72}$. In this case, no protection of amine groups was necessary, since neither porphyrin is an amino acid. TCPP could be attached to the resin, activated with DIC, reacted with TAPP to form the dyad, and then cleaved from the resin. The desired reaction scheme is shown in Figure 13.

A surprising problem was that very little TCPP could be removed from the resin. This was true even if no TAPP or DIC was added. Only $2 \%$ of the TCPP that attached to the resin could be recovered. It was noticed that addition of acid to the resin was slow to turn the adsorbed porphyrin green, and that addition of base was slow (many hours) to restore the pink color. This suggests that the cleavage 


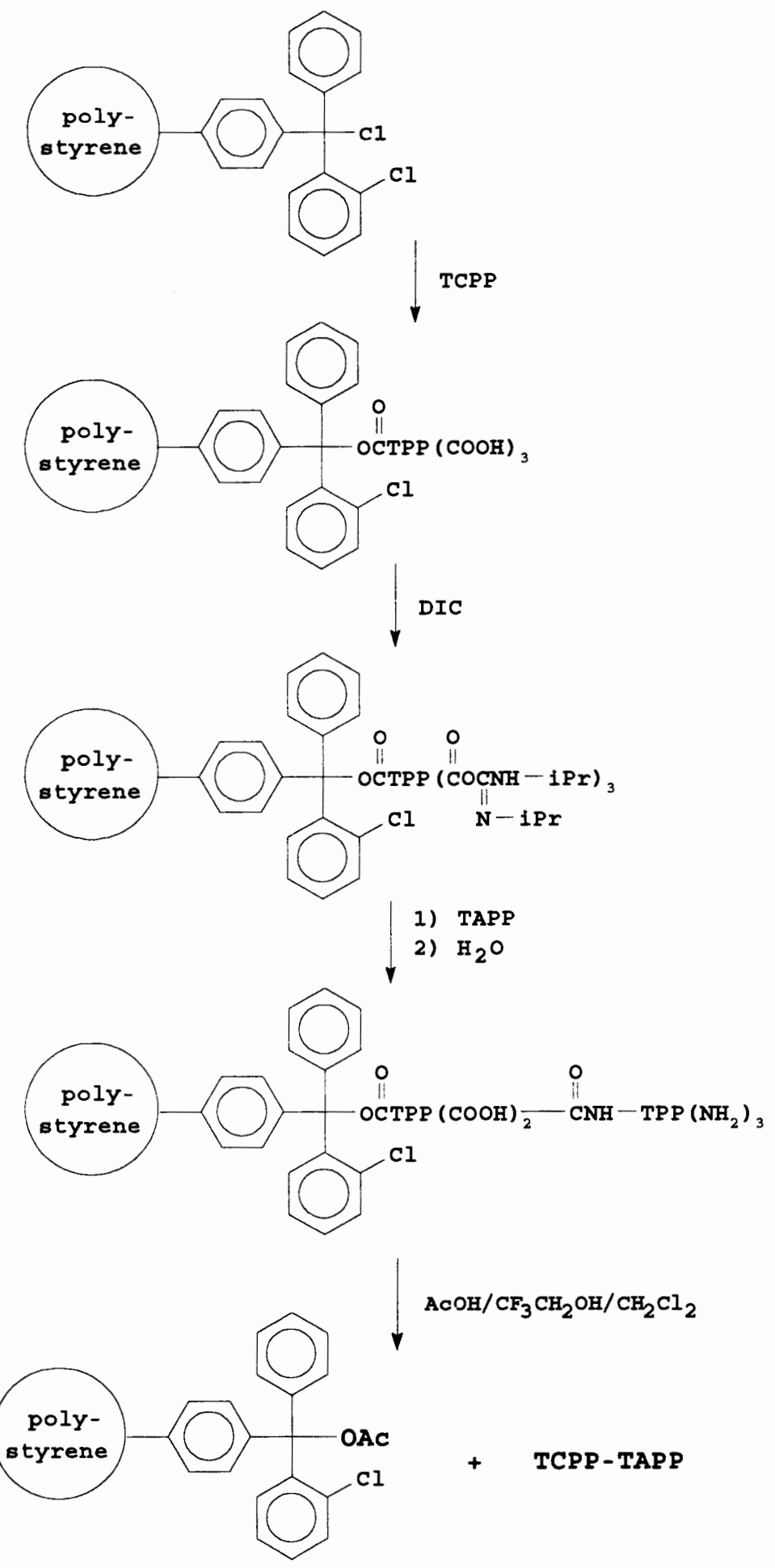

Figure 13. Synthetic scheme for the TCPP-TAPP dyad using a Merrifield resin. 
solution was slow in penetrating the resin, and perhaps also ineffective in dissolving the porphyrin if it was actually cleaved. It is possible that the porphyrin has unusually strong interactions with the resin itself, such as $\pi-\pi$ interactions with the phenyl rings of the styrene chain.

\section{Synthesis on $\mathrm{TiO}_{2}$ colloids}

Since TCPP adsorbs so well onto $\mathrm{TiO}_{2}$, colloids of this material were thought to be an ideal substrate for the coupling reaction. TCPP could be easily adsorbed from solution onto the colloids, activated with DIC, reacted with TAPP, and then cleaved using $2 \mathrm{M} \mathrm{NaOH}$ solution. Use of DIC was of course doomed to failure for the reason given above, but thionyl chloride was also employed. This seemed to react with the surface of $\mathrm{TiO}_{2}$ itself, possibly converting surface $\mathrm{Ti}-\mathrm{OH}$ groups to Ti$\mathrm{Cl}$ groups. Very little material could be removed from the colloids after this treatment.

Addition of TAPP to $\mathrm{TiO}_{2}$ that had been treated directly with $\mathrm{SOCl}_{2}$ resulted in attachment of the porphyrin to the surface, but very little could be removed using methanol, aqueous $\mathrm{HCl}$, or pyridine. TAPP can also sensitize a $\mathrm{TiO}_{2}$ electrode, but it adsorbs only weakly, and the rerring photocurrent and photovoltage are low ${ }^{61}$. This method thus represents a means of attaching TAPP more firmly to $\mathrm{TiO}_{2}$. If its electron injection capability is much improved, there would be no need for TCPP. This possibility remains to be investigated.

Conversion of $\mathrm{TiO}_{2}$-adsorbed TCPP to the acid chloride was also attempted using triphenylphosphine $/ \mathrm{CCl}_{4}$. This reaction is thought to proceed as follows: ${ }^{73}$ 


$$
\begin{aligned}
& \phi_{3} \mathrm{P}+\mathrm{CCl}_{4}->\left[\phi_{3} \mathrm{PCl}^{+} \mathrm{CCl}_{3}^{-}\right. \\
& {\left[\phi_{3} \mathrm{PCl}\right]^{+} \mathrm{CCl}_{3}^{-}+\mathrm{RCOOH}->\mathrm{RC}(\mathrm{O}) \mathrm{Cl}+\mathrm{CHCl}_{3}+\phi_{3} \mathrm{P}=\mathrm{O}}
\end{aligned}
$$

This method also converts alcohols to alkyl chlorides, and might also be expected to react with surface hydroxyl groups on $\mathrm{TiO}_{2}$. An unfortunate choice of solvent was made in this reaction, 1,2-dichloroethane, which appeared to be degraded somewhat by the $\mathrm{TiO}_{2}$, with the formation of $\mathrm{HCl}$. The porphyrin could not easily be removed from the $\mathrm{TiO}_{2}$ using the usual cleavage reagent, $2 \mathrm{M} \mathrm{NaOH}$. Although TAPP is not soluble in aqueous base, it was expected that cleavage of the TCPP- $\mathrm{TiO}_{2}$ linkage should still have occurred, leaving three ionized carboxyl groups on the dyad, which should render it soluble. Otherwise it should have simply precipitated. Addition of DMSO to the basic solution resulted in some desorption of porphyrin, turning it bright green due to deprotonation of the pyrrole nitrogens. However, fluorescence spectra of the neutralized solution gave no indication of the presence of TAPP.

It would be well worthwhile to test this coupling procedure on TCPP and TAPP in the absence of $\mathrm{TiO}_{2}$. A similar procedure using $\mathrm{CBrCl}_{3}$ is a simple onestep process in which the reactants are all refluxed together in a suitable solvent ${ }^{74}$. There is no reason why the same procedure could not be used with $\mathrm{CCl}_{4}$.

\section{Synthesis from methylated TCPP}

The final strategy for synthesizing the TCPP-TAPP dyad was to block three of the carboxyl groups of TCPP by methylation, thus limiting reactivity to the one remaining site, and facilitating chromatography of the product. This was to be 
followed by activation of the remaining carboxyl group using either thionyl chloride or $\phi_{3} \mathrm{P} / \mathrm{CCl}_{4}$, and addition of this solution to another containing TAPP. This method should eventually be successful, but there was not enough time in this study to work out proper conditions. The overall experiment was as follows:

$$
\begin{aligned}
& \mathrm{SOCl}_{2} \\
& \text { TCPP ---.--> TCCPP } \\
& \mathrm{TCCPP}+2 \mathrm{MeOH}--->\operatorname{TPP}(\mathrm{COOMe})_{2}(\mathrm{COOH})-\mathrm{COCl} \\
& \mathrm{TPP}(\mathrm{COOMe})_{2}(\mathrm{COOH})-\mathrm{COCl}+\mathrm{TAPP}(\mathrm{xs})--.-> \\
& \mathrm{TPP}(\mathrm{COOMe})_{2}(\mathrm{COOH})-\mathrm{CONH}-\mathrm{TPP}\left(\mathrm{NH}_{2}\right)_{3}
\end{aligned}
$$

TPP $(\mathrm{COOMe})_{2}(\mathrm{COOH})-\mathrm{CONH}-\mathrm{TPP}\left(\mathrm{NH}_{2}\right)_{3}+\mathrm{NaI}(\mathrm{xs})+\mathrm{NaCN}(\mathrm{xs})-->$

$$
\mathrm{TPP}(\mathrm{COONa})_{3}-\mathrm{CONH}-\mathrm{TPP}\left(\mathrm{NH}_{2}\right)_{3}+\mathrm{MeCN}+\mathrm{NaI}
$$

TCPP was refluxed in thionyl chloride to convert all carboxyl groups to acid chlorides. It was assumed that one carboxyl group would not be activated (on average), or that at least one acid chloride group would hydrolyze at some point during the reaction, so only two equivalents of methanol were added to block carboxyl groups. An excess of TAPP was then added to ensure complete reaction with any remaining acid chloride groups. A series of Soxhlet extractions was carried out on the product mixture, followed by silica gel chromatography, and extraction of the silica gel. Much of the material remained permanently bonded to the silica gel, and was lost (see the Chromatography section below), and what was finally isolated was not pure. However, a MALDI (matrix-assisted laser desorption/ionization) mass spectrum (Figure 14) indicated the presence of at least one form of the dyad in this 


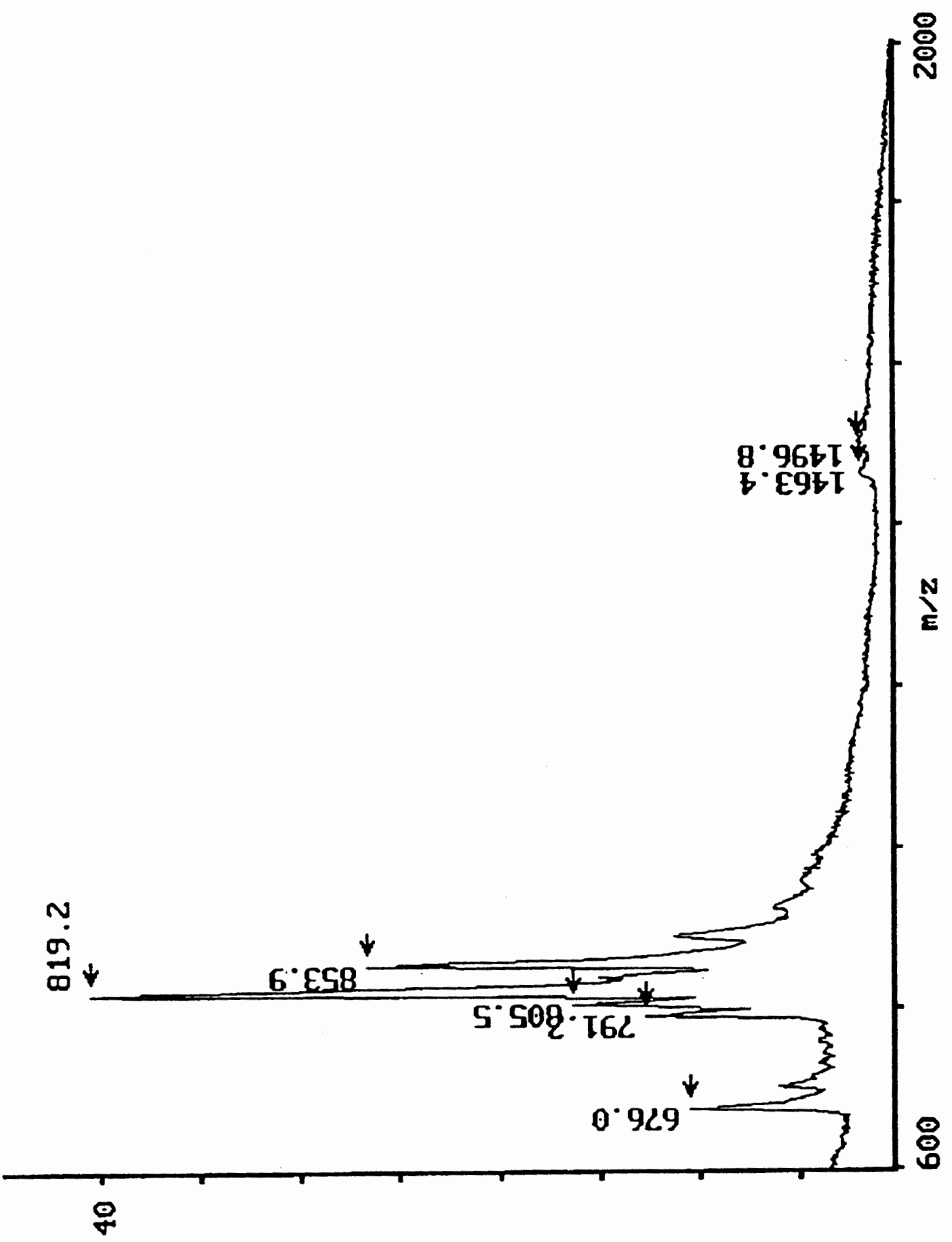

Figure 14. MALDI mass spectrum of the products of reaction of TAPP with a TCPP acid chloride. 
mixture, at a molecular weight of 1463. The dyad has a molecular weight of 1447 , and its esters have molecular weights of 1461,1473 and 1485 , respectively. Also present were TAPP, TCPP, the mono-, di-, and tetramethyl esters of TCPP, and the TCPP-TAPP 2 triad (the triad showed up more clearly in another spectrum). Clearly the attempts at purifying the mixture had been unsuccessful. The mixture was treated with $\mathrm{NaCN} / \mathrm{NaI}$ in hot DMF to remove methyl groups from the esters, and then extracted with aqueous base to isolate all ionized molecules. It was expected that the dyad would be soluble with three ionic charges on it (though it would probably micellize) and thus could be extracted from the mixture into aqueous solution. This procedure failed, possibly because demethylation was not complete. Treatment of methyl benzoate with $\mathrm{CN}^{-} / \mathrm{I}^{-}$in DMF at $120^{\circ}$ is known to cleave the ester linkage overnight ${ }^{75}$, but it was found that reflux temperature $\left(\approx 155^{\circ}\right)$ was required for demethylating the tetramethyl ester of TCPP (TMCPP, see below).

It was then decided to repeat the above reaction, but isolate the trimethyl ester of TCPP first, activate it, and add the resulting solution to another solution of TAPP to form the dyad. Methyl groups would be left on, and the product would be isolated using column chromatography:

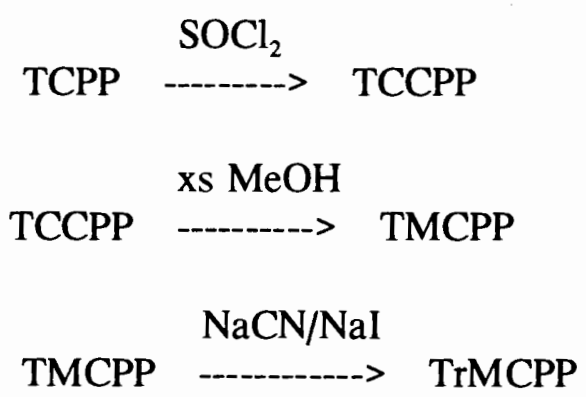




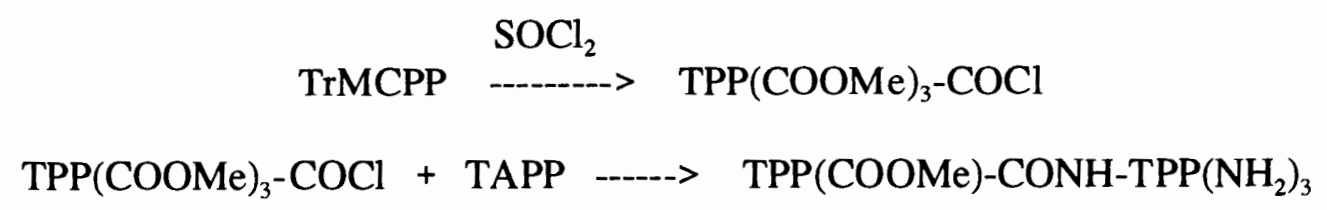

Synthetically, it is easier to form the trimethyl ester (TrMCPP) by demethylating the tetramethyl ester (TMCPP) than by trying to add three equivalents of methanol to the tetraacid chloride of TCPP (TCCPP). The reasons are: 1) it is too difficult to keep the reaction mixture free of water; 2) three equivalents of methanol amount to only a few microliters, and it is difficult to measure this accurately; 3 ) the formation of TCCPP is probably not quantitative, so three equivalents of methanol is not appropriate anyway.

TMCPP was synthesized by refluxing TCPP in thionyl chloride and then adding excess methanol. In fact, TLC showed that the product mixture contained all possible ester products, as well as TCPP. Chromatographic conditions were worked out for isolating both TrMCPP and TMCPP (see below). The TMCPP was partially hydrolyzed, and the TrMCPP was collected by chromatography. The unreacted TMCPP was also collected and hydrolyzed again, and the process repeated until enough TrMCPP had been accumulated for the next stage of the process. The hydrolysis proved to be surprisingly difficult, in keeping with the intractable nature of these compounds. Refluxing the ester in $\mathrm{THF} / \mathrm{OH}^{-}$for more than 15 hours had no effect, nor did adding cyanide or iodide. What finally worked was to reflux the ester in the presence of both cyanide and iodide in DMF.

Treatment of the TrMCPP with thionyl chloride was probably successful in 
activating the carboxyl group, but not all of the $\mathrm{HCl}$ could be removed from the product, even after leaving it in a vacuum oven overnight at $80^{\circ}$. No reaction with TAPP occurred, and the TrMCPP was recovered via chromatography. The reaction was carried out in the presence of an excess of pyridine, so any remaining $\mathrm{HCl}$ should not have caused a problem. The reaction was allowed to run for two hours, but perhaps this was not long enough, given the poor nucleophilicity of TAPP.

\section{SPECTROSCOPY}

\section{Ultraviolet/Visible Spectroscopy}

Absorption and fluorescence spectra of TCPP and TAPP in various solvents are presented in the Appendix. The two porphyrins can easily be recognized by their absorption spectra. The most intense peak is called the Soret band, and represents the $S_{0}->S_{2}$ transition. The four weaker $S_{0}->S_{1}$ transitions are designated as $Q_{y(1,0)}, Q_{y(0,0)}, Q_{x(1,0)}$ and $Q_{x(0,0)}$, respectively. The subscripts refer to the $x$ and $y$ axes of symmetry present in the free base form of the porphyrins, and to the vibrational levels of the excited and ground states, respectively. The insertion of a metal atom into the cavity of the porphyrin macrocycle, or protonation or deprotonation of the pyrrole nitrogens increases the symmetry of the molecule. The $\mathrm{x}$ and $\mathrm{y}$ axes become equivalent, and only two $Q$ bands are then observed, $Q_{(0,0)}$ and $Q_{(1,0)}$.

The integrated absorbance between $330-800 \mathrm{~nm}$ for equimolar solutions of the monomers in DMSO differs by only $2 \%$, but the Soret peak of TCPP is much more intense than that of TAPP. The absorbance spectrum of a 1:1 solution of the two 


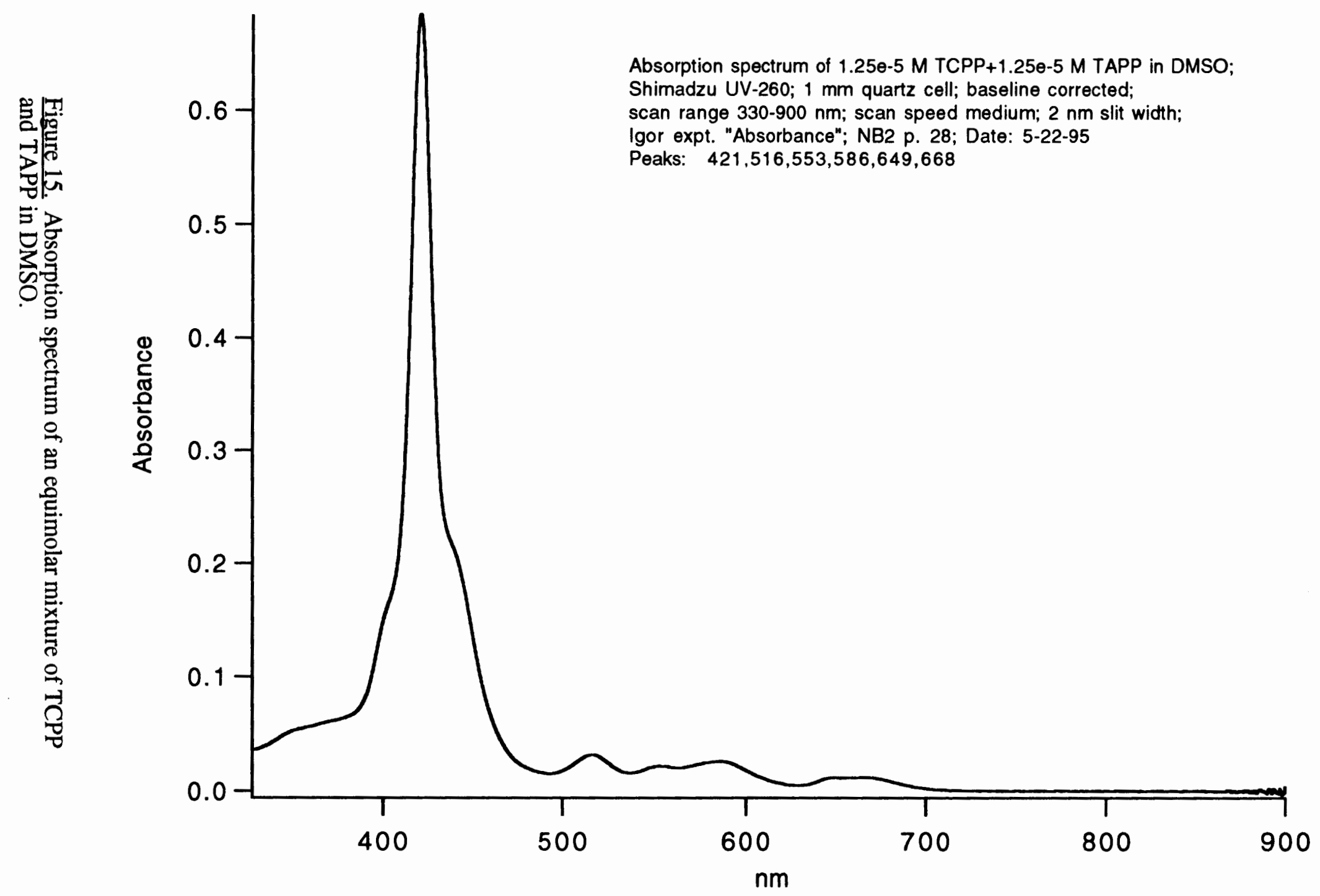


porphyrins is shown in Figure 15. It corresponds almost exactly to the sum of the spectra of the individual monomers. The Soret peak of TCPP is easily seen, and the broadening of the base is due to TAPP. The Q1 band of TCPP is little changed, but the other $\mathrm{Q}$ bands have all broadened. For analytical purposes, however, it was found that fluorescence spectroscopy was a much more sensitive and selective analytical technique for determining the presence of TCPP and TAPP in an unknown mixture, as described below.

Correlation between a solvent characteristic and the positions of the absorption maxima for TCPP and TAPP is difficult to find. The most polar protic solvents (water and methanol) move absorptions to shorter wavelengths, while polar aprotic solvents (DMSO and DMF) move peaks to their redmost positions. Other than this, no pattern is apparent.

The absorption spectrum of TMCPP is identical to that for TCPP in DMSO, and qualitatively similar in dichloromethane, in which TCPP is insoluble. TMCPP is insoluble in both water and methanol, so it is unfortunately impossible to determine what effects these solvents would have upon substitution of the carboxyl hydrogen by a methyl group.

\section{Fluorescence Spectroscopy}

Emission and excitation spectra for TCPP and TAPP in various solvents are also presented in the Appendix. Due to the fact that the absorbance of the reference dye in the fluorimeter cuts out at about $610 \mathrm{~nm}$, no excitation peaks could be seen beyond this wavelength, and so the last $\mathrm{Q}$ band could not be observed for either 
porphyrin. Otherwise, correlation between the excitation and absorption spectra is excellent for both porphyrins, as expected. Thus excitation at all absorbing wavelengths results in detectable emission at the same wavelength. However, the Soret peaks in the excitation spectra are less intense relative to the Q-bands, than in the absorption spectra. This could be due either to a lower fluorescence quantum yield for the Soret, or to an inner filter effect. In other words, since the absorptivity of the Soret band is so high $\left(\approx 10^{5} \mathrm{M}^{-1} \mathrm{~cm}^{-1}\right)$, absorption moves to the front of the sample cell, and less emission is detected at right angles to the cell. An inner filter effect was thought to be responsible for a similar discrepancy observed in the photocurrent action spectrum of TCPP metallated with zinc $^{43}$.

Remarkably, the emission spectrum of TCPP shows very little dependence on solvent. The first maximum (the $\mathrm{Q}_{(0,0)}$ transition) appears within the range 648-651 $\mathrm{nm}$ and the second $\left(\mathrm{Q}_{(0,1)}\right)$ within the range 713-717 $\mathrm{nm}$. The ratio of the two peak heights differs somewhat from solvent to solvent, from 3.1 in DMSO to 2.5 in ethyl acetate. In general, the more intense the $Q_{(0,0)}$ peak, the greater the $Q_{(0,0)}: Q_{(0,1)}$ ratio. This suggests that the variation is due to small differences in concentration rather than to solvent effects.

The emission of TAPP is much more variable, with the maximum appearing anywhere from 669 to $699 \mathrm{~nm}$. Usually only two transitions are discernible, but four can clearly be seen in ethyl acetate.

It has been found $\mathrm{d}^{76,77}$ that tetraphenylporphyrins with electron-donating para substituents on the phenyl rings undergo intramolecular charge transfer when the 
pyrrole nitrogens become protonated. This causes a large increase in total absorbance, and a broadening of both absorption bands. It was proposed that the phenyl rings adopt a quinonoid structure, shifting charge into the macrocycle, and bringing them into coplanarity with the macrocycle. This effect may result in substantial fluorescence quenching.

The integrated emission for TCPP in DMSO solution is 1.67 times greater than that for TAPP when each is excited at the optimum (Soret) wavelength. The ratio of absorbed fluxes at the optimum wavelengths (422 nm for TCPP and $441 \mathrm{~nm}$ for TAPP) is 1.53 , which means that the quantum efficiency for fluorescence is greater for TCPP than for TAPP with excitation at their respective Soret wavelengths. Equal integrated emission for equal concentrations of TCPP and TAPP was found to occur at an excitation wavelength of $432.5 \mathrm{~nm}$ in DMSO solution.

Emission spectra of TMCPP are also identical to those of TCPP in the same solvents. Methylation of all four carboxyl groups has very little effect on the excited state characteristics of the molecule.

Fluorescence spectroscopy was used to determine the presence of either porphyrin monomer in an unknown sample. Very dilute solutions could be tested, which made it ideal in analyzing fractions from a small chromatography column. Studies were carried out to determine the optimum conditions for clear detection of one monomer with minimal interference from the other. Optimum wavelengths vary somewhat with solvent, but in general, since TAPP absorbs much more strongly in the range $450-460 \mathrm{~nm}$, one sets the excitation wavelength in this range and looks for 
the emission signature of TAPP. TCPP contributes $8 \%$ of the total emission from a 1:1 solution of the two porphyrins in DMSO, with excitation at $450 \mathrm{~nm}$. TAPP contributes $15 \%$ of the total emission when excitation is at $416 \mathrm{~nm}$. In general, one may selectively excite TCPP by choosing a wavelength a few nanometers less than its Soret wavelength. In addition, significant emission from TCPP exists over the range $630-640 \mathrm{~nm}$, which is quite free of emission due to TAPP. An emission wavelength is chosen in this range, and the excitation spectrum is acquired, which will clearly show TCPP. Thus the presence of either of these porphyrins can be detected even if the other is in excess.

The dyad is expected to show some quenching of the emission from both moieties due to electron transfer. If electron transfer from TAPP to TCPP is $100 \%$ efficient, no emission will be observed at all. On the other hand, if energy transfer from TCPP to TAPP is extremely efficient, then the emission spectrum will be that of TAPP, while the excitation spectrum will look like the sum of those for both monomers. Not only would such an observation indicate the presence of both species, it would also strongly indicate that they are covalently linked.

As a blank experiment, the fluorescence properties of a solution of the free monomers in DMSO were observed. The emission spectrum with excitation at 450 $\mathrm{nm}$ appeared to be simply the sum of the emissions of the individual monomers at this wavelength (Figure 16). With excitation at $416 \mathrm{~nm}$, the spectrum is not quite what one would expect (Figure 17). The taller $Q_{(0,0)}$ peak for TCPP is red shifted slightly, and the $\mathrm{Q}_{(0,1)}$ band is broadened and slightly less intense. The total 


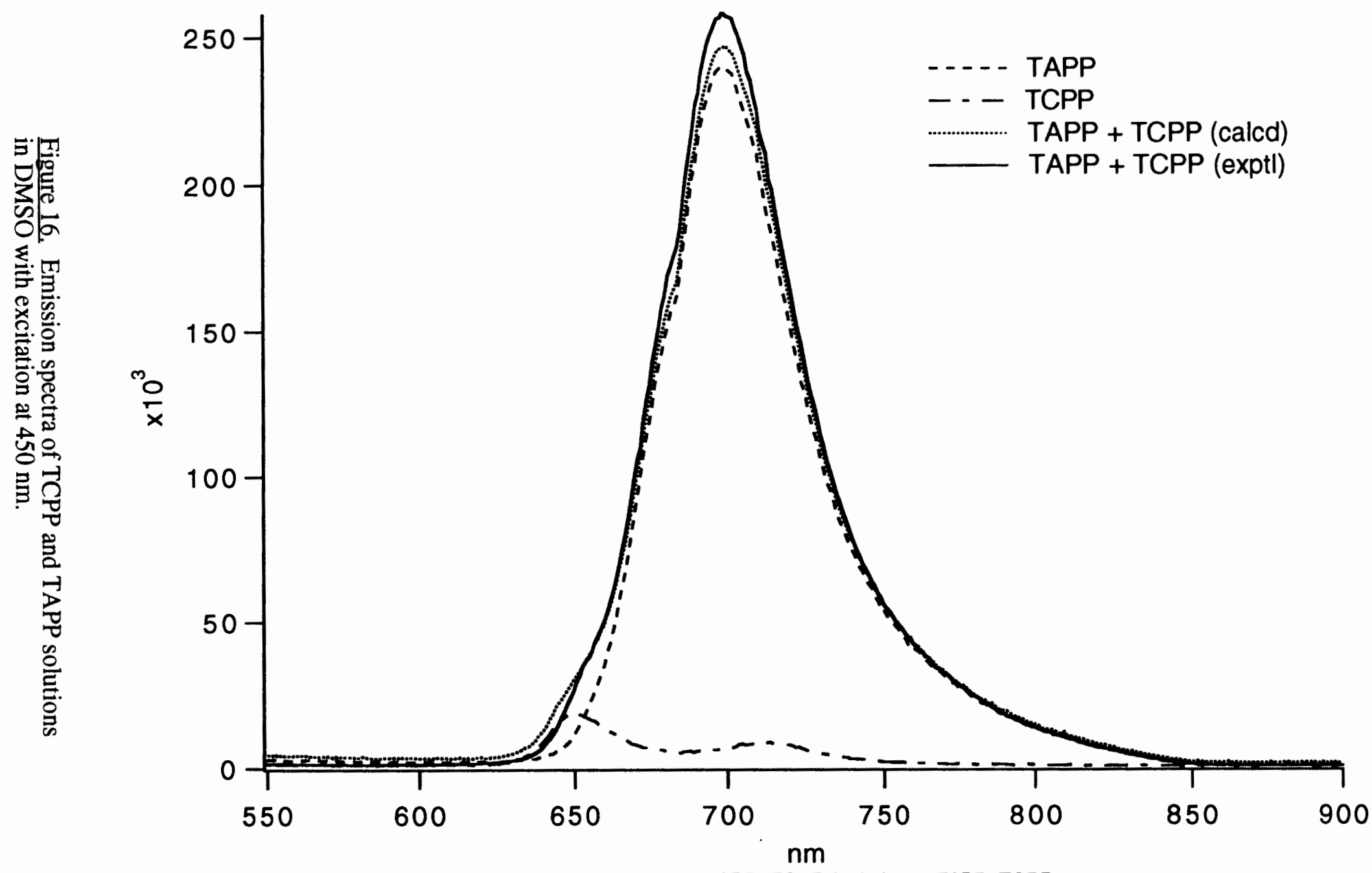

Emission spectra for TAPP (1e-6 M), TCPP (1e-6 M), TAPP+TCPP (calcd) and TAPP+TCPP

(exptl., 1e-6 M in each porphyrin) in DMSO, exc. at $450 \mathrm{~nm}$; SPEX Fluorolog; slits $0.5 \mathrm{~mm}$ (em.)

and $5.0 \mathrm{~mm}$ (exc.); $1 \mathrm{~nm}$ incr.; $0.5 \mathrm{sec}$. integ. time; PMT voltages 990/120; acq. mode 6; $1 \mathrm{~cm}$ quartz cell;

right-angle det.; corrected for solvent; Igor expt. "Emission"; NB2 p. 29; Date: 5-24-95 


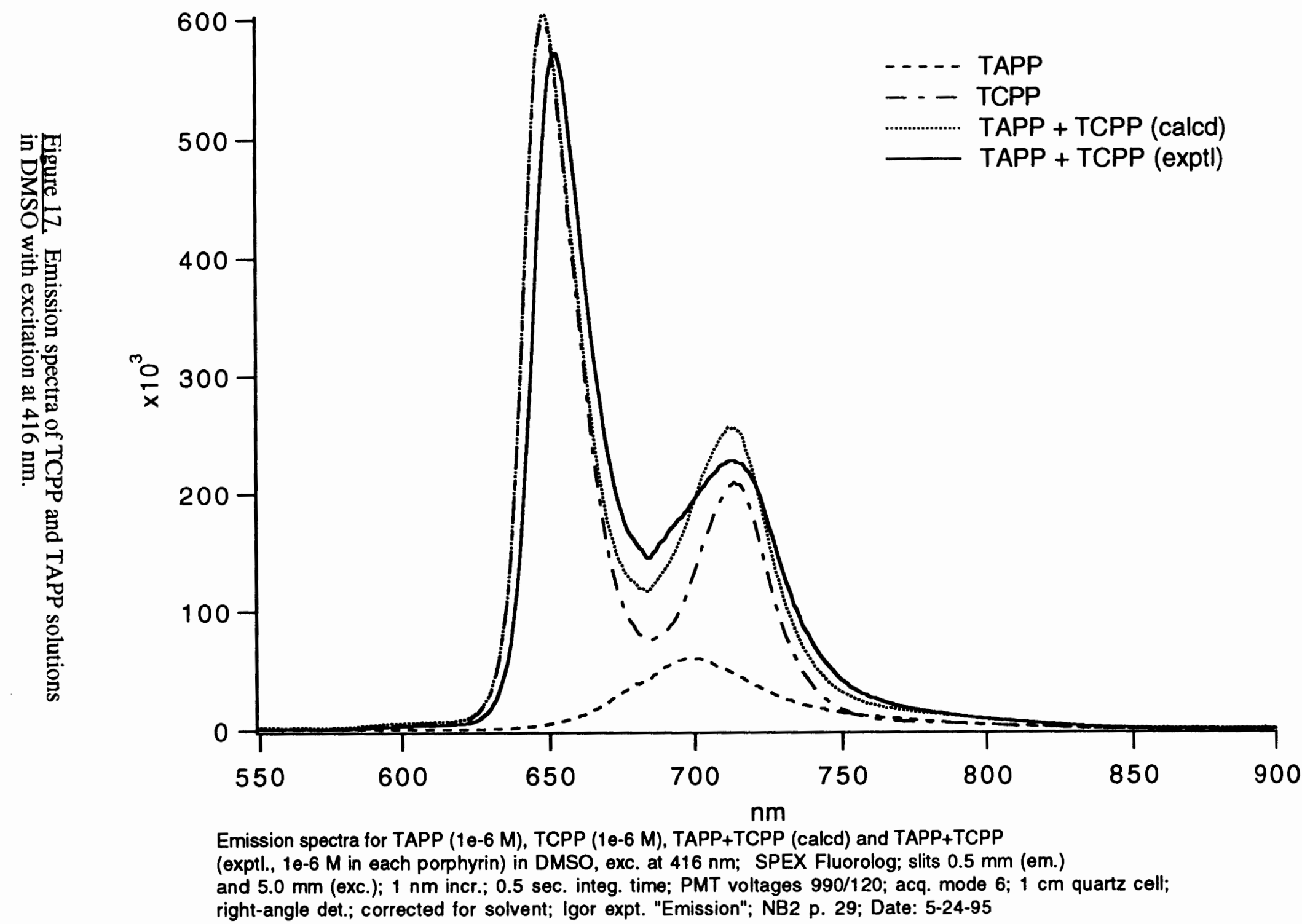


integrated emission differs by less than $0.2 \%$ from the sum of the two monomer spectra. No computer manipulation of monomer spectra could reproduce the observed spectrum. The peak shift is not consistent with simple emission quenching, but rather suggests complexation of TCPP. Self-aggregation is ruled out by the low concentration in a very strong solvent $(1 \mu \mathrm{M})$, and Beer's law plots for the absorbance of both porphyrins in DMSO was linear even at much higher concentrations $^{67}$. Complexation of TCPP and TAPP is possible, but would be expected to perturb the emission spectrum of both porphyrins, yet this was not observed in this experiment. Further study of this effect will be necessary before an accurate analysis of the dyad spectra can be made.

\section{NMR Spectroscopy}

NMR spectroscopy requires much more sample than electronic spectroscopy. A $10^{-4} \mathrm{M}$ solution is probably adequate, although many scans will be required to build up enough signal, but this concentration might be beyond the limit of solubility for the TCPP-TAPP dyad in many solvents. TCPP is quite soluble in water, but TAPP is insoluble. TAPP is soluble in chloroform, in which TCPP is insoluble. DMSO is an acceptable solvent for both monomers. DMF and pyridine are good solvents for the monomers, but deuterated DMF is expensive, and pyridine has resonances in the range $7-9 \mathrm{ppm}$, where porphyrin signals are found, which would be troublesome if it were of a low quality.

${ }^{1} \mathrm{H}$ NMR spectra of the monomers are included in the Appendix. These are consistent with similar spectra already acquired by this laboratory ${ }^{78}$. The dyad is 
expected to show shifts for both monomers in the resonances of the hydrogens on the phenyl rings nearest the amide bond, since this has been observed in other porphyrin dyads, and an amide proton signal should appear around $8-9 \mathrm{ppm}^{32,63}$.

An interesting effect was observed in the NMR spectrum of each monomer upon addition of $\mathrm{D}_{2} \mathrm{O}$ to the DMSO sample solutions: the resonance for the $\mathrm{B}$ hydrogens (on the pyrrole rings) collapsed into a low, broad singlet. Its integral remained unchanged relative to the other peaks in the spectrum, so it is not an exchange phenomenon. This effect was observed on another occasion, but could not be reproduced a third time. The suggested explanation is that the solutions in which resonance broadening was observed happened to be saturated with porphyrin, and addition of water caused some aggregation to occur. However, it is hard to see how porphyrins could aggregate so as only to affect the electronic environment of the $B$ hydrogens, and not the phenyl hydrogens ortho to the bond to the macrocycle. This phenomenon should also be explored further.

\section{CHROMATOGRAPHY}

An enormous amount of time was wasted, and experiments spoiled, by carrying out column chromatography using an improperly prepared stationary phase. This was by far the most time-consuming task of this entire project. A vital tip was learned from an expert at EM Separations ${ }^{79}$, which was not available in any of the chromatography textbooks consulted. The tip was that silica gel must be deactivated with water in order to reproduce results from thin layer chromatography. This holds 
true even for reverse-phase media, because only about $20-25 \%$ of the silanol groups are actually alkylated. In general, it was found that treatment of the adsorbent with water also reduced the band broadening of compounds containing free carboxyl or amine groups. Surprisingly, for separation of the methyl esters of TCPP, the silica gel required slurrying in acetone containing $15 \%$ water by weight of dry gel in order to get reproducible results and good recovery. The appropriate amount of water must be determined by trial and error for the separation of different compounds. It is important to realize that the gel must be deactivated with water before applying the sample. It was found in one experiment that porphyrins that had been applied to the untreated gel could not be removed even by Soxhlet extraction with $95 \%$ ethanol or pyridine.

Pretreatment of silica gel with water was found not to be necessary if acetic acid was used in the eluent. It was found that TCPP and TAPP could be separated on a silica gel column as follows: the silica gel was slurried in dichloromethane and the column poured. The sample was dissolved in dichloromethane containing $5 \%$ acetic acid by volume and applied to the column. The same solvent eluted TCPP as a tight green band. Methanol:dichloromethane (20:80) then brought down TAPP. Attempts at separating TCPP and TAPP on a silica gel HPLC column using the above solvents are being carried out by another graduate student in this laboratory. Success in this area would lend a tremendous advantage to analyzing reaction mixtures containing these porphyrins. 
For isolation of TMCPP and TrMCPP, the silica gel is deactivated with $15 \%$ water by weight of dry gel, in an acetone slurry. The acetone is allowed to evaporate overnight, or under an airstream. The gel is then slurried in dichloromethane and the column is poured. The sample is dissolved in 3:1 dichloromethane:ethyl acetate and applied to the column. This solvent will elute TMCPP and TrMCPP in well-resolved bands, although more ethyl acetate may be needed to bring down TrMCPP at an acceptable rate. Lower esters are left on top of the column; no conditions were determined for their recovery.

For separation of TCPP and TAPP on thin layer silica gel plates, a different approach was used. The plate was first pre-run in methanol, which seemed to deactivate it somewhat. Spots were applied, and the plate was developed using 2:1 ethyl acetate:dichloromethane. TAPP had the higher $\mathbf{R}_{\mathrm{f}}$.

For the esters of TCPP, 3:1 dichloromethane:ethyl acetate moved TMCPP and TrMCPP on an untreated plate. In order to resolve all of the esters, 1:1 acetone: dichloromethane was used as solvent.

It was noticed that after a spot of TAPP had thoroughly dried on a TLC plate, it could not be moved again. Whether this was due to binding of the porphyrin to the silica gel or to polymerization is unknown.

It is hard to predict whether the TLC spot for the dyad would show up between those for the monomers, or at a lower $\mathbf{R}_{\mathrm{f}}$. It is vital that the solvent be capable of moving it, since a spot left at the origin could equally well be polymeric material. If the dyad is synthesized with methylated carboxyl groups, it might be 
isolated on a silica gel column by using the 3:1 dichloromethane:ethyl acetate solvent containing some methanol, perhaps $20 \%$, which will probably be required to move the amine groups on the TAPP moiety.

\section{CONCLUSIONS}

The TCPP-TAPP dyad can be synthesized. The MALDI mass spectrum shows this, though it does not prove the nature of the linkage between the two monomers. What has been found in this research is that, since TAPP is such a poor nucleophile, strong activation of the carboxyl group is necessary. Reaction of TAPP with an acid chloride of TCPP in the presence of pyridine seems to work, but the use of thionyl chloride as an activating agent is troublesome, due to the problem of removing the $\mathrm{HCl}$ byproduct. Activation using triphenylphosphine and carbon tetrachloride may be a better method.

Protection of the carboxyl groups of TCPP is probably necessary to facilitate chromatography of the product mixture. Although TCPP can be moved on a silica gel column, this requires the use of acetic acid, which may be difficult to remove completely from the product. This lab has experienced problems with traces of propionic acid in commercial samples of TCPP, for whose synthesis it is the reaction solvent ${ }^{80}$. Protection of the free amine groups of TAPP would make chromatography easier, but would complicate the overall synthesis. TAPP can be moved on silica fairly easily using methanol in dichloromethane. 
MALDI-MS is probably the best way to prove that a product of the correct molecular weight has been created. FAB-MS (fast-atom bombardment mass spectroscopy) was carried out on the monomers and on some reaction mixtures, but only peaks due to the monomers were visible, and were far too weak to be useful. A careful NMR study may be expected to show a resonance between $8-9 \mathrm{ppm}^{32,63}$ to indicate an amide hydrogen. Cruder tests may be carried out using fluorescence spectroscopy to indicate whether a given solution contains both monomers, assuming that some emission could be observed even when the monomers are linked and undergoing electron transfer. If excitation of the donor led to the charge separated state extremely efficiently, but nonradiative charge recombination was very rapid, one would see no donor emission and would not be aware of its presence. Assuming that both TAPP and TCPP would emit detectably even when linked together, one can determine their presence using the procedures described above.

The possibility that TAPP by itself can function as the sensitizer by reacting it with a $\mathrm{TiO}_{2}$ electrode that has been treated with thionyl chloride should be explored. If the results are encouraging, then the next step would be to try to grow polymeric TAPP electrochemically from the monolayer already attached to the electrode. Ideally, one would try to link the polymer chain to both electrodes of the cell, thus assuring optimum contact of the polymer electrolyte with the electrodes. 


\section{CHAPTER III}

\section{EXPERIMENTAL}

\section{Materials and Methods}

Tetrakis-meso(4-carboxyphenyl)porphine was from Porphyrin Products, and tetrakis-meso(4-aminophenyl)porphine was from Midcentury. Both were used as received. Methanol was from Baker, HPLC grade; dichloromethane was from EM, HPLC grade; 1,2-dichloroethane was from Pierce, Sequanal grade; pyridine was from EM, spec-GC grade; DMSO, ethyl acetate, t-butyl methyl ether and toluene were from Aldrich, HPLC grade; DMF was from MCB, pesticide quality; other solvents and reagents are covered in the experimental details below.

Except where noted, thin layer chromatography was carried out on Whatman PE SIL G/UV silica gel plates. Column chromatography was performed using Merck

9385 silica gel (60 $\AA$ pore size, $230-400$ mesh) or cellulose (Merck 2331 or Sigma C-6413).

Reactions involving porphyrins were carried out under as little illumination as possible.

Instrumentation

UV-Visible electronic spectra were acquired on a Shimadzu UV-260 spectrophotometer using a $2 \mathrm{~nm}$ slit width, $1 \mathrm{~mm}$ or $1 \mathrm{~cm}$ quartz cells, and an 
appropriate reference solution.

Fluorescence excitation and emission spectra were acquired on a SPEX Fluorolog model 112 spectrophotometer using a 150W xenon lamp as light source, $0.5 \mathrm{~mm}$ slits for the spectrometer being scanned and $5.0 \mathrm{~mm}$ slits for the other. Right-angle detection was used in all experiments. Sample solutions were generally $1 \mu \mathrm{M}$. Excitation spectra were corrected for varying lamp intensity using a reference of rhodamine B in propylene glycol, whose absorption stops at about $610 \mathrm{~nm}$, and thus prevents observation of excitation peaks beyond this wavelength (such as the Q4 band for porphyrins). Most emission spectra were referenced against blank solvent. Photon-counting detection was employed, using a Hamamatsu R928 photomultiplier tube.

${ }^{1} \mathrm{H}-\mathrm{NMR}$ spectra were acquired on a Nicolet $500 \mathrm{MHz}$ spectrometer, a Bruker AMX-400 $400 \mathrm{MHz}$ spectrometer, or a Varian EM390 $90 \mathrm{MHz}$ instrument; chemical shifts are reported versus $\mathrm{Me}_{4} \mathrm{Si}$ as an internal standard.

FAB-MS spectra were acquired at the Oregon Graduate Institute by Lorne Isabelle using a VG 7070 spectrometer and a $6 \mathrm{kV}$ accelerating voltage. Solvent matrices were diethanolamine and triethanolamine.

MALDI-MS were acquired at Oregon State University by Lilo Barofsky using a noncommercial instrument. Matrices were 2,5-dihydroxybenzoic acid (DHB) and $2^{\prime}, 4^{\prime}, 6^{\prime}$-trihydroxyacetophenone monohydrate (THAP). 
Synthesis of terephthalanilic acid (I)

Into a $100 \mathrm{~mL}$ round-bottom flask equipped with a Teflon stirbar were placed $1.714 \mathrm{~g}(10.32 \mathrm{mmol})$ terephthalic acid (MCB), $0.981 \mathrm{~g}(10.53 \mathrm{mmol})$ aniline (Mallinckrodt, distilled at low pressure), $0.064 \mathrm{~g}(0.52 \mathrm{mmol}) 4$ dimethylaminopyridine (Aldrich), and $20 \mathrm{~mL}$ dichloromethane. The dichloromethane did not dissolve the reactants, so $20 \mathrm{~mL}$ of DMF were added. Dicyclohexylcarbodiimide (Aldrich, $2.128 \mathrm{~g}, 10.31 \mathrm{mmol}$ ) was dissolved in $20 \mathrm{~mL}$ DMF in an addition funnel, which was then attached to the round-bottom flask. The system was purged with nitrogen, and a drying tube filled with Drierite was fitted to the top of the addition funnel. The reaction flask was chilled in an ice bath to $0^{\circ}$, and the DCC solution was added dropwise, with stirring, over a period of 5 minutes. The mixture was stirred an additional 5 minutes, after which the ice bath was removed. The reaction flask was protected from light, and left stirring for 18.5 hours.

The solvents were removed by vacuum, leaving an off-white solid. This solid was extracted with $1 \% \mathrm{NaOH}$ and filtered, leaving a beige-colored solid residue $(2.799 \mathrm{~g})$. The filtrate was acidified with $1 \mathrm{M} \mathrm{HCl}$ to $\mathrm{pH} 2$, and filtered to obtain a white solid $(1.362 \mathrm{~g})$. The two solid residues were dried at $100^{\circ}$ for 2 hours.

TLC on Kieselgel 60778 plates using pyridine as eluent showed that the product contained some unreacted terephthalic acid. The base-insoluble residue did not move. This residue decomposed in a melting point capillary at $225^{\circ}$. The rest remained whitish and melted around $270^{\circ}$. This residue probably contained the 
dicyclohexylurea byproduct (lit. mp 232-233 ${ }^{\circ}$ ) and the trimer. The white product decomposed at $310^{\circ}$ (terephthalanilic acid lit. mp 313-314 ${ }^{\circ 2}$; terephthalic acid lit. subl. $\left.402^{\circ 83}\right)$.

The IR spectrum (KBr pellet) of the base-insoluble residue was quite similar to that published for DCU (Sadtler). The IR spectrum of the product showed mainly peaks characteristic of terephthalic acid.

${ }^{1} \mathrm{H}-\mathrm{NMR}$ of the product (90 MHz, pyridine- $\mathrm{d}_{6}$, external TMS) showed the aniline ring proton signals between $7.2-7.6 \mathrm{ppm}$, the terephthalanilic acid ring protons between 8.2-8.5 ppm, the amide proton at $11.2 \mathrm{ppm}$, and the carboxylic acid proton at $14.8 \mathrm{ppm}$. A singlet due to unreacted terephthalic acid appeared at $8.6 \mathrm{ppm}$. No signals due to the undesired $\mathrm{N}$-acylurea were evident. Integration of the amide proton peak and the terephthalic acid singlet indicated an approximate composition of $40 \%$ terephthalanilic acid and $60 \%$ terephthalic acid. This indicated an overall product yield of about $25 \%$ (from terephthalic acid).

The base-insoluble residue proved to be insoluble in all other available NMR solvents as well, so no NMR analysis was possible.

Synthesis of terephthalanilic acid (II)

Into the same reaction assembly described above were placed $1.723 \mathrm{~g}(10.37$ mmol) terephthalic acid, $0.966 \mathrm{~g}(10.37 \mathrm{mmol})$ aniline, and $15 \mathrm{~mL}$ pyridine. DCC $(2.198 \mathrm{~g}, 10.65 \mathrm{mmol})$ was dissolved in $15 \mathrm{~mL}$ pyridine in the addition funnel, which was topped with a calcium chloride drying tube, and fitted to the round-bottom flask. The DCC solution was added under the same conditions as above, over a period of 
20 minutes. After an hour of stirring, the reaction mixture had turned milky white with precipitated material. After a total of 4.25 hours of stirring, the mixture was worked up as described above.

The $500 \mathrm{MHz}$ NMR spectrum of the product $\left(\mathrm{DMSO}-\mathrm{d}_{6}\right.$ ) showed the same signals as before, in addition to some peaks between 1-2 ppm which were probably due to the $\mathrm{N}$-acylurea. Integration of the peaks yielded an approximate composition for this mixture of $35 \%$ terephthalanilic acid, $60 \%$ terephthalic acid, and $5 \% \mathrm{~N}$ acylurea. This indicated an overall yield of $25 \%$ terephthalanilic acid from terephthalic acid.

\section{Synthesis of TCPP-TAPP dyad (DCC method)}

Into a $500 \mathrm{~mL}$ round-bottom flask containing a Teflon stirbar were placed 50 $\mathrm{mg}(0.063 \mathrm{mmol})$ of TCPP, $43 \mathrm{mg}(0.063 \mathrm{mmol}) \mathrm{TAPP}$, and $300 \mathrm{~mL}$ of DMF. The mixture was sonicated for 15 minutes. Dicyclohexylcarbodiimide (7 mg, 0.032 mmol) in $10 \mathrm{~mL}$ DMF was added dropwise at room temperature from an addition funnel topped with a $\mathrm{CaCl}_{2}$ drying tube. The mixture was stirred for 3 days, after which the solvent was removed by vacuum, to leave a nearly black solid (dry wt. $0.2665 \mathrm{~g}$ ). The solid was extracted with $\mathrm{CHCl}_{3}$ (Soxhlet) for 29 hours to preferentially remove unreacted TAPP. The material in the thimble (purple, dry wt. 0.1038 g) was chromatographed over silica gel (Analab 30-60 mesh) using 110:30:1 toluene:methanol:formic acid as solvent ${ }^{84}$, and increasing the concentrations of methanol and formic acid to bring down more material. Six fractions were obtained (total dry wt. $0.199 \mathrm{~g}$ ). After rotary evaporation of solvent, it was found that some 
of the residues were green, suggesting protonation of the porphyrin pyrrole nitrogens by the formic acid. These fractions were washed with $\mathrm{pH} 4$ buffer, followed by distilled water to remove any buffer salts.

UV-Vis spectra $\left(\lambda_{\max }, D M F\right):$ fraction $1: 417,513,548,588,646 \mathrm{~nm}$; fraction $2: 418,514,548,588,646 \mathrm{~nm}$; fraction $3: 418,516,549,588,647 \mathrm{~nm}$; fraction 4: 428, $499 \mathrm{~nm}$; fraction 5: 428, $560,600 \mathrm{~nm}$; fraction 6: 428, $499 \mathrm{~nm}$. Fractions 4-6 had very low solubility, and their absorbance spectra were weak.

The monomers and fractions 3-6 were subjected to FAB-MS analysis in a variety of solvents, of which triethanolamine and diethanolamine proved to be the best. TCPP was detected (weakly) using negative ion detection, while TAPP showed up best with positive ion detection. The spectra for fractions 3-6 showed ion peaks at $\mathrm{m} / \mathrm{z}=421$ with negative ion detection, and at $\mathrm{m} / \mathrm{z}=399$ with positive ion detection. This was consistent in a variety of solvent matrices. These peaks did not show up in the spectra of the monomers, and fractions or multiples of these masses did not appear in any spectra. The peak at $\mathrm{m} / \mathrm{z}=399$ was sometimes the strongest signal in the spectrum, even stronger than the solvent. This suggests the presence of a lower molecular weight impurity or reaction byproduct rather than a multiplyionized porphyrin.

Fractions 1-3 were judged to be largely TCPP on the basis of the absorbance spectra and chromatography characteristics, while fractions 4-6 were judged to be polymeric material, perhaps poly-TAPP, on the basis of low solubility. 
TCPP-TAPP linkage using a Merrifield resin

TCPP $(14.3 \mathrm{mg} .18 .1 \mu \mathrm{mol})$ was dissolved in $18 \mathrm{~mL}$ DMF (MCB, fractionated under vacuum, treated overnight with activity I alumina, fractionated again, and stored over molecular sieves) in a silanized ${ }^{71} 50 \mathrm{~mL}$ three-necked flask. 2-Chlorotrityl chloride resin (55 mg, $36 \mu \mathrm{mol}$ active chloride sites; Advanced ChemTech) was suspended in $2 \mathrm{~mL} \mathrm{DMF}$ and added to the TCPP solution. $\mathrm{A} \mathrm{CaCl}_{2}$ drying tube was attached on one neck, a rubber septum on another, and the third was tightly stoppered. Diisopropylethylamine $(3.0 \mu \mathrm{L}, 18 \mu \mathrm{mol}$, Aldrich) was injected through the septum. The solution was left for 3 hours, with occasional swirling, after which it was filtered using a coarse, silanized frit. The resin was washed three times with DMF and three times with methanol (Baker HPLC), after which the resin turned green, due to protonation of the porphyrin pyrrole nitrogens by $\mathrm{HCl}$ liberated by reaction of the methanol with unreacted trityl chloride groups. The resin was washed three more times with DMF (resin turned back to purplish), and three times with 1,2dichloroethane (DCE).

TAPP $(45.8 \mathrm{mg}, 67.9 \mu \mathrm{mol})$ and diisopropylcarbodiimide $(20 \mu \mathrm{L}, 128 \mu \mathrm{mol}$, Aldrich) were dissolved in $70 \mathrm{~mL} \mathrm{DCE}$ in a silanized $250 \mathrm{~mL}$ three-necked flask. The TCPP-resin was added to this solution using DCE. The mixture was allowed to sit in the dark, with occasional swirling, for 6 hours, after which it was filtered as above, washing with DCE and DMF until the filtrate was colorless.

The cleavage solution was prepared from acetic acid, trifluoroethanol (both from Aldrich) and DCE (1:1:8). Ten milliliters of this solution were added to the 
resin in the filter funnel, which was tilted to prevent the solution from draining out. After 2 hours, very little material had come off the resin. Washing with DMF resulted in a colorless filtrate. A 1:1:3 solvent mixture was used, and finally neat trifluoroacetic acid (Aldrich), but neither removed a significant amount of material.

The bright green TFA filtrate could not be evaporated to dryness. Addition of aqueous $\mathrm{NaOH}$ resulted in two phases, but the aqueous phase did not decolorize, and the organic phase remained green. The organic phase was pipetted off and aqueous ammonia added, giving a brownish-yellow solution which could not be evaporated to dryness either. A few drops of $0.1 \mathrm{M} \mathrm{HCl}$ were added, followed by 10 $\mathrm{mL}$ of distilled water. An absorption spectrum of the yellowish solution ( $\mathrm{pH} \mathrm{6}$ ) showed a low, very broad band centered at about $410 \mathrm{~nm}$ and a small peak at 521 nm. An emission spectrum showed weak peaks suggestive of TCPP, plus a very large peak at about $760 \mathrm{~nm}$, perhaps due to phosphorescence. The solution was subjected to strong vacuum (liquid $\mathrm{N}_{2}$ trap) and pumped down to 10 microns pressure. A colorless crystalline material precipitated, and the solution became yellowish in color. Dichloromethane was added, but little dissolved. An absorption spectrum of the yellowish DCM solution showed peaks at 417 and $462 \mathrm{~nm}$, and a low broad absorption at about $720 \mathrm{~nm}$, inconsistent with either monomer porphyrin. Addition of ammonia to this solution produced no color change, so the spectrum was not that of a protonated porphyrin. The crystalline material was very soluble in water. 
The resin was dried by vacuum, and returned to a red-brown color. However, addition of trifluoracetic acid did not turn the resin green. After two days, the TFA solution was faintly brownish, and the resin had turned black. The resin was filtered and washed with DMF and DCE, but the filtrates were colorless. The resin was then soaked for two days in a solution of $30 \mathrm{~mL} \mathrm{DCE}, 1 \mathrm{~mL}$ acetic acid and $1 \mathrm{~mL}$ of trifluoroethanol. The resin was filtered, and the faintly brownish filtrate was evaporated to dryness. The residue was washed with hexane and then with water. UV-Vis $\left(\lambda_{\max }\right.$, DMSO): $422,513,550,588,646 \mathrm{~nm}$; emission (exc. $\left.390 \mathrm{~nm}\right): 651$, $716 \mathrm{~nm}$.

The resin was ground up in a mortar and added to DMSO. Very little material dissolved, and the weak absorption spectrum showed only one peak at 420 nm, suggesting more TCPP.

\section{Test of cleavage of TCPP from Merrifield resin}

TCPP ( $5 \mathrm{mg}, 6 \mu \mathrm{mol}$ ) was dissolved in $6 \mathrm{~mL}$ DMF in a silanized $50 \mathrm{~mL}$ three-necked flask. Diisopropylethylamine $(3.0 \mu \mathrm{L}, 18 \mu \mathrm{mol})$ and $3 \mathrm{~mL} \mathrm{DCE}$ were added. 2-Chlorotrityl chloride resin (38 mg, $25 \mu \mathrm{mol}$ active sites) was suspended in $2 \mathrm{~mL}$ DCE and added to the TCPP solution. The vessel was flushed with nitrogen, topped with a $\mathrm{CaCl}_{2}$ drying tube, protected from light, and allowed to sit overnight with agitation provided by the vibrations of a magnetic stirrer set on full speed (no stirbar). No noticeable decolorization was noticed, nor after 2 hours of sonication. About $1 \mathrm{mg}$ of 4-dimethylaminopyridine was added, but after 6 days, there was still little decolorization of the solution. The resin was filtered and washed with DCE 
and DMF. Washing with methanol did not turn the resin green, indicating that there were no more active sites left. The resin was then treated with a 1:1:3 solution of acetic acid:trifluoroethanol:DCE, which turned the resin an olive color. After about an hour, the resin was bright green, indicating that the porphyrin was in a very hindered environment. Addition of DMSO turned the solution pink, but the resin remained green until the next day. The resin was filtered and washed with a solution of $4: 1: 3: 3$ acetic acid:water:DCE:DMSO. The filtrate was evaporated to a volume of $6.25 \mathrm{~mL}$, after which no more solvent could be removed. An absorption spectrum indicated a TCPP concentration of $20 \mu \mathrm{M}$, or $1.3 \times 10^{-7} \mathrm{~mol}$. The resin was still pink, indicating incomplete recovery. Thus $2 \%$ of the TCPP used was recovered, and could be recovered from only $0.5 \%$ of the active sites on the resin.

\section{$\underline{\text { Adsorption of TCPP onto } \mathrm{TiO}_{2}}$}

$\mathrm{TiO}_{2}$ powder $(5.3 \mathrm{mg}$, Degussa $\mathrm{P}-25)$ was placed in a $50 \mathrm{~mL}$ round-bottom flask containing a stirbar. Methanol $(1 \mathrm{~mL})$ was added and the suspension was stirred briefly. Then $5.00 \mathrm{~mL}$ of a $9.383 \times 10^{-5} \mathrm{M}$ stock solution of TCPP in methanol were added (469 nmol TCPP), and the suspension was stirred overnight. The mixture was centrifuged, the $\mathrm{TiO}_{2}$ was washed with methanol, and the washings were combined with the supernatant. Absorption spectra of the stock and supernatant solutions indicated that $147 \mathrm{nmol}$ of TCPP had been adsorbed, or $31 \%$ of the available material. Using a BET surface area of $50 \mathrm{~m}^{2} / \mathrm{g}$ for the $\mathrm{TiO}_{2}{ }^{85}$, this indicates a surface coverage of $55 \mathrm{pmol} / \mathrm{cm}^{2}=44 \mathrm{ng} / \mathrm{cm}^{2} \approx 300 \AA^{2} /$ molecule. One TCPP 
molecule lying flat covers approximately $310 \AA^{2}$. This coverage is also equivalent to $22 \mathrm{mg} \mathrm{TCPP} / \mathrm{g} \mathrm{TiO}_{2}$.

The $\mathrm{TiO}_{2}$ (grayish when dry, pinkish when wet) was treated with $2 \mathrm{M} \mathrm{NaOH}$ to remove the porphyrin. The supernatant was neutralized with $\mathrm{HCl}$ and an absorption spectrum taken, along with a spectrum of $50 \mu \mathrm{M}$ TCPP in $2 \mathrm{M} \mathrm{NaCl}$ for reference (the porphyrin is significantly dimerized at this high ionic strength). The calculated recovery was $108 \mathrm{nmol}$, or $74 \%$.

\section{Addition of TAPP to $\mathrm{TiO}_{2}$ treated with $\mathrm{SOCl}_{2}$}

$\mathrm{TiO}_{2}(13.1 \mathrm{mg})$ and 15 drops of $\mathrm{SOCl}_{2}$ were stirred overnight in a stoppered centrifuge tube. The $\mathrm{TiO}_{2}$ was dried at $50^{\circ}$ for 10 minutes, and then a solution of $0.5 \mathrm{mg}$ TAPP in $1.5 \mathrm{~mL}$ THF was added, followed by 10 minutes of sonication. The mixture was then stirred for 4 hours. The red supernatant solution was removed, leaving the bluish gray $\mathrm{TiO}_{2}$. Methanol did not immediately desorb any material, but after two days, the methanolic solution was faintly violet-colored. An absorption spectrum showed a peak at $417 \mathrm{~nm}$ and a very broad one at about $579 \mathrm{~nm}$, superimposed on a scattering background. The methanol was pumped off and $2 \mathrm{~mL}$ of $2 \mathrm{M} \mathrm{HCl}$ were added. The $\mathrm{TiO}_{2}$ did not turn green quickly, but the supernatant became faintly greenish upon standing overnight. Its absorption spectrum showed peaks at 440, 593 and $648 \mathrm{~nm}$. The $\mathrm{HCl}$ solution was pumped off and $1 \mathrm{~mL}$ of pyridine was added. This failed to remove anything from the $\mathrm{TiO}_{2}$. 


\section{TCPP-TAPP linkage on $\mathrm{TiO}_{2}$ using $\mathrm{SOCl}_{2}$}

$\mathrm{TiO}_{2}(5.6 \mathrm{mg})$ was stirred for 25 hours in a centrifuge tube containing 5.00 $\mathrm{mL}$ of the stock TCPP solution (469 nmol TCPP), as described in the above experiment. The calculated surface coverage was $56 \mathrm{pmol} / \mathrm{cm}^{2}$. The $\mathrm{TiO}_{2}$ with 158 nmol TCPP adsorbed was dried at $115^{\circ}$ for 15 minutes, and then $2 \mathrm{~mL}$ pyridine, 50 $\mu \mathrm{L}$ DMF and $125 \mu \mathrm{L}$ thionyl chloride (Aldrich, $1.7 \mu \mathrm{mol}$ ) were added, and the mixture was stirred overnight in the centrifuge tube. Some material desorbed from the $\mathrm{TiO}_{2}$ (brownish green), so the mixture was centrifuged and the $\mathrm{TiO}_{2}$ washed twice with pyridine. Then TAPP ( $1 \mathrm{mg}, 1.5 \mu \mathrm{mol})$ in $1 \mathrm{~mL}$ pyridine was added to the $\mathrm{TiO}_{2}$, and the mixture was stirred for three days, followed by centrifugation and washing with 1,2-dichloroethane. The $\mathrm{TiO}_{2}$ (greenish brown) was treated with $2 \mathrm{~mL}$ of $2 \mathrm{M} \mathrm{NaOH}$, and sonicated 5 minutes, but very little material desorbed. The solution was made $4 \mathrm{M}$ in hydroxide by the addition of $\mathrm{NaOH}$ pellets, followed by 10 minutes of sonication. Very little material desorbed. The supernatant was neutralized with $\mathrm{HCl}$, and an absorption spectrum taken. Only a weak Soret band at $411 \mathrm{~nm}$ was visible.

\section{TCPP-TAPP linkage on $\mathrm{TiO}_{2}$ using diisopropylcarbodiimide}

$\mathrm{TiO}_{2}(20.7 \mathrm{mg})$ and $5.00 \mathrm{~mL}$ of TCPP stock solution (469 nmol TCPP) were stirred in a centrifuge tube for 3 days. The supernatant was substantially decolorized. After centrifugation and washing with DMF, TAPP (3.2 mg, $4.7 \mu \mathrm{mol})$ in $2 \mathrm{~mL}$ DMF was added, along with a stirbar and $10 \mu \mathrm{L}$ diisopropylcarbodiimide (64 $\mu \mathrm{mol}$, Aldrich). The mixture was stirred overnight. The supernatant became 
green, but the $\mathrm{TiO}_{2}$ was pink. The mixture was centrifuged and the $\mathrm{TiO}_{2}$ washed with DMF until the supernatant was colorless, and then with methanol. The $\mathrm{TiO}_{2}$ was then dried at $50^{\circ}$ in a vacuum oven for 30 minutes. Three $\mathrm{mL}$ of $2 \mathrm{M} \mathrm{NaOH}$ were added, and the mixture was sonicated 20 minutes, then centrifuged. The $\mathrm{TiO}_{2}$ was washed once more with $\mathrm{NaOH}$ solution, and the supernatants combined and neutralized with $\mathrm{HCl}$. The absorption spectrum of the resulting solution had peaks at $408,527,564,594$ and $651 \mathrm{~nm}$. The emission spectrum (exc. at $521 \mathrm{~nm}$ ) had a peak at $664 \mathrm{~nm}$ with a shoulder at $688 \mathrm{~nm}$. These were consistent with the presence of TCPP monomer.

\section{TCPP-TAPP linkage on $\mathrm{TiO}_{2}$ using $\phi_{3} \mathrm{P}$ and $\mathrm{CCl}_{4}$}

$\mathrm{TiO}_{2}(9.7 \mathrm{mg})$ and $2.8 \mathrm{~mL}$ TCPP stock solution (263 nmol TCPP) were stirred in a centrifuge tube overnight. The pink $\mathrm{TiO}_{2}$ was centrifuged and washed with methanol until the supernatant was colorless. The $\mathrm{TiO}_{2}$ was transferred to a 10 $\mathrm{mL}$ round-bottom flask with $2 \mathrm{~mL} \mathrm{1,2-dichloroethane.} \mathrm{Triphenylphosphine}$ (Eastman, $1 \mathrm{mg}, 4 \mu \mathrm{mol}), \mathrm{CCl}_{4}(\mathrm{MCB}, 200 \mu \mathrm{L}, 2.1 \mu \mathrm{mol})$ and TAPP (1.8 $\mathrm{mg}, 2.7$ $\mu \mathrm{mol})$ dissolved in $1.5 \mathrm{~mL} \mathrm{DCE}$ were added, and the mixture was refluxed for four hours, and then allowed to sit for three days at room temperature. The $\mathrm{TiO}_{2}$ (brown) was washed with methanol until the supernatant was colorless, and then treated with $2 \mathrm{M} \mathrm{NaOH}$, but the amount of material desorbed was negligible. DMSO was added, and some material desorbed, turning the supernatant green, indicating deprotonation of the pyrrole nitrogens. After centrifugation, the supernatant was neutralized with $\mathrm{HCl}$, becoming red-brown in color. The absorption spectrum of this solution had a 
very broad peak at $421 \mathrm{~nm}$ with a shoulder at $455 \mathrm{~nm}$, and another peak at $746 \mathrm{~nm}$. The emission spectrum was flat (excitation at $450 \mathrm{~nm}$ ), and the excitation spectrum was also flat with detection at $745 \mathrm{~nm}$.

\section{TCPP-TAPP linkage on $\mathrm{TiO}_{2}$ via pyrolysis}

$\mathrm{TiO}_{2}(20.0 \mathrm{mg})$ and $2.00 \mathrm{~mL}$ of a $2.0 \times 10^{-4}$ solution of TCPP in methanol (400 nmol TCPP) were stirred in a centrifuge tube for three days. After centrifugation and washing, TAPP $(1.1 \mathrm{mg}, 1.6 \mu \mathrm{mol})$ in $2 \mathrm{~mL}$ methanol was added to the $\mathrm{TiO}_{2}$. The mixture was transferred to a watch glass by pipet, and the methanol allowed to evaporate. The watch glass was then placed in a sintering furnace at $200^{\circ}$ for 20 hours. The mixture was transferred to a centrifuge tube with DMF, and washed with DMF to remove excess TAPP, and then with methanol. The $\mathrm{TiO}_{2}$ was gray. $\mathrm{NaOH}(2 \mathrm{M})$ did not desorb any material, but addition of DMSO to the base solution resulted in some desorption, turning the supernatant and the $\mathrm{TiO}_{2}$ bright green. The supernatant was removed via centrifugation and neutralized with $\mathrm{HCl}$. The absorption spectrum showed some scattering, but a Soret peak was visible at 419 $\mathrm{nm}$, and Q-bands at 515, 557, 596 and $643 \mathrm{~nm}$. The excitation spectrum (det. at 650 $\mathrm{nm})$ had peaks at $414,517,554$ and $582 \mathrm{~nm}$. These spectra were consistent with TCPP monomer.

TCPP-TAPP coupling with diisopropylcarbodiimide (DIC)

To a small vial was added $1.3 \mathrm{mg}$ TCPP (1.6 $\mu \mathrm{mol}), 1.5 \mathrm{mg}$ TAPP $(2.2$ $\mu \mathrm{mol}), 3 \mathrm{~mL}$ DMSO and a stirbar. After the porphyrins dissolved, $125 \mu \mathrm{L}$ DIC 
( $800 \mu \mathrm{mol}$ ) was added. The vial was capped and the contents stirred for 3 days. The contents were transferred to a centrifuge tube, the porphyrins were precipitated with $2 \mathrm{M} \mathrm{NaCl}$ and collected by centrifugation. The purple residue was washed twice with water. UV-Vis ( $\lambda_{\max }$, DMSO): $420,440(\mathrm{sh}), 515,551,586,647,666$ $\mathrm{nm}$, consistent with both TAPP and TCPP. Excitation (DMSO, det. $640 \mathrm{~nm}$ ): 420, 516, 548, $589 \mathrm{~nm}$, consistent with TCPP; (det. $760 \mathrm{~nm}$ ): 520, 576, 601 (sh) nm, consistent with TAPP (Soret off-scale). Emission spectrum (exc. $410 \mathrm{~nm}$ ): 649 and $712 \mathrm{~nm}$, consistent with TCPP; (exc. $450 \mathrm{~nm}$ ): $682(\mathrm{sh})$ and $699 \mathrm{~nm}$, consistent with TAPP. NMR (400 MHz, DMSO-d $\left.)_{6}\right):-2.95$ (s, TCPP pyrrole NH), -2.74 (s,br, TAPP pyrrole NH), 1.01 (d, iPr methyl), 1.31 (d, iPr methyl), 5.59 (s,br, TAPP $\mathrm{NH}_{2}$ ), 7.01 (d, TAPP phenyl $\mathrm{CH}$ ), 7.85 (d, TAPP phenyl $\mathrm{CH}$ ), 7.93 (d, activated TCPP phenyl $\mathrm{CH}$ ), 8.26 (d, activated TCPP phenyl $\mathrm{CH}$ ), 8.31 (s, unreacted TCPP phenyl $\mathrm{CH}$ ), 8.81 (s, TCPP pyrrole $\mathrm{CH}$ ), 8.88 (s, TAPP pyrrole $\mathrm{CH}$ ). Addition of $\mathrm{D}_{2} \mathrm{O}$ did not remove the singlet at $8.31 \mathrm{ppm}$, so this was not an amide proton signal, but was due to unreacted TCPP. Both of the pyrrole $\mathrm{CH}$ signals broadened into a singlet.

\section{Synthesis of TCPP-tetraacid chloride (TCCPP)}

In a typical procedure, $50 \mathrm{mg}$ of TCPP were weighed into a small roundbottom flask containing a stirbar. Thionyl chloride (Aldrich, $10 \mathrm{~mL}$ ) was added. A reflux condenser was attached, and the mixture brought to gentle reflux with stirring. The solution became green after a few minutes. After a few hours, the excess thionyl chloride was removed by vacuum. The green residue was then placed in a vacuum oven at $60^{\circ}$ and approximately 0.1 torr overnight. The final material was 
metallic blue. No characterization was undertaken; the material was used as is in further reactions.

\section{TCPP-TAPP polymer}

A small vial containing a stirbar was dried at $110^{\circ}$. TCCPP (1.6 mg, 1.9 $\mu \mathrm{mol})$ and TAPP $(1.2 \mathrm{mg}, 1.9 \mu \mathrm{mol})$ were added, and the vial was set in an ice bath. While stirring, $2 \mathrm{~mL}$ of dry pyridine were added dropwise. The mixture was allowed to stir for 4 days. Much precipitation occurred. Methanol was added to destroy any unreacted acid chloride groups, and then the vial was heated gently under an airstream to remove solvents. The brownish black residue was then dried under vacuum at $100^{\circ}$ for 2 hours. Very little material could be dissolved in DMSO- $\mathrm{d}_{6}$, and the resulting spectrum showed weak peaks due to pyridine, and one small multiplet at $7.72 \mathrm{ppm}$.

\section{Synthesis of TCPP-methyl esters}

To approximately $3 \mathrm{mg}$ TCCPP mixture (see above) in a $25 \mathrm{~mL}$ round-bottom flask containing a stirbar was added $10 \mathrm{~mL}$ methanol and 2 drops pyridine. The flask was stoppered and stirred overnight. The solvent was removed under vacuum, and the brownish purple residue was dried under vacuum at $40^{\circ}$ for 1 hour. NMR (500 MHz, DMSO-d $\left.\mathrm{d}_{6}\right):-3.0$ (weak,br pyrrole $\left.\mathrm{NH}\right), 4.05\left(\mathrm{~s}, \mathrm{CH}_{3} \mathrm{O}\right), 7.89$ (t, phenyl $\mathrm{CH}), 8.84$ (s, pyrrole $\mathrm{CH}$ ). TLC (silica gel, 1:1 DCM:acetone) showed 5 spots, indicating the presence of TCPP and each of the four possible methylated products. 
The tetramethyl ester (TMCPP) and trimethyl ester (TrMCPP) were isolated via silica gel chromatography using 3:1 DCM:ethyl acetate eluent. The silica gel was first deactivated by slurrying it in acetone to which $15 \%$ water by weight of dry gel had been added. The acetone was evaporated under an airstream, and the gel was then slurried in dichloromethane for pouring the column.

\section{Synthesis of TCPP-TAPP dyad from TCCPP}

A TCCPP mixture $(61.1 \mathrm{mg}$, see above) was placed in a $100 \mathrm{~mL}$ roundbottom flask containing a stirbar. 1,2-Dichloroethane (DCE, $40 \mathrm{~mL}$ ) was added, and the flask was sealed with a rubber septum and sonicated 20 minutes. While stirring under a stream of dry nitrogen, $5.7 \mu \mathrm{L}$ methanol $(140 \mu \mathrm{mol})$ and $57 \mu \mathrm{L}$ pyridine (700 $\mu \mathrm{mol}$ ) were added via syringe through the septum. After 50 minutes, $95.4 \mathrm{mg}$ TAPP (141 $\mu \mathrm{mol})$ dissolved in $50 \mathrm{~mL}$ DCE were added. The mixture was stirred under a stream of nitrogen for one day, and then the nitrogen flow was stopped. Stirring continued another 4 days, after which water was added. Liquids were removed by vacuum, toluene was added, and then removed by vacuum (to remove traces of pyridine azeotropically). The violet residue was dried under vacuum 1 hour at $60^{\circ}$, then transferred to a Soxhlet thimble, and extracted with water to remove pyridinium chloride. The absorption spectrum of the faintly colored extract solution indicated the presence of protonated porphyrin, so pyridine was added. The excitation spectrum showed nothing, so a few drops of methanol were added to solubilize any precipitate. The excitation spectrum (det. $644 \mathrm{~nm}$ ) then showed peaks at $414,513,548$ and $586 \mathrm{~nm}$, consistent with TCPP. With detection at $691 \mathrm{~nm}$, the 
spectrum was similar (peaks at 415, 513, 549 and $589 \mathrm{~nm}$ ), but weaker, and the Soret band had a significantly broadened base on the long-wavelength side. The emission spectrum (exc. $450 \mathrm{~nm}$ ) showed peaks of nearly equal intensity at 653 and $707 \mathrm{~nm}$, a weak peak at $606 \mathrm{~nm}$, and a peak starting at about $870 \mathrm{~nm}$ and still rising at $900 \mathrm{~nm}$. With excitation at $413 \mathrm{~nm}$, peaks were observed at $605,647,711$ and $815 \mathrm{~nm}$. The absorption spectrum had peaks at $415,519,554,593$ and $649 \mathrm{~nm}$, consistent with TCPP but the Soret had a broad base, like that of TAPP.

The thimble was soaked in $\mathrm{pH} 7$ buffer overnight, and then the buffer solution was poured dropwise through the thimble, followed by $50 \mathrm{~mL}$ of warm water. The thimble was then dried under vacuum at $40^{\circ}$ overnight. The material in the thimble was then chromatographed (silica gel, ethyl acetate eluent) to remove unreacted TAPP. The material left on the column was collected and placed in a Soxhlet thimble and extracted with dichloromethane, and then with ethyl acetate to remove the last traces of TAPP monomer. It was assumed that extraction with stronger solvents would then remove the rest of the porphyrin from the silica gel, but this proved to be untrue. Extraction with $95 \%$ ethanol and with t-butyl methyl ether removed very little material. Pyridine recovered only about $15 \mathrm{mg}$ of solid material. A MALDI mass spectrum of this material indicated the presence of TAPP, TCPP, all four esters of TCPP, a TCPP-TAPP dyad, and a TCPP-TAPP ${ }_{2}$ triad. This mixture could not be reliably separated chromatographically. To remove methyl groups from TCPP, the mixture was treated with $2 \mathrm{mg} \mathrm{NaCN}$ (Mallinckrodt, $30 \mu \mathrm{mol}$ ) and $25 \mathrm{mg}$ $\mathrm{NaI}$ (Mallinckrodt, $167 \mu \mathrm{mol}$ ) in $50 \mathrm{~mL}$ DMF in a $100 \mathrm{~mL}$ round-bottom flask 
containing a stirbar. The solution was brought to reflux, cooled to $120^{\circ}$, and kept at this temperature for 24 hours. Then $10 \mathrm{~mL}$ water were added, and the solution was evaporated to dryness, and the solid residue dried in a vacuum at $40^{\circ}$ overnight. Water was then added, the solution was brought to $\mathrm{pH} 10$ with $\mathrm{NaOH}$, sonicated 20 minutes, and filtered to give a black solid residue and a yellowish filtrate. The filtrate was neutralized with $\mathrm{HCl}$, evaporated to dryness, and chromatographed over cellulose, but resolution was poor. Spectra of the various fractions were found to be contaminated by a nonvolatile fluorescent compound in the AR-grade acetone used to rinse out the cuvettes and the chromatography column. Nevertheless, it was possible to identify at least three different porphyrin components in the mixture removed by base. One had excitation peaks at 429 and $560 \mathrm{~nm}$ and emission peaks at 604 and $655 \mathrm{~nm}$. Addition of base did not change the spectra. The aqueous solution of this material was left unstoppered, and a mold colony grew on it. This and the fact that the excitation spectrum indicated 4-fold symmetry suggested that metallation had occurred. TAPP and TCPP were also present.

Not all of the base-insoluble residue was soluble in DMSO, even after sonication, suggesting some polymeric product. The emission spectrum (exc. 430 $\mathrm{nm}$ ) had a peak at $657 \mathrm{~nm}$ and a shoulder at $710 \mathrm{~nm}$, resembling TCPP but less resolved. With excitation at $450 \mathrm{~nm}$, the emission spectrum showed peaks at $671 \mathrm{~nm}$ and a high shoulder at $691 \mathrm{~nm}$, resembling neither TAPP nor TCPP. The excitation spectrum (det. $635 \mathrm{~nm}$ ) showed a very weak TCPP spectrum, with peaks at 422, 515, 555 and $593 \mathrm{~nm}$. With detection at $655 \mathrm{~nm}$, the excitation spectrum was that of 
TCPP, with peaks at $423,517,554$ and $593 \mathrm{~nm}$.

Due to so little remaining material and the difficulty in separating the mixtures, the experiment was halted.

\section{Synthesis of TCPP-TAPP dyad from TrMCPP}

TCPP $(49.7 \mathrm{mg}, 62.8 \mu \mathrm{mol})$ was converted to TCCPP using the above procedure. The entire TCCPP product was treated with a solution of $8 \mathrm{~mL}$ methanol and $4 \mathrm{~mL}$ pyridine in $15 \mathrm{~mL}$ dichloromethane to methylate all activated carboxyl groups. After stirring the solution overnight, the solvents were evaporated and the violet solid residue was dried under vacuum for 1 hour. It was then chromatographed over silica gel which had been deactivated by slurrying with $15 \%$ water (by weight of dry gel) in acetone, using 3:1 DCM:ethyl acetate to elute the tetramethyl ester (TMCPP), and 3:1 ethyl acetate:DCM to elute the trimethyl ester (TrMCPP).

The TMCPP was partially demethylated by refluxing with $3 \mathrm{mg} \mathrm{NaI}$ and 4 $\mathrm{mg} \mathrm{NaCN}$ in $20 \mathrm{~mL}$ DMF (base hydrolysis using aqueous $\mathrm{KOH}$ proved to be ineffective) for 29 hours, following the reaction with TLC (silica gel, 3:1 DCM:ethyl acetate). After evaporation of the DMF, the solid residue was dissolved in 3:1 DCM:ethyl acetate and chromatographed as above to isolate the TMCPP and TrMCPP. Treatment of the TMCPP with $\mathrm{I}^{-} \mathrm{CN}^{-}$was repeated as above twice more, followed by chromatography, and all TrMCPP fractions were combined.

The TrMCPP was then treated with thionyl chloride (10 drops) in a solution of $0.5 \mathrm{~mL}$ pyridine and $5 \mathrm{~mL}$ toluene. The solution was heated without refluxing 
overnight. The solvent was evaporated, leaving a purple residue. Toluene was added three times, followed by rotary evaporation, to remove pyridine azeotropically. As the pyridine was removed, the $\mathrm{HCl}$ remaining protonated the porphyrin and turned it green. The solid residue was then placed in a vacuum oven at $80^{\circ}$ overnight. Some of it remained greenish, but the reaction was continued anyway. The activated TrMCPP was dissolved in $10 \mathrm{~mL}$ of 2:1 pyridine:toluene, and added dropwise over a period of 30 minutes to a stirred solution of $10.0 \mathrm{mg}$ TAPP (14.8 $\mu \mathrm{mol}$ ) in $15 \mathrm{~mL}$ of $2: 1$ pyridine:toluene in a $50 \mathrm{~mL}$ round-bottom flask. After stirring for 2 hours, the solvents were removed by vacuum. The brownish black residue was dissolved in 3:1 dichloromethane:ethyl acetate and chromatographed over silica gel deactivated with $15 \%$ water by weight of dry gel (see above "Synthesis of TCPP-methyl esters"). The 3:1 DCM:EtOAc eluent brought down a purple band which fluorescence spectroscopy (sample diluted with ethyl acetate) showed was unreacted TrMCPP (exc. $450 \mathrm{~nm}: 651,714 \mathrm{~nm}$, weak spectrum; det. $650 \mathrm{~nm}: 415$, $512,545,589 \mathrm{~nm})$. Methanol: dichloromethane (20:80) brought down nearly all of the rest of the material on the column as a greenish-brown band, leaving only a faint greenish stain throughout the column. This material (diluted with dichloromethane) appeared to be unreacted TAPP (exc. $416 \mathrm{~nm}: 668,722 \mathrm{~nm}$; det. $668 \mathrm{~nm}: 427,522$, $568 \mathrm{~nm})$. 


\section{REFERENCES}

1. De Vos, A. Endoreversible Thermodynamics of Solar Energy Conversion; Oxford University: Oxford, 1992.

2. Möller, H.J. Semiconductors for Solar Cells; Artech House: Boston, 1993.

3. Cook, G. In The Energy Sourcebook; Howes, R; Fainberg, A., Eds. American Institute of Physics: New York, 1991.

4. Zweibel, K. Harnessing Solar Power; Plenum Press: New York, 1990.

5. Hagfeldt, A.; Didriksson, B.; Palmqvist, T.; Lindström, H.; Södergren, S.;

Rensmo, H.; Lindquist, S.-E. Sol. Energy Mat. Sol. Cells 1994, 31, 481-488.

6. Mallouk, T.E. Nature 1991, 353, 698-699.

7. Kay, A.; Grätzel, M. J. Phys. Chem. 1993, 97, 6272-6277.

8. Kavarnos, G.J. Fundamentals of Photoinduced Electron Transfer; VCH: New York, 1993.

9. Scharf, H.-D.; Fleischhauer, J.; Leismann, H.; Ressler, I.; Schleker, W.; Weitz, R. Angew. Chem. Int. Engl. 1979, 18, 652-662.

10. "Standard Tables for Terrestrial Solar Spectral Irradiance at Air Mass 1.5 for a $37^{\circ}$ Tilted Surface". Annual Book of ASTM Standards, Vol. 12.02; American Society for Testing and Materials: Philadelphia, 1993. Designation: E 892-87. There are a number of errors in the table for unnormalized data. Fortunately, since most of the data for this table was taken directly from Bird, R.E.; Hulstrom, R.L.; Lewis, L.J. Solar Energy 1983, 30, 563-573, the correct data could be recovered.

11. Connolly, J.S.; Bolton, J.R. In Photoinduced Electron Transfer, Part D; Fox, M.A.; Chanon, M., Eds. Elsevier: Amsterdam, 1988.

12. Zubay, G. Biochemistry, 3rd ed. Wm. C. Brown: Dubuque, Iowa, 1993. 
13. Nazeeruddin, M.K.; Kay, A.; Rodicio, I.; Humphry-Baker, R.; Müller, E.; Liska, P.; Vlachopoulos, N.; Grätzel, M. J. Am. Chem. Soc., 1993, 115, 6382-63.

14. Bolton, J.R. Science 1978, 202, 705-711.

15. Ross, R.T.; Hsiao, T.-L. J. Appl. Phys. 1977, 48, 4783-4785.

16. Bolton, J.R.; Hall, D.O. Ann. Rev. Energy 1979, 4, 353-401.

17. Pirt, S.J. New Phytol. 1986, 102, 3-37.

18. Duysens, L.N.M. Brookhaven Symposium of Biology 1958, 11, 10-25.

19. Spanner, D.C. Introduction to Thermodynamics; Academic Press: London, 1964.

20. Henry, C.H. J. Appl. Phys. 1980, 51(8), 4494-4500.

21. Ross, R.T.; Collins, J.M. J. Appl. Phys. 1980, 51, 4504-4507.

22. Bolton, J.R.; Hall, D.O. Photochem. Photobiol. 1991, 53, 545-548.

23. Björkmann, O.; Demmig, B. Planta, 1987, 170, 489-504.

24. Walker, D. New Phytol. 1992, 121, 325-345.

25. Hill, R.; Bendall, F. Nature 1960, 186, 136-137.

26. De Vos, A. Sol. Energy Mat. Sol. Cells 1993, 31, 75-93.

27. Pitchford, P.; Jones, J.; Glenn, B.; Cook, G.; Billman, L.; Adcock, R. Photovoltaic Fundamentals; National Renewable Energy Lab, September 1991. DOE/CH/10093-117.

28. Araújo, G.L.; Martí, A. IEEE Trans. Elec. Devices 1990, 37, 1402-1405.

29. Pool, R. Science, 1988, 241, 900-901.

30. Wasielewski, M.R. Chem. Rev. 1992, 92, 435-461.

31. DeGraziano, J.M.; Liddell, P.A.; Leggett, L.; Moore, A.L.; Moore, T.A.; Gust, D. J. Phys. Chem. 1994, 98, 1758-1761. 
32. Gust, D.; Moore, T.A.; Moore, A.L.; Gao, F.; Luttrull, D.; DeGraziano, J.M.; Ma, X.C.; Makings, L.R.; Lee, S.-J., et al. J. Am. Chem. Soc. 1991, 113, 3638-3649.

33. Vergeldt, F.J.; Koehorst, R.B.M.; Schaafsma, T.J.; Lambry, J.-C.; Martin, J.-L.; Johnson D.G.; Wasielewski, M.R. Chem. Phys. Lett. 1991, 182, 107-113.

34. Gust, D.; Moore, T.A.; Moore, A.L.; Kang, H.K.; DeGraziano, J.M.; Liddell, P.A.; Seely, G.R. J. Phys. Chem. 1993, 97, 13637-13642.

35. Wasielewski, M.R.; Johnson, D.G.; Niemczyk, M.P.; Gaines, G.L., III; O'Neil, M.P.; Svec, W.A. J. Am. Chem. Soc. 1990, 112, 6482-6488.

36. Sakata, Y.; Tsue, H.; O'Neil, M.P.; Wiederrecht, G.P.; Wasielewski, M.R. J. Am. Chem. Soc. 1994, 116, 6904-6909.

37. Dynamics and Mechanisms of Photoinduced Electron Transfer and Related Phenomena; Mataga, N., Okada, T., Masuhara, H., Eds. North-Holland: Amsterdam, 1992.

38. Hung, S.-C.; Macpherson, A.N.; Lin, S.; Liddell, P.A.; Seely, G.R.; Moore, A.L.; Moore, T.A.; Gust, D. J. Am. Chem. Soc. 1995, 117, 1657-1658.

39. Gust, D.; Moore, T.A.; Moore, A.L. Acc. Chem. Res. 1993, 26, 198-205.

40. Ohkohchi, M.; Takahashi, A.; Mataga, N.; Okada, T.; Osuka, A.; Yamada, H.; Maruyama, K. J. Am. Chem. Soc. 1993, 115, 12137-12143.

41. Sessler, J.L.; Capuano, V.L.; Harriman, A. J. Am. Chem. Soc. 1993, 115, 4618-4628.

42. Grätzel, M. Heterogeneous Photochemical Electron Transfer; CRC: Boca Raton, Florida, 1989.

43. Kalyanasundaram, K.; Vlachopoulos, N.; Krishnan, V.; Monnier, A.; Grätzel, M. J. Phys. Chem. 1987, 91, 2342-2347.

44. Dabestani, R.; Bard, A.J.; Campion, A.; Fox, M.A.; Mallouk, T.E.; Webber, S.E.; White, J.M. J. Phys. Chem. 1988, 92, 1872-1878.

45. O'Regan, B.; Moser, J.; Anderson, M.; Grätzel, M. J. Phys. Chem. 1990, 94, 8720-8726.

46. Kay, A.; Humphry-Baker, R.; Grätzel, M. J. Phys. Chem. 1994, 98, 952-959. 
47. Grätzel, M. In Electrochemistry in Colloids and Dispersions; Mackay, R.A.; Texter, J., Eds. VCH: New York, 1992; 357-374.

48. Södergren, S.; Hagfeldt, A.; Olsson, J.; Lindquist, S.-E. J. Phys. Chem. 1994, $98,5552-5556$.

49. Howe, R.F.; Grätzel, M. J. Phys. Chem. 1985, 89, 4495-4499.

50. Moser, J.; Punchihewa, S.; Infelta, P.P.; Grätzel, M. Langmuir 1991, 7, 30123018.

51. Hagfeldt, A.; Lindquist, S.-E.; Grätzel, M. Sol. Energy Mat. Sol. Cells 1994, 32, 245-257.

52. Sclafani, A.; Palmisano, L.; Schiavello, M. J. Phys. Chem. 1990, 94, 829-832.

53. Péchy, P.; Rotzinger, F.P.; Nazeeruddin, M.K.; Kohle, O.; Zakeeruddin, S.M.; Humphry-Baker, R.; Grätzel, M. J. Chem. Soc., Chem. Commun. 1995, 1, 6566.

54. Tunesi, S.; Anderson, M.A. Langmuir 1992, 8, 487-495.

55. O'Regan, B.; Grätzel, M. Nature 1991, 353, 737-740.

56. Grätzel, M.; Liska, P. U.S. Patent No. 5,084,365, 1992.

57. Smestad, G. Sol. Energy Mat. Sol. Cells 1994, 32, 273-288.

58. Knödler, R.; Sopka, J.; Harbach, F.; Grünling, H.W. Sol. Energy Mat. Sol. Cells 1993, 30, 277-281.

59. Smestad, G.; Bignozzi, C.; Argazzi, R. Sol. Energy Mat. Sol. Cells 1994, 32, 259-272.

60. Gregg, B.A.; Kim, Y.I. J. Phys. Chem. 1994, 98, 2412-2417.

61. Wamser, C.C. unpublished results.

62. Ransdell, R.A.; Wamser, C.C. J. Phys. Chem. 1992, 96, 10572-10575.

63. Gust, D.; Moore, T.A.; Moore, A.L.; Leggett, L.; Lin, S.; DeGraziano, J.M.; Hermant, R.M.; Nicodem, D.; Craig, P.; Seely, G.R.; Nieman, R.A. J. Phys. Chem. 1993, 97, 7926-7931. 
64. Klausner, Y.S.; Bodansky, M. Synthesis 1972, 453-463.

65. Williams, A.; Ibrahim, I.T. Chem. Rev. 1981, 81, 589-636.

66. Neises, B.; Steglich, W. Angew. Chem. Int Ed. Engl. 1978, 17, 522-523.

67. $\mathrm{Li}, \mathrm{W}$. unpublished results.

68. Carey, F.A.; Sundberg, R.J. Advanced Organic Chemistry, Part A, 3rd ed. Plenum: New York, 1990.

69. Pouchert, C.J.; Behnke, J. The Aldrich Library of ${ }^{13} \mathrm{C}$ and ${ }^{1} H$ FT NMR Spectra, Edition 1, vol. I; Aldrich Chemical: Milwaukee, 1993, pp. 1339B and 1412C.

70. Van Regenmortel, M.H.V. Synthetic Polypeptides as Antigens; Elsevier: Amsterdam, 1988.

71. Stewart, J.M.; Young, J.D. Solid Phase Peptide Synthesis. 2nd ed. Pierce Chemical Co.: Rockford, IL, 1984.

72. Barlos, K.; Chatzi, O.; Gatos, D.; Stavropoulos, G. Int. J. Peptide Protein Res. 1991, 37, 513-520.

73. Lee, J.B. J. Am. Chem. Soc. 1966, 88, 3440-3441.

74. Barstow, L.E.; Hruby, V.J. J. Org. Chem. 1971, 36, 1305-1306.

75. McMurry, J.E., Wong, G.B. Synth. Commun. 1972, 2, 389-394.

76. Ojadi, E.C.A.; Linschitz, H.; Gouterman, M.; Walter, R.I.; Lindsey, J.S.; Wagner, R.W.; Droupadi, P.R.; Wang, W. J. Phys. Chem. 1993, 97, 1319213197.

77. Walter, R.I.; Ojadi, E.C.A.; Linschitz, H. J. Phys. Chem. 1993, 97, 13308-13312.

78. Bard, R., unpublished results.

79. The author is grateful to J. Donovan at EM Separations for this helpful information.

80. Clarke, S.E. Portland State University PhD Dissertation, May 1990.

81. Dictionary of Organic Compounds, 5th ed.; Buckingham, J., Ed. Chapman and Hall: London, 1982. 
82. Beilsteins Handbuch der Organischen Chemie. Springer-Verlag: Berlin, 12 III 595.

83. The Merck Index, 11 th ed. Budavari, S., et al., Eds. Merck: Rahway, New Jersey, 1989.

84. Dolphin, D. J. Chromatog. Library 1983, 22B, 377-406.

85. Nargiello, M.; Herz, T. In Photocatalytic Purification and Treatment of Water and Air; Ollis, D.F.; Al-Ekabi, H., Eds. Elsevier: Amsterdam, 1993. 
APPENDIX FIGURES 


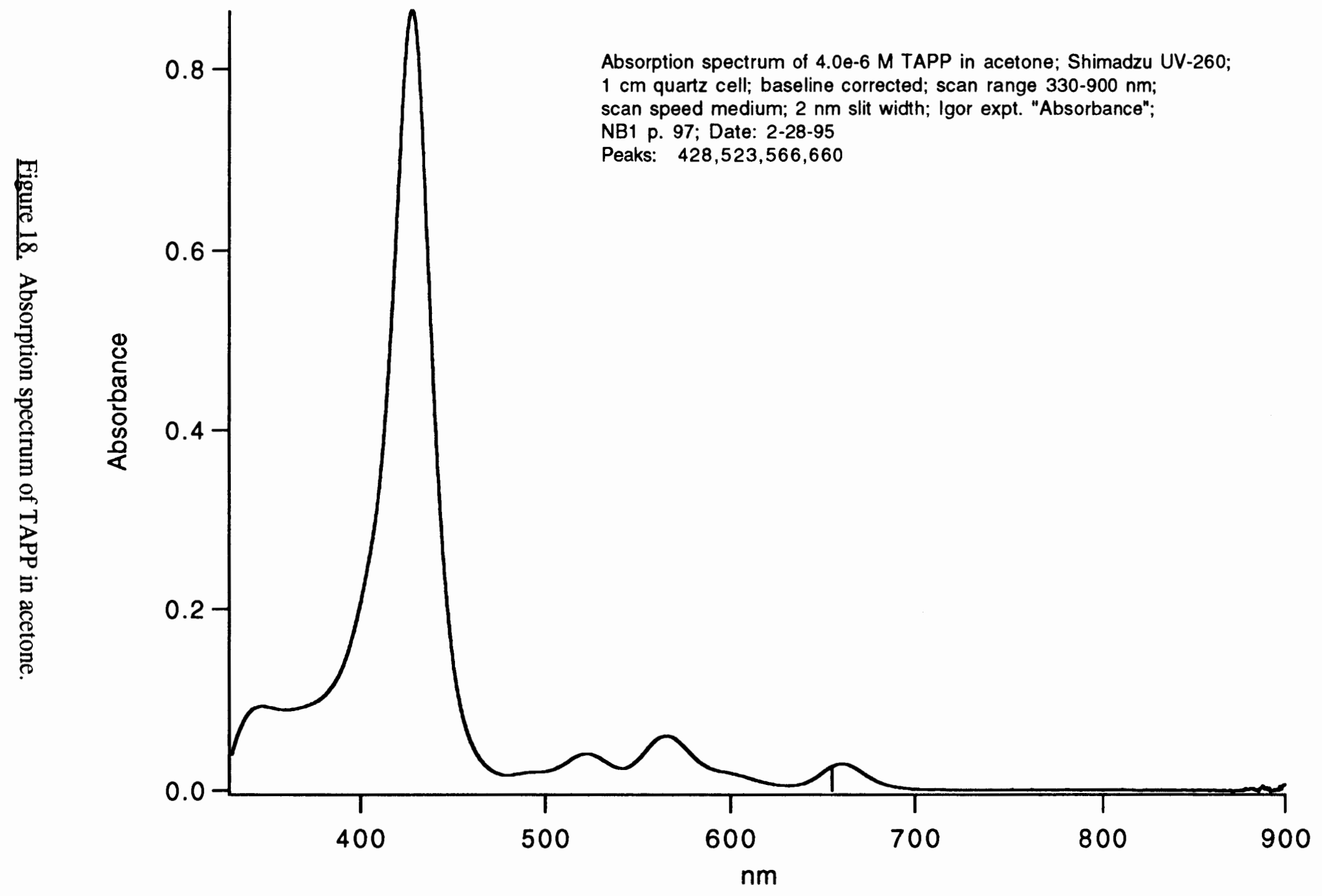




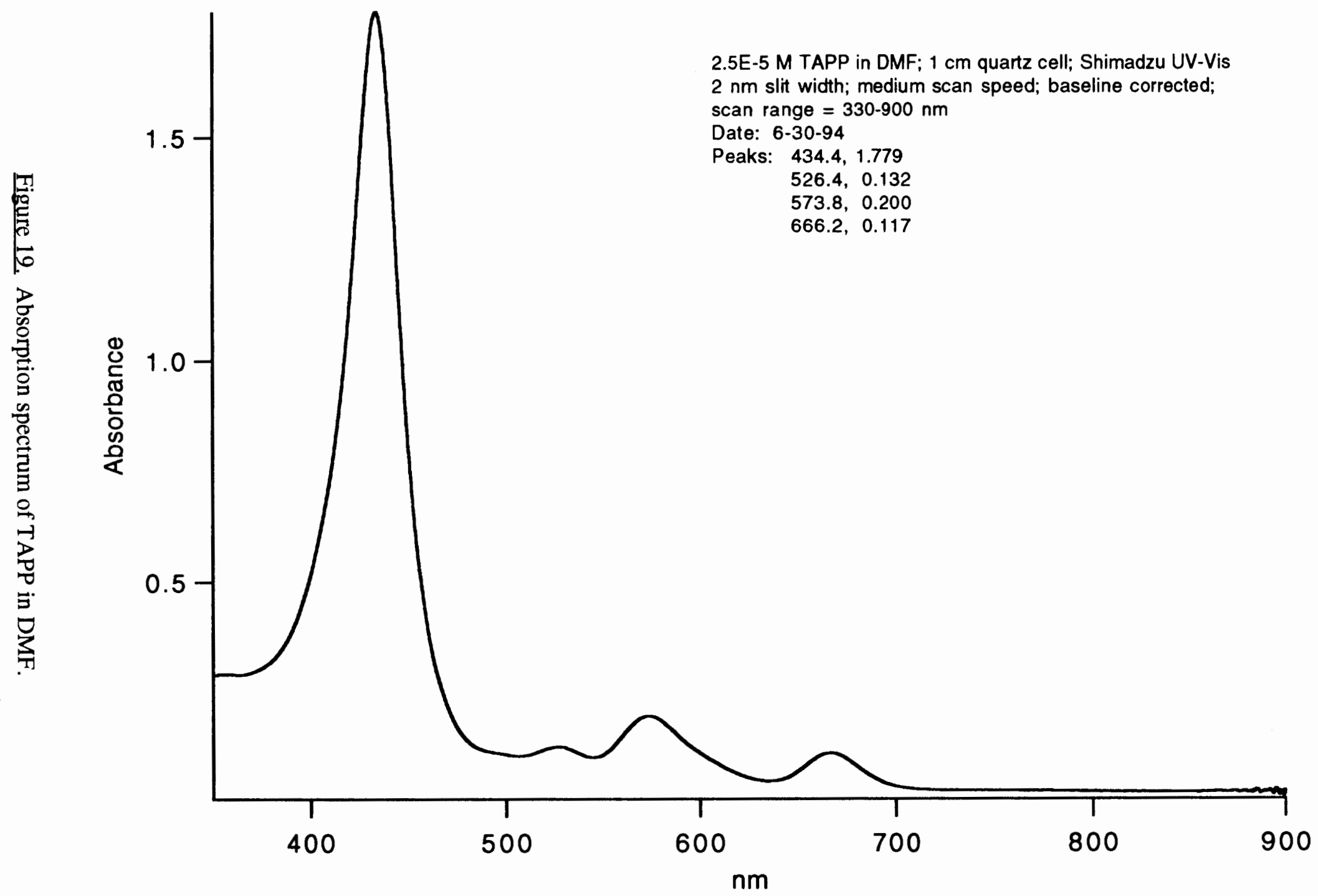




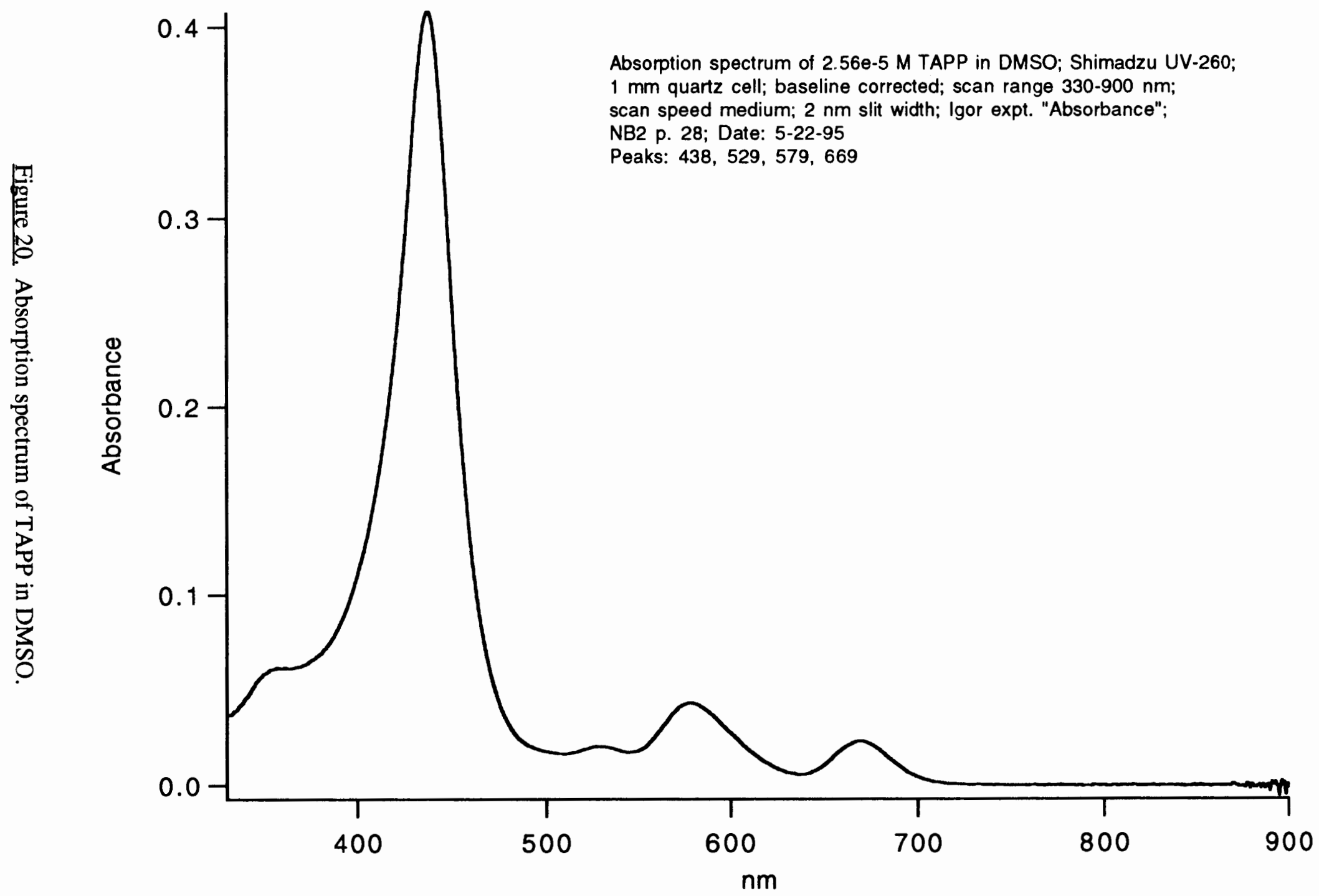




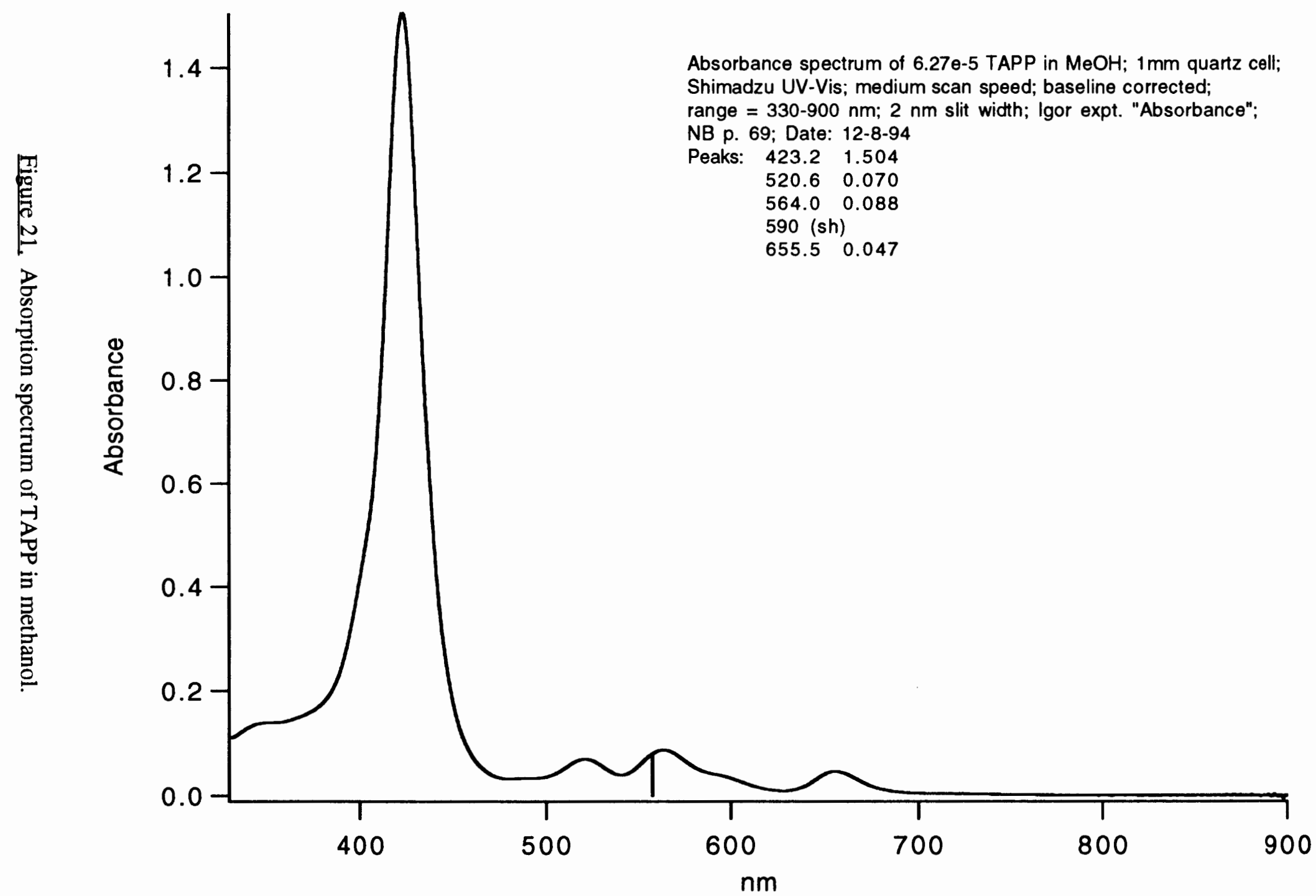




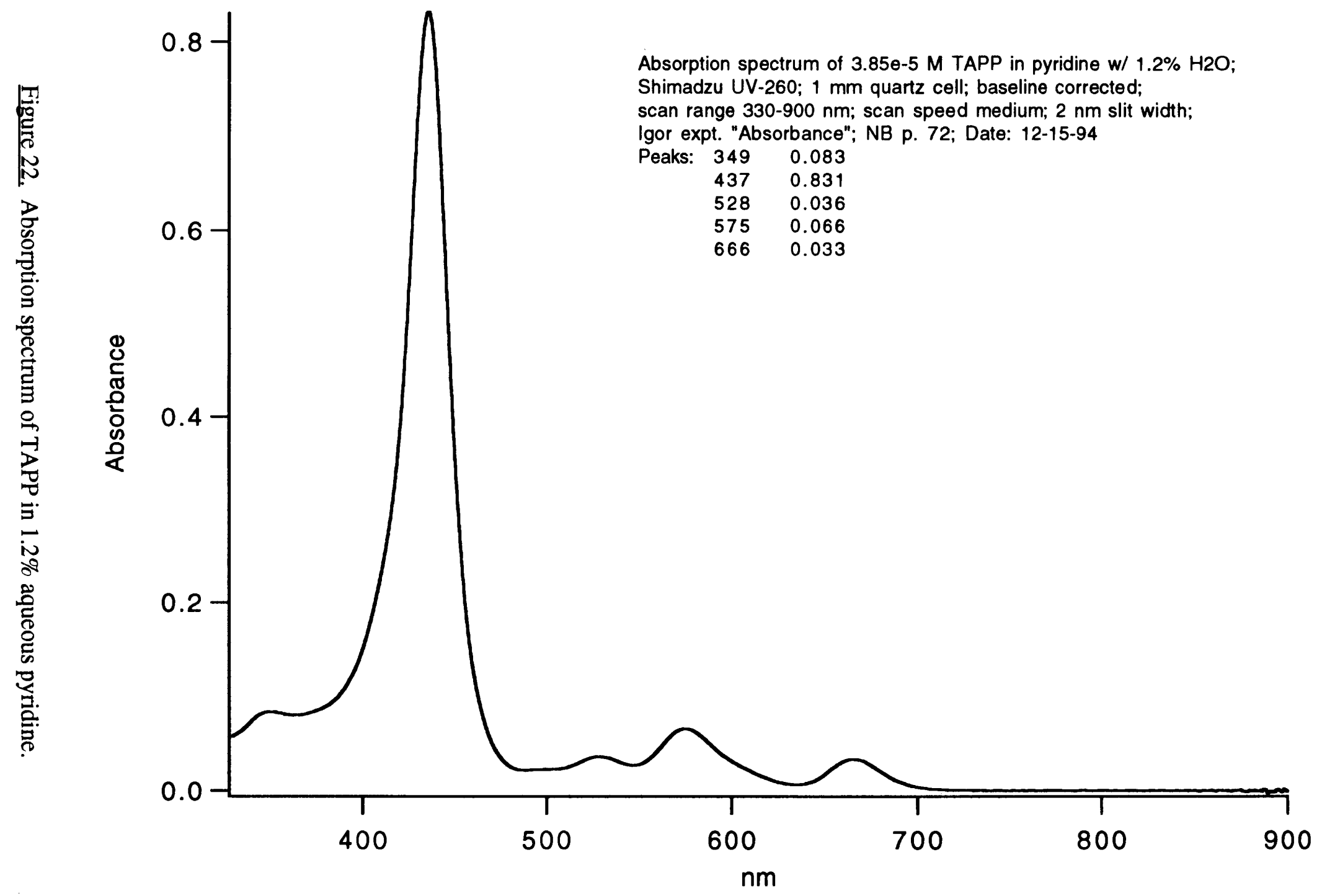




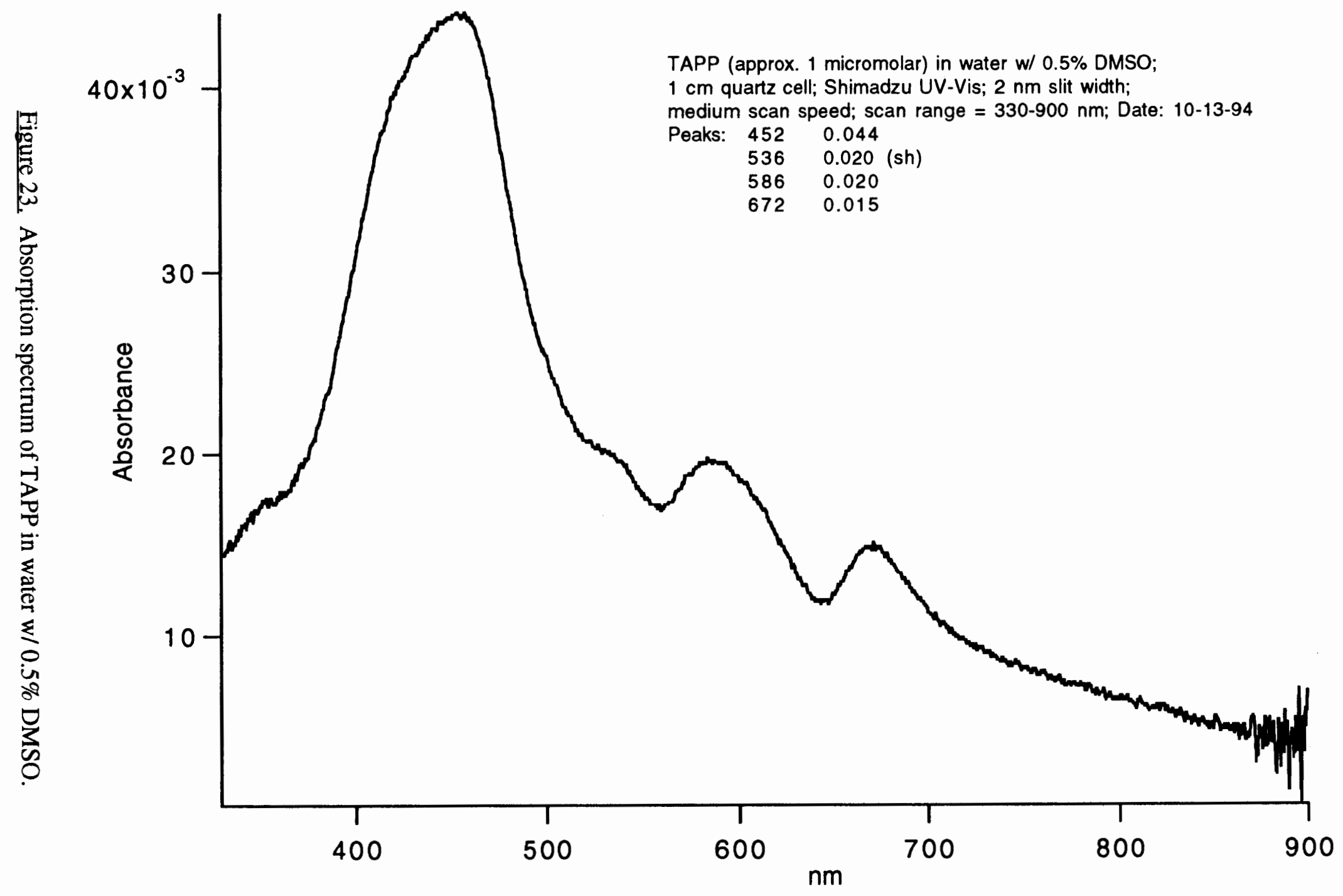




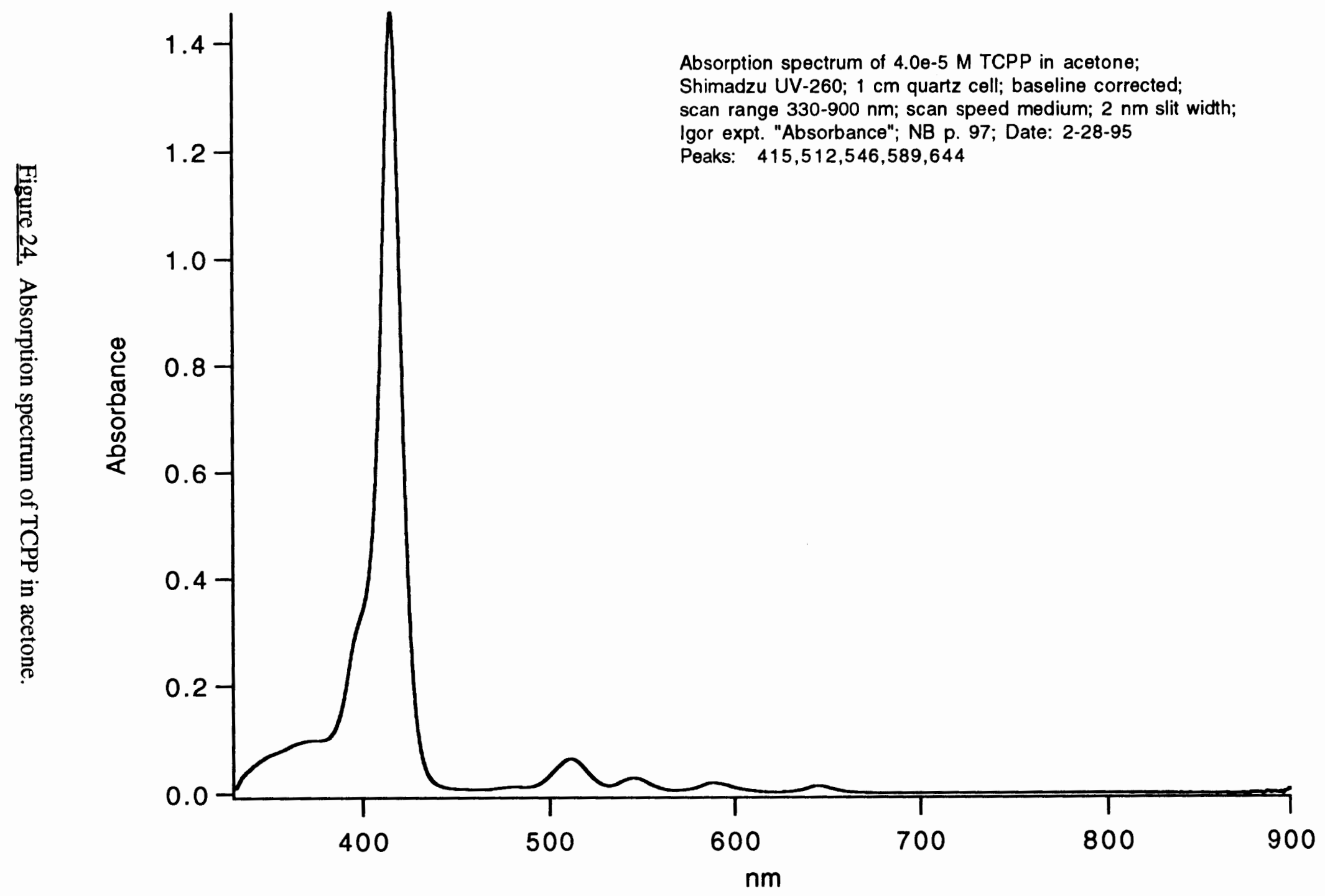




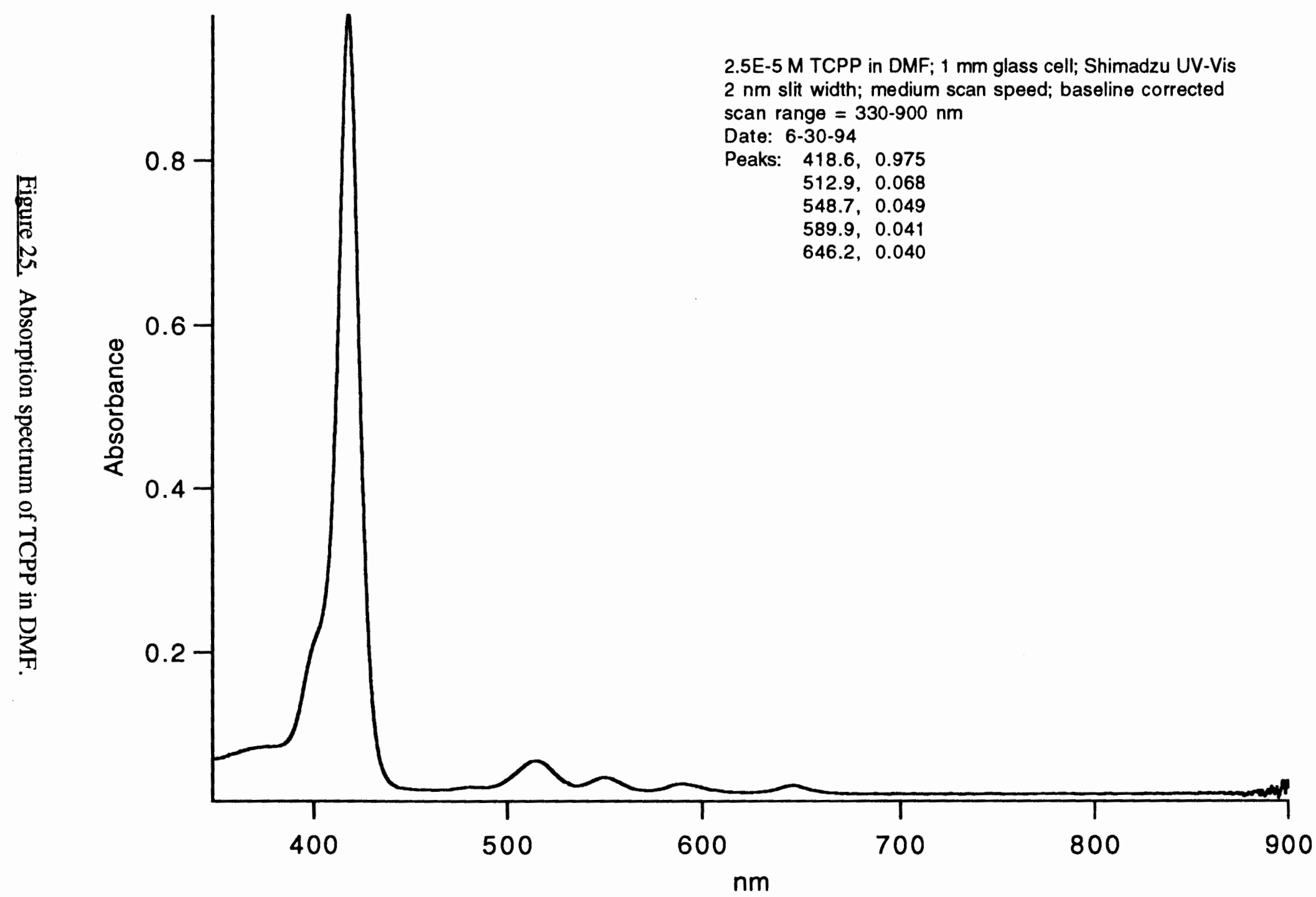




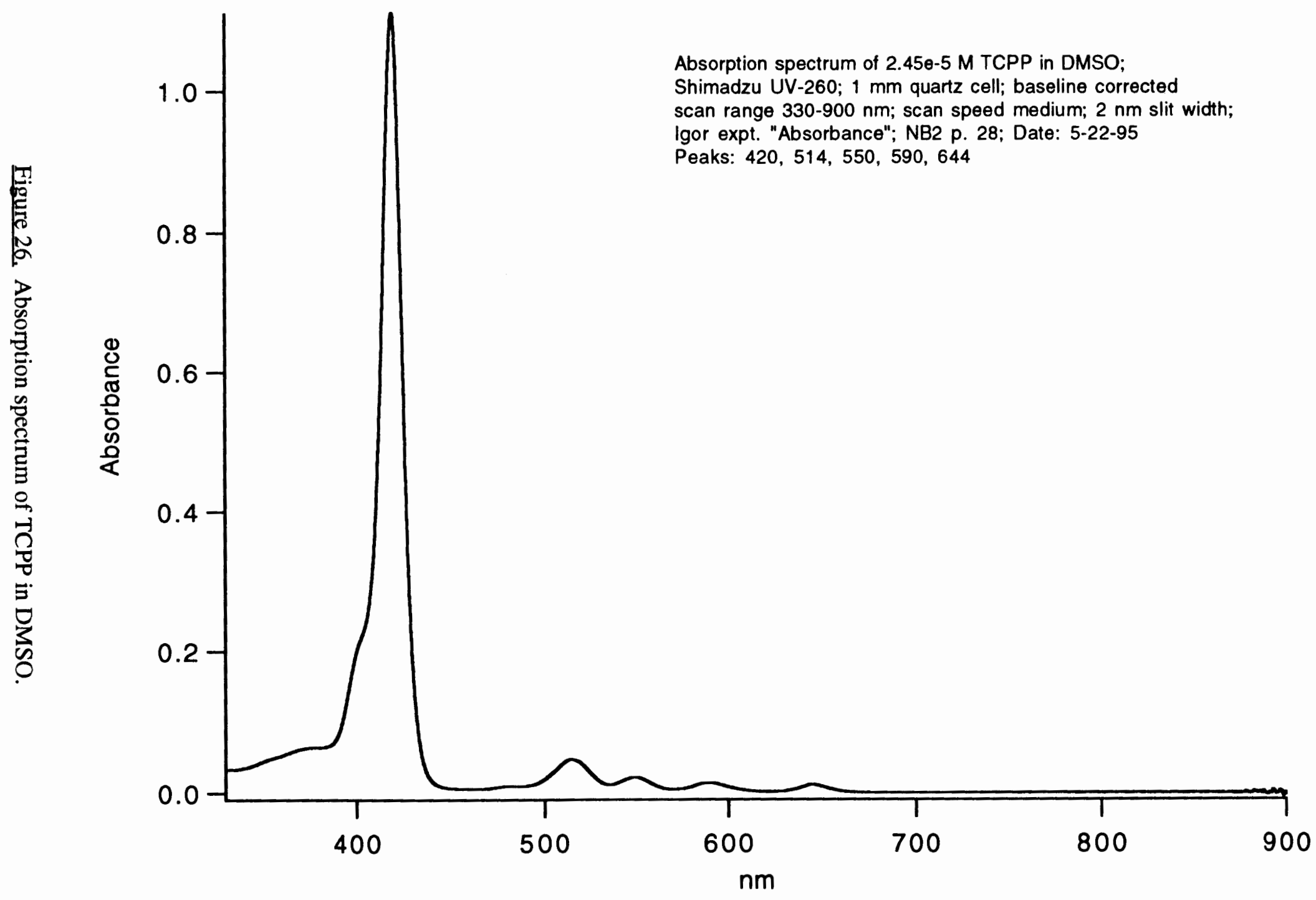




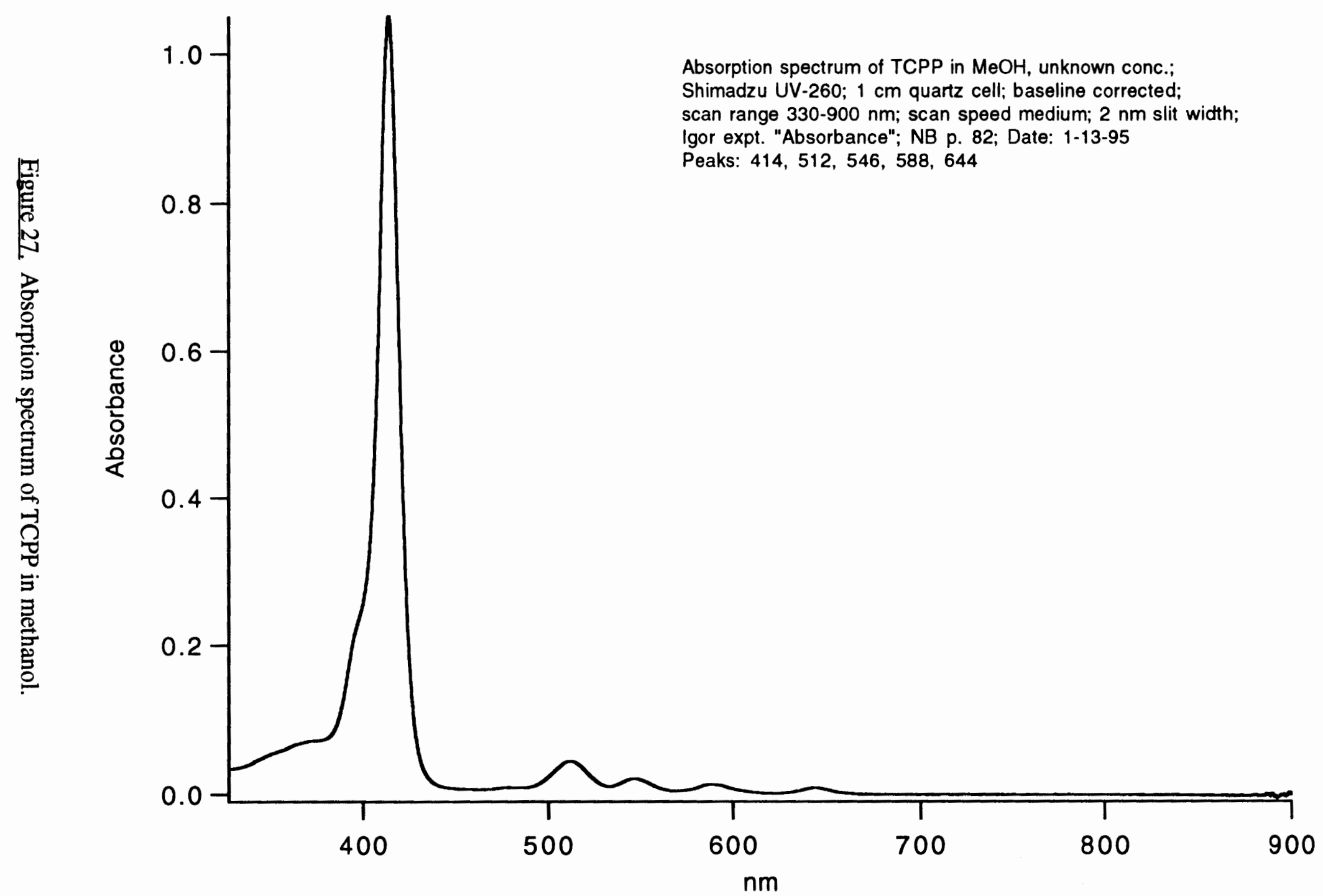




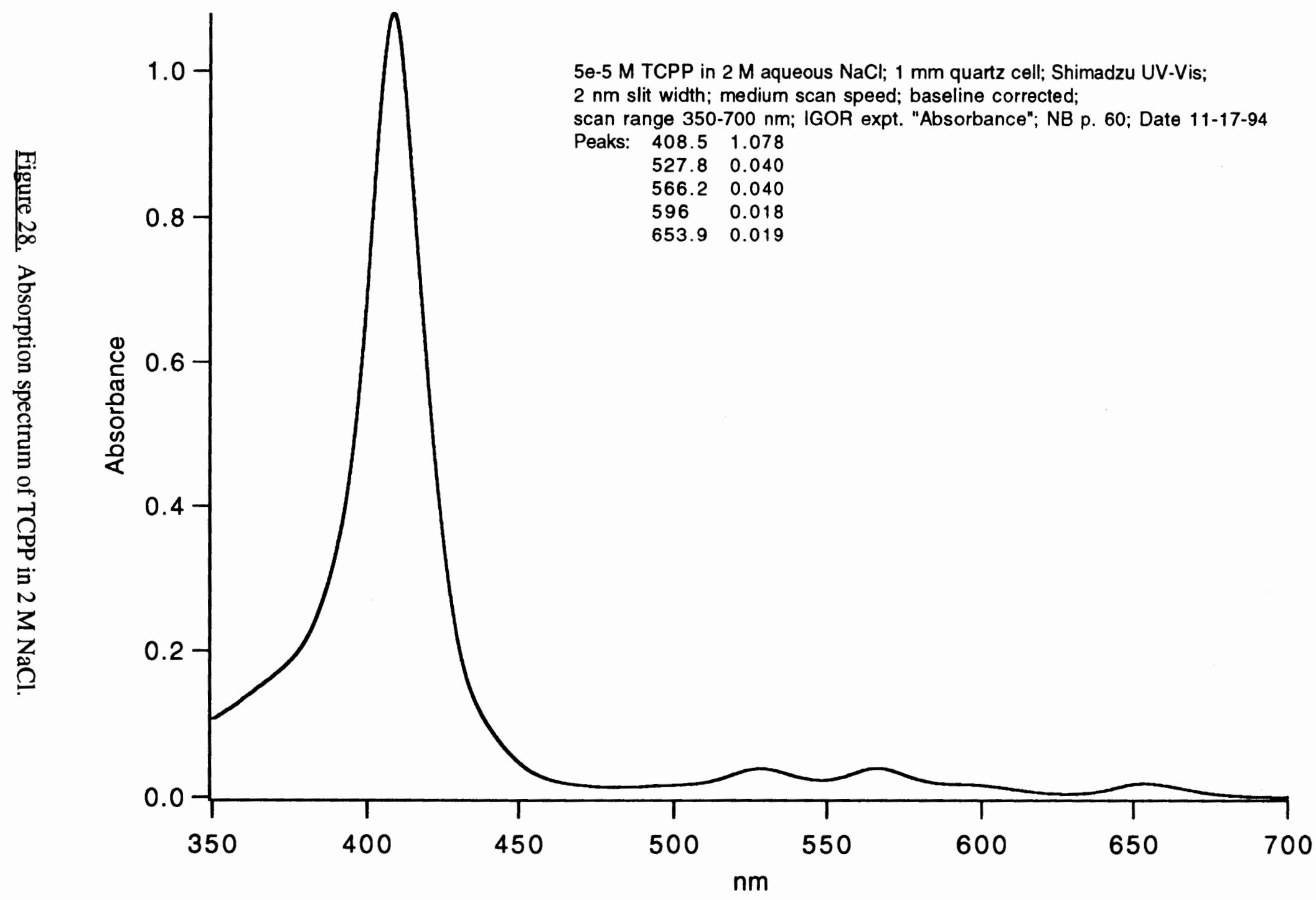




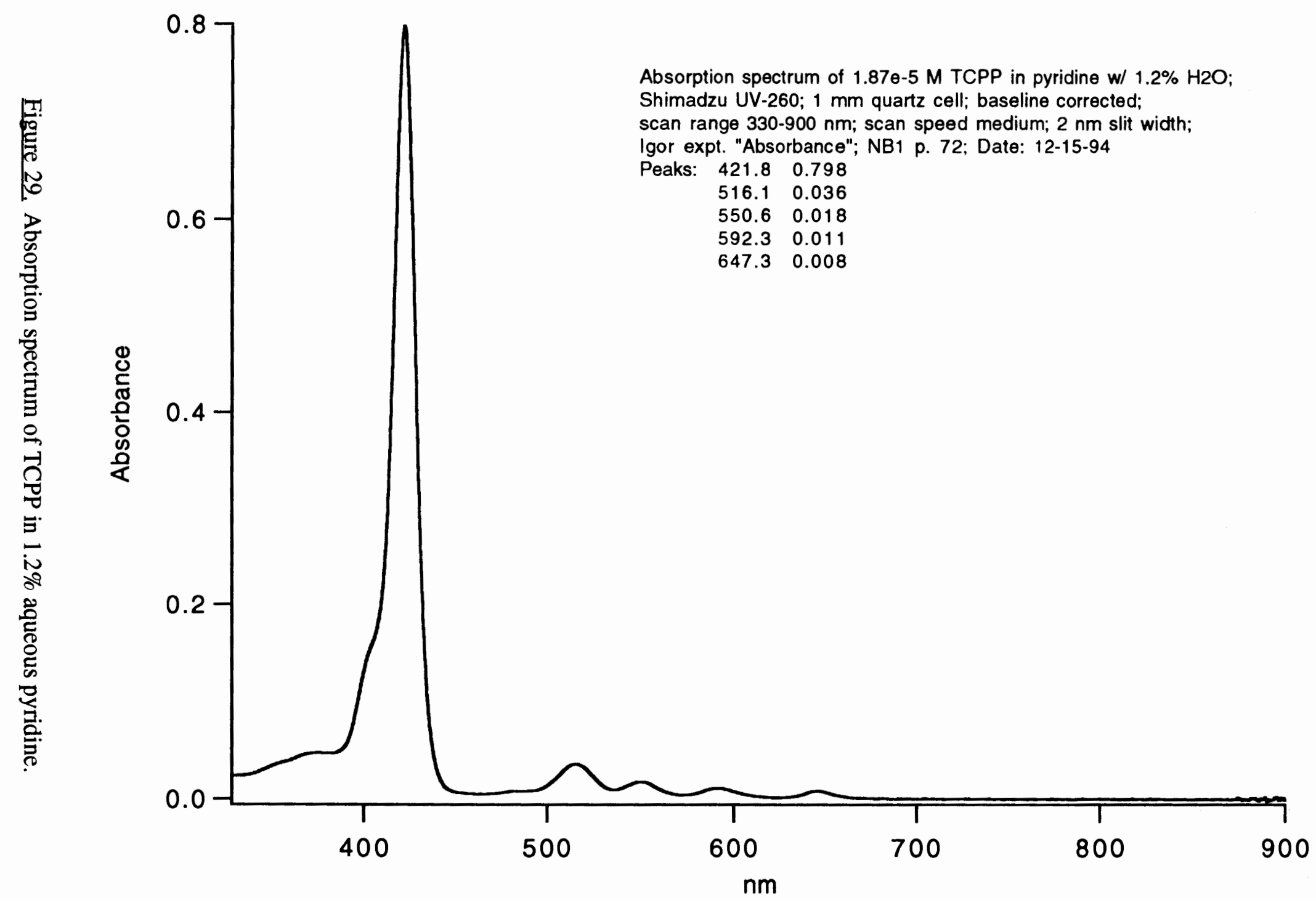




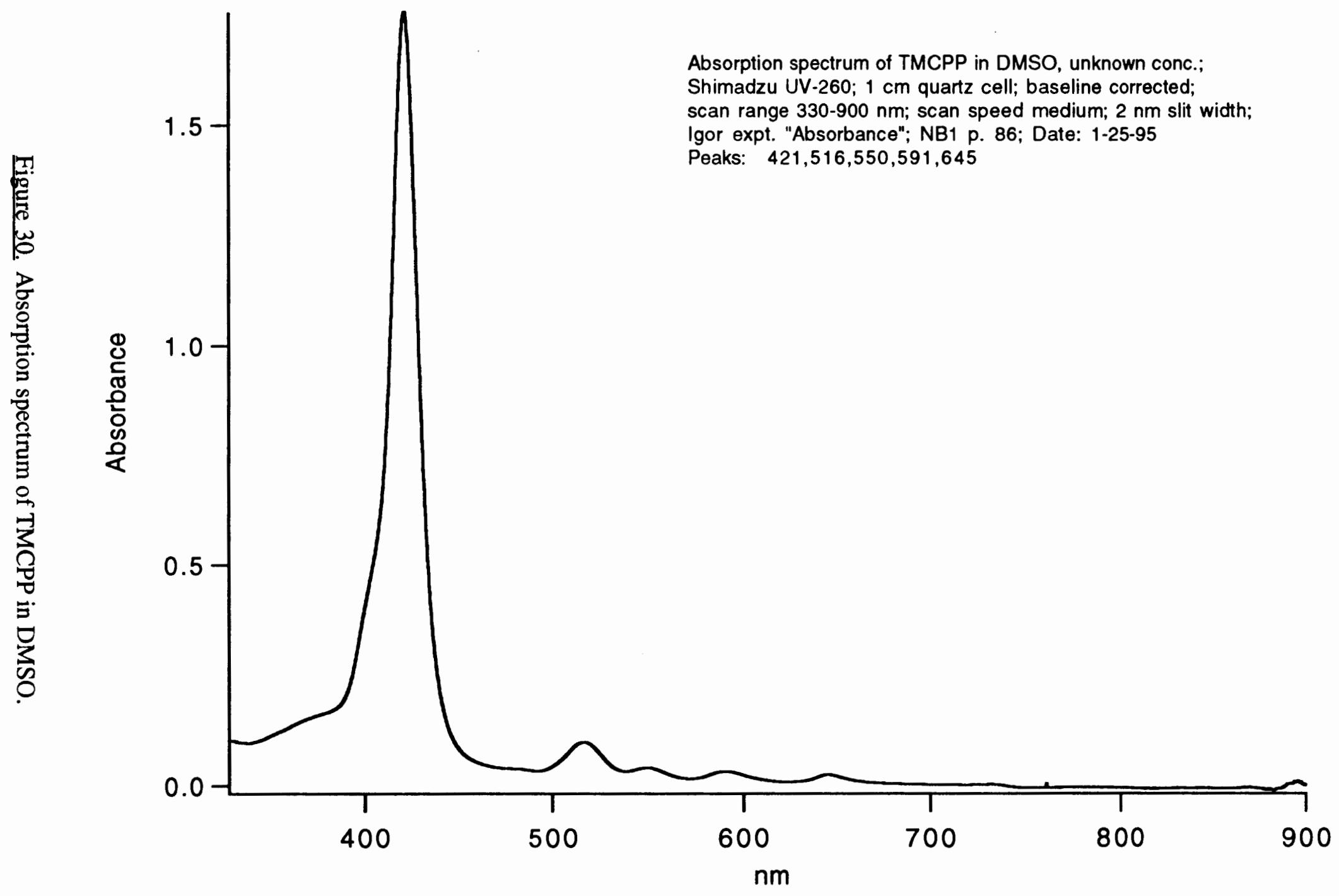




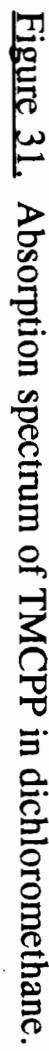

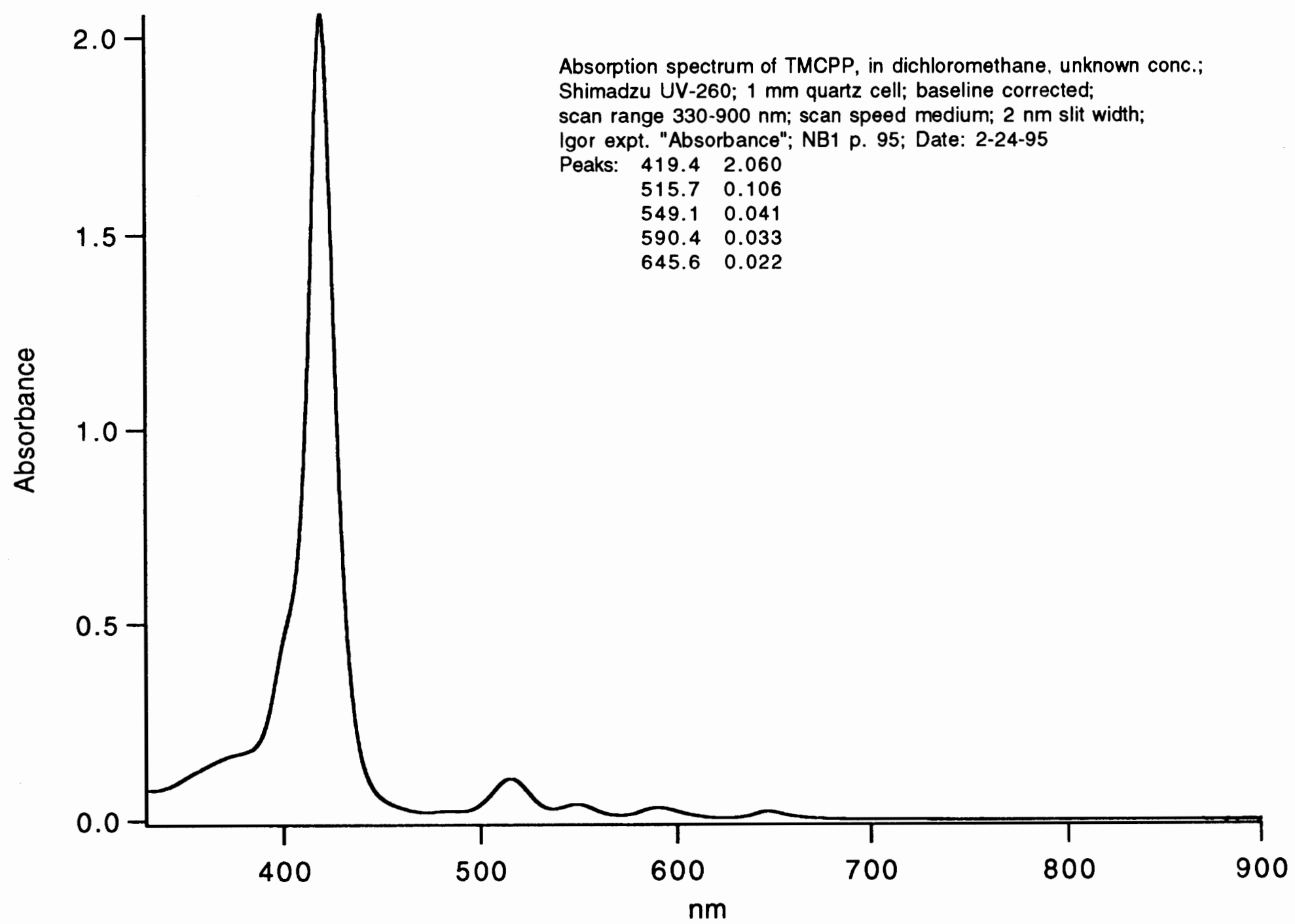




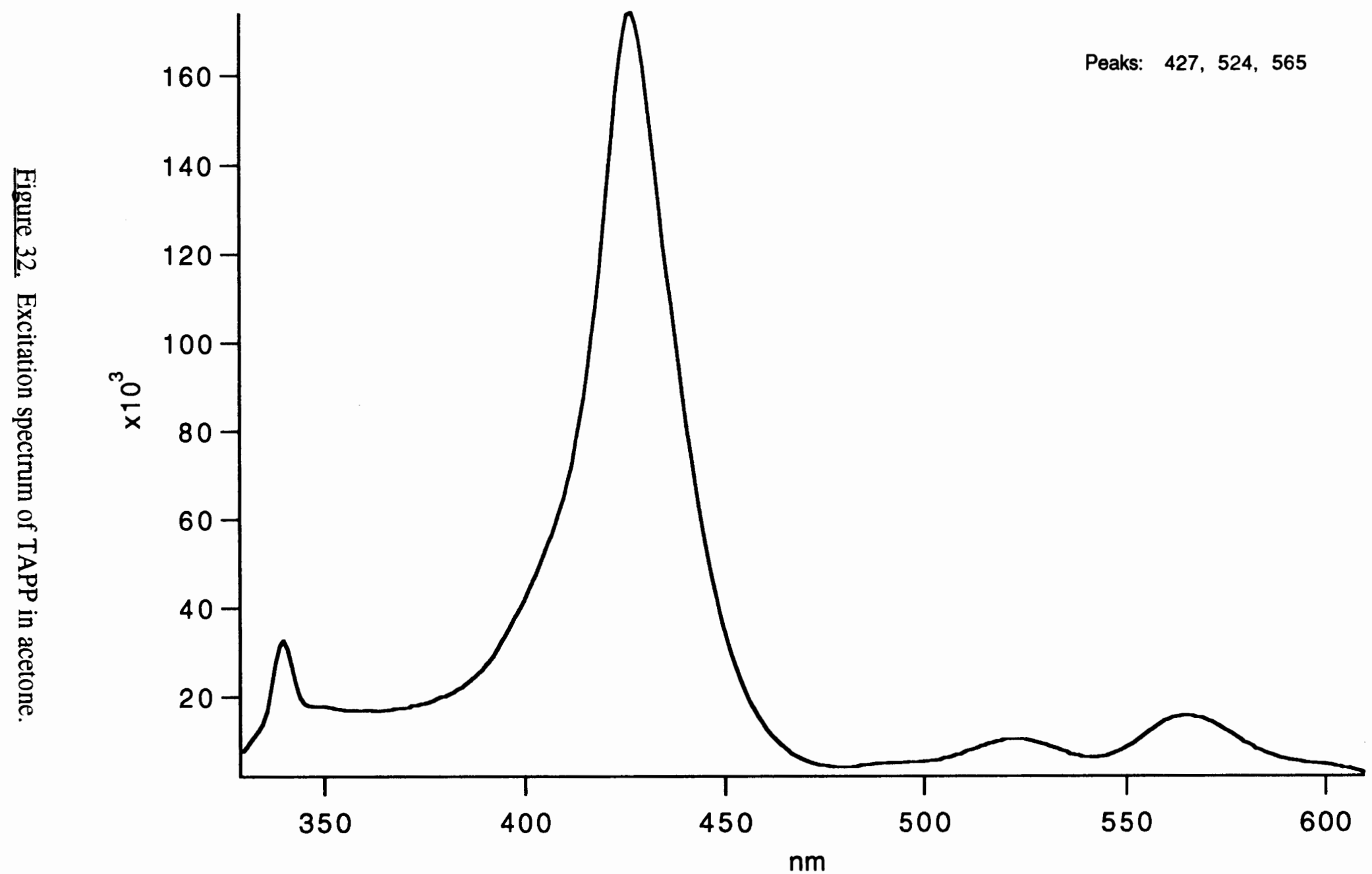

Excitation spectrum of 1e-6 M TAPP in acetone; detection at $682 \mathrm{~nm}$; SPEX Fluorolog;

range = 330-610; $1 \mathrm{~nm}$ incr.; 1 sec. integ. time; PMT voltages = 990/580; acq. mode $=7$ (S1/S2);

exc. slits $=0.5 \mathrm{~mm}$; em. slits $=5.0 \mathrm{~mm} ; 1 \mathrm{~cm}$ quartz cell; right angle detection; corrected for source variation;

lgor expt. "Excitation"; NB p. 95; Date: 2-11-95 


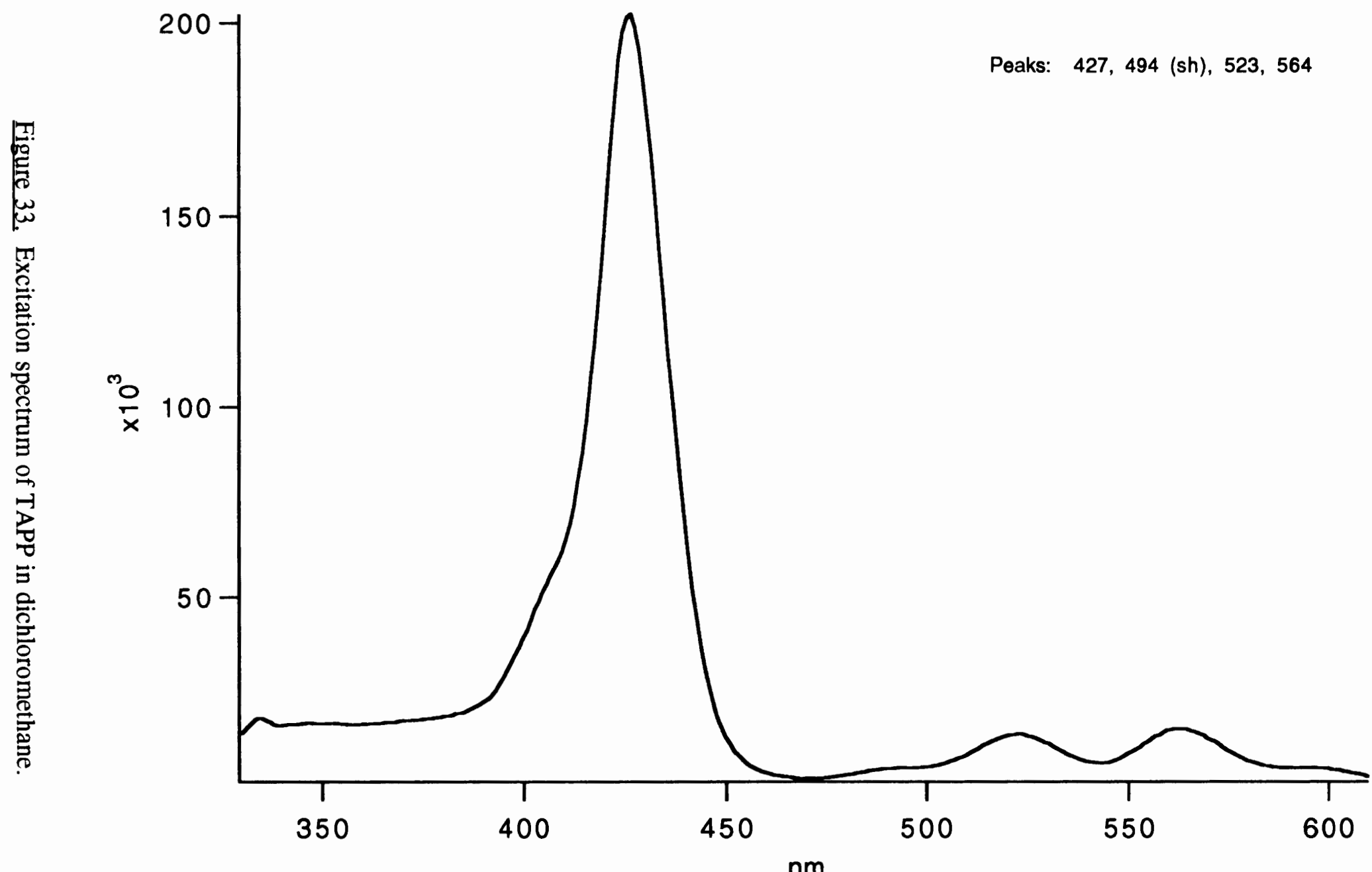

$\mathrm{nm}$

Excitation spectrum of TAPP in $\mathrm{CH} 2 \mathrm{Cl} 2$, unknown conc.; detection at $670 \mathrm{~nm}$; SPEX Fluorolog; range = 330-610; $1 \mathrm{~nm}$ incr.; 1 sec. integ. time; PMT voltages = 950/580; acq. $\operatorname{mode}=7$ (S1/S2);

exc. slits $=0.5 \mathrm{~mm}$; em. slits $=5.0 \mathrm{~mm} ; 1 \mathrm{~cm}$ quartz cell; right angle detection; corrected for source variation; lgor expt. "Excitation"; NB1 p. 97; Date: 2-24-95 


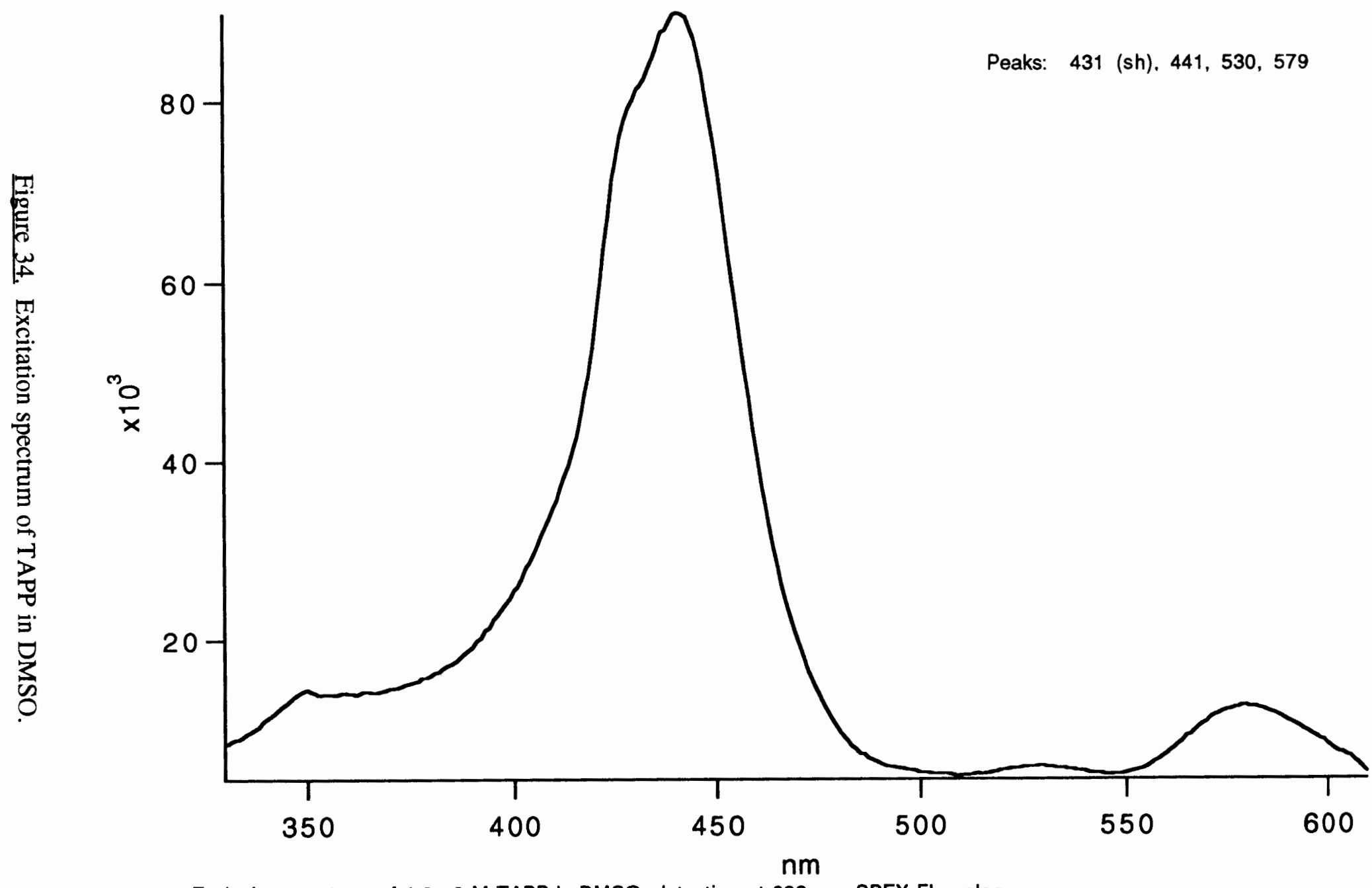

Excitation spectrum of 1.00-6 M TAPP in DMSO; detection at $699 \mathrm{~nm}$; SPEX Fluorolog;

range = 330-610; $1 \mathrm{~nm}$ incr.; 1 sec. integ. time; PMT voltages = 990/580; acq. $\operatorname{mod\theta }=7$ (S1/S2);

exc. slits $=0.5 \mathrm{~mm}$; em. slits $=5.0 \mathrm{~mm} ; 1 \mathrm{~cm}$ quartz cell; right angle detection; corrected for source variation; Igor expt. "Excitation"; NB2 p. 28; Date: 5-22-95 


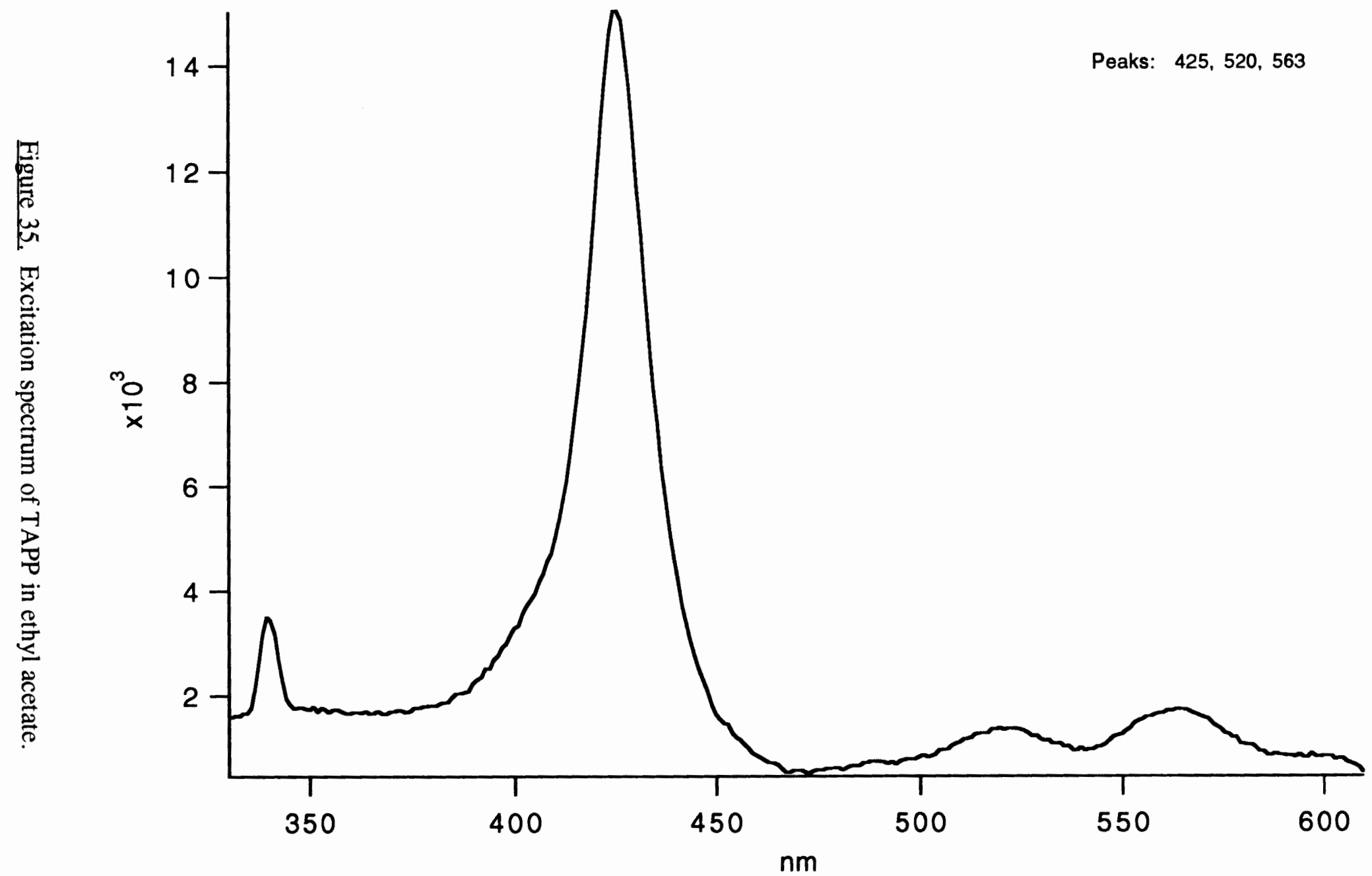

Excitation spectrum of 1e-6 M TAPP in EIOAc; detection at $682 \mathrm{~nm}$; SPEX Fluorolog;

range $=330-610 ; 1 \mathrm{~nm}$ incr.; 1 sec. integ. time; PMT voltages $=990 / 580 ;$ acq. mode $=7$ (S1/S2);

exc. slits $=0.5 \mathrm{~mm}$; em. slits $=5.0 \mathrm{~mm} ; 1 \mathrm{~cm}$ quartz cell; right angle detection; corrected for source variation;

Igor expt. "Excitation"; NB p. 94; Date: 2-13-95 


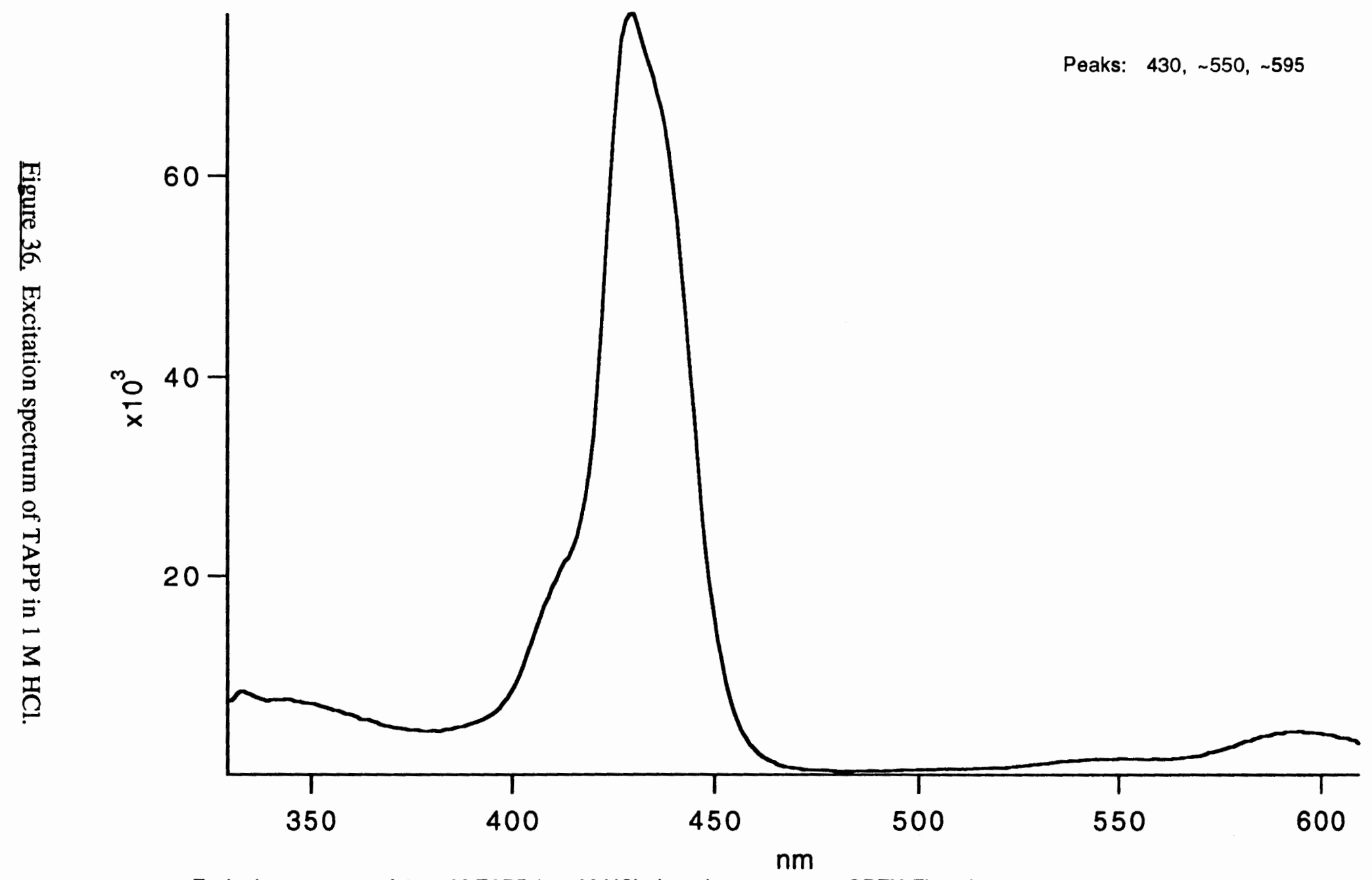

Excitation spectrum of $2 \theta-6 \mathrm{M}$ TAPP in $1 \mathrm{M} \mathrm{HCl}$; detection at $668 \mathrm{~nm}$; SPEX Fluorolog;

range = 330-610; $1 \mathrm{~nm}$ incr.; 1 sec. integ. time; PMT voltages $=990 / 580 ;$ acq. mode $=7$ (S1/S2);

exc. slits $=0.5 \mathrm{~mm} ;$ em. slits $=5.0 \mathrm{~mm} ; 1 \mathrm{~cm}$ quartz cell; right angle detection; corrected for source variation;

Igor expt. "Excitation"; NB2 p. 12; Date: 4-13-95 


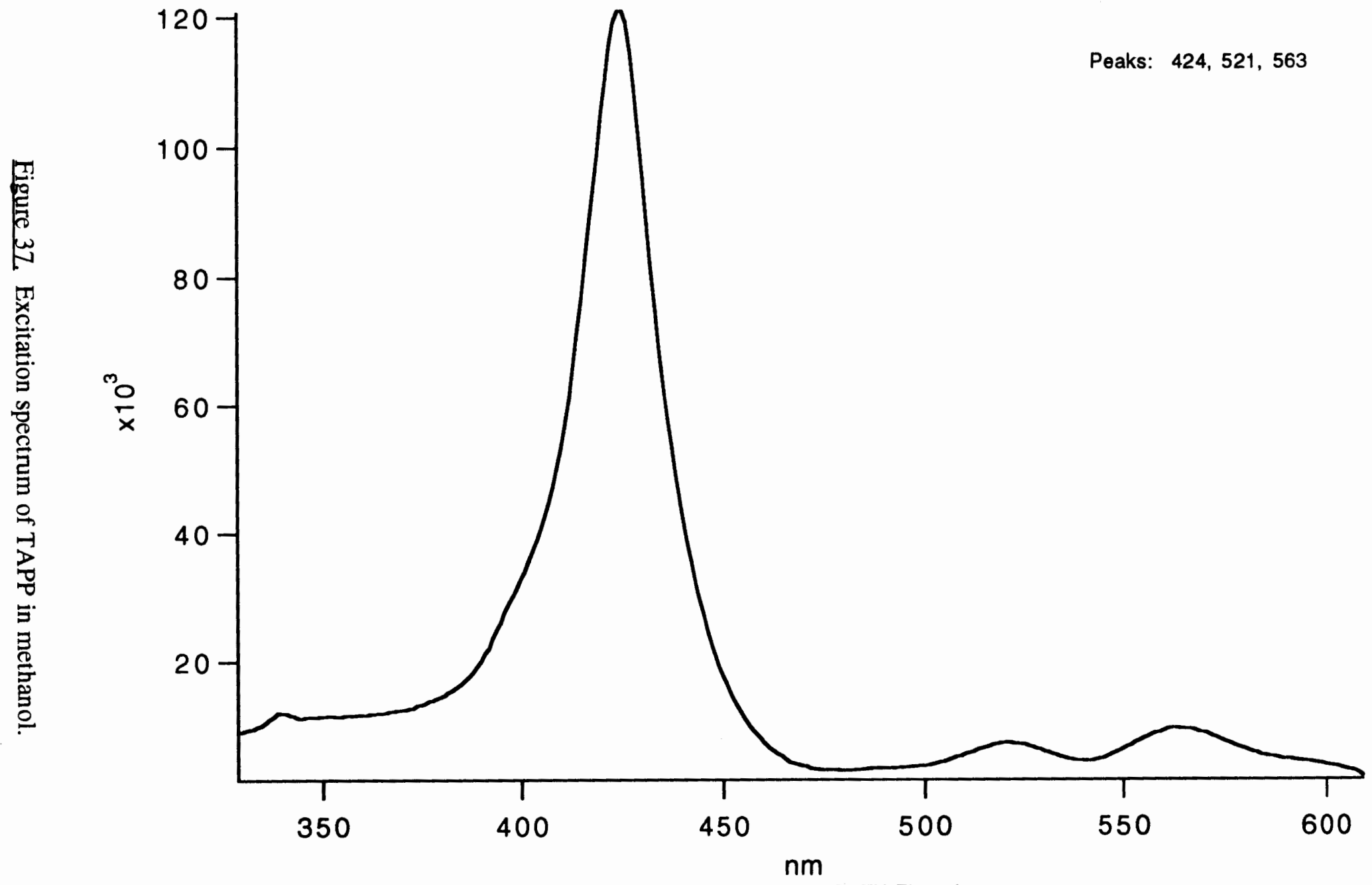

Excitation spectrum of 1e-6 M TAPP in $\mathrm{MeOH}$; detection at $682 \mathrm{~nm}$; SPEX Fluorolog;

range $=330-610 ; 1 \mathrm{~nm}$ incr.; 1 sec. integ. time; PMT voltages $=990 / 580 ;$ acq. mode $=7$ (S1/S2);

exc. slits $=0.5 \mathrm{~mm}$; em. slits $=5.0 \mathrm{~mm} ; 1 \mathrm{~cm}$ quartz cell; right angle detection; corrected for source variation;

Igor expt. "Excitation"; NB p. 94; Date: 2-13-95 


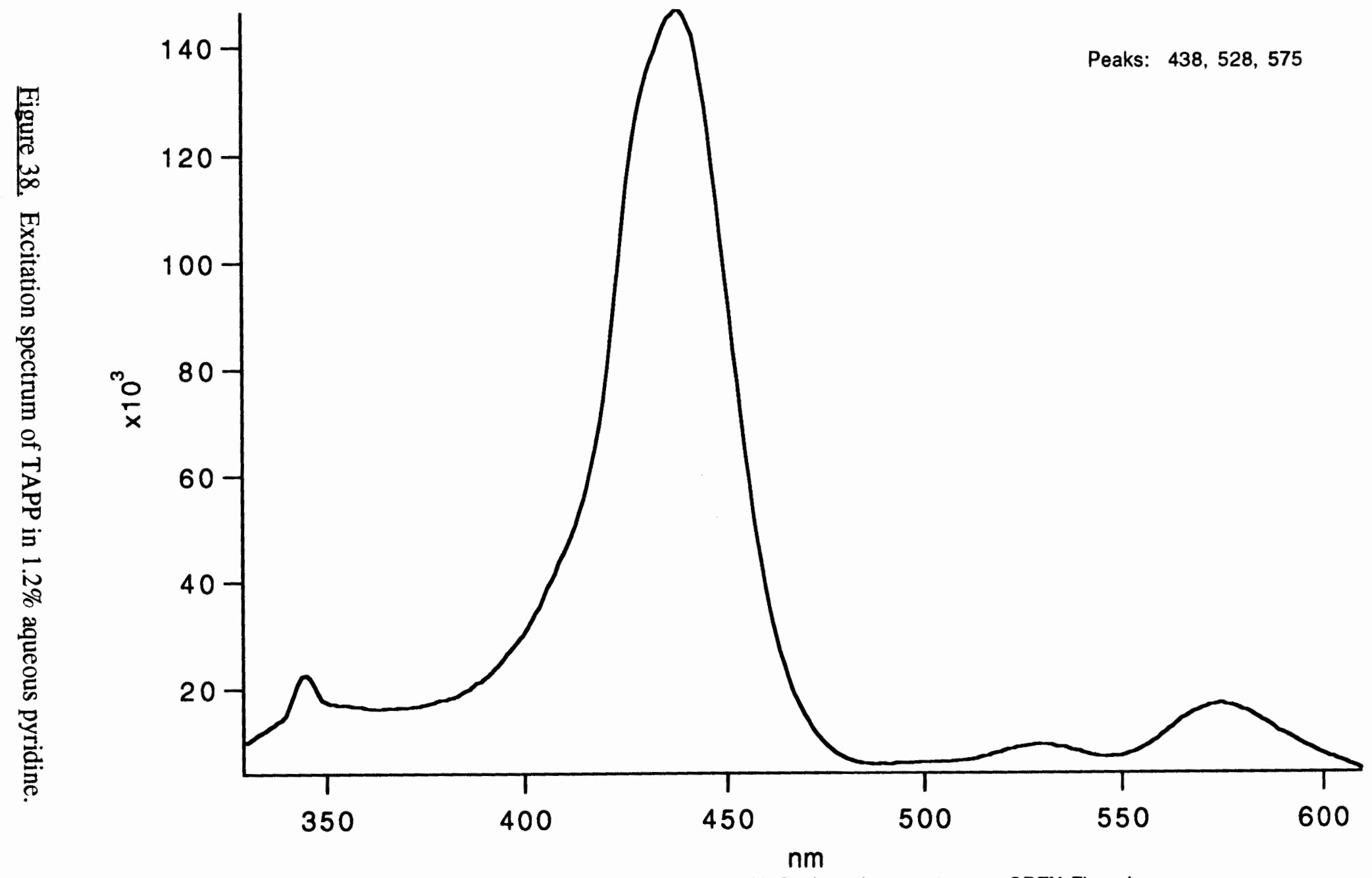

Excitation spectrum of 1.0e-6 M TAPP in pyridine $w / 1.2 \% \mathrm{H} 2 \mathrm{O}$; detection at $691 \mathrm{~nm}$; SPEX Fluorolog; range $=330-610 ; 1 \mathrm{~nm}$ incr.; 1 sec. integ. time; PMT voltages $=990 / 580 ;$ acq. mode $=7$ (S1/S2); exc. slits $=0.5 \mathrm{~mm}$; em. slits $=5.0 \mathrm{~mm} ; 1 \mathrm{~cm}$ quartz cell; right angle detection; corrected for source variation; lgor expt. "Excitation"; NB1 p. 73; Date: 12-20-94 


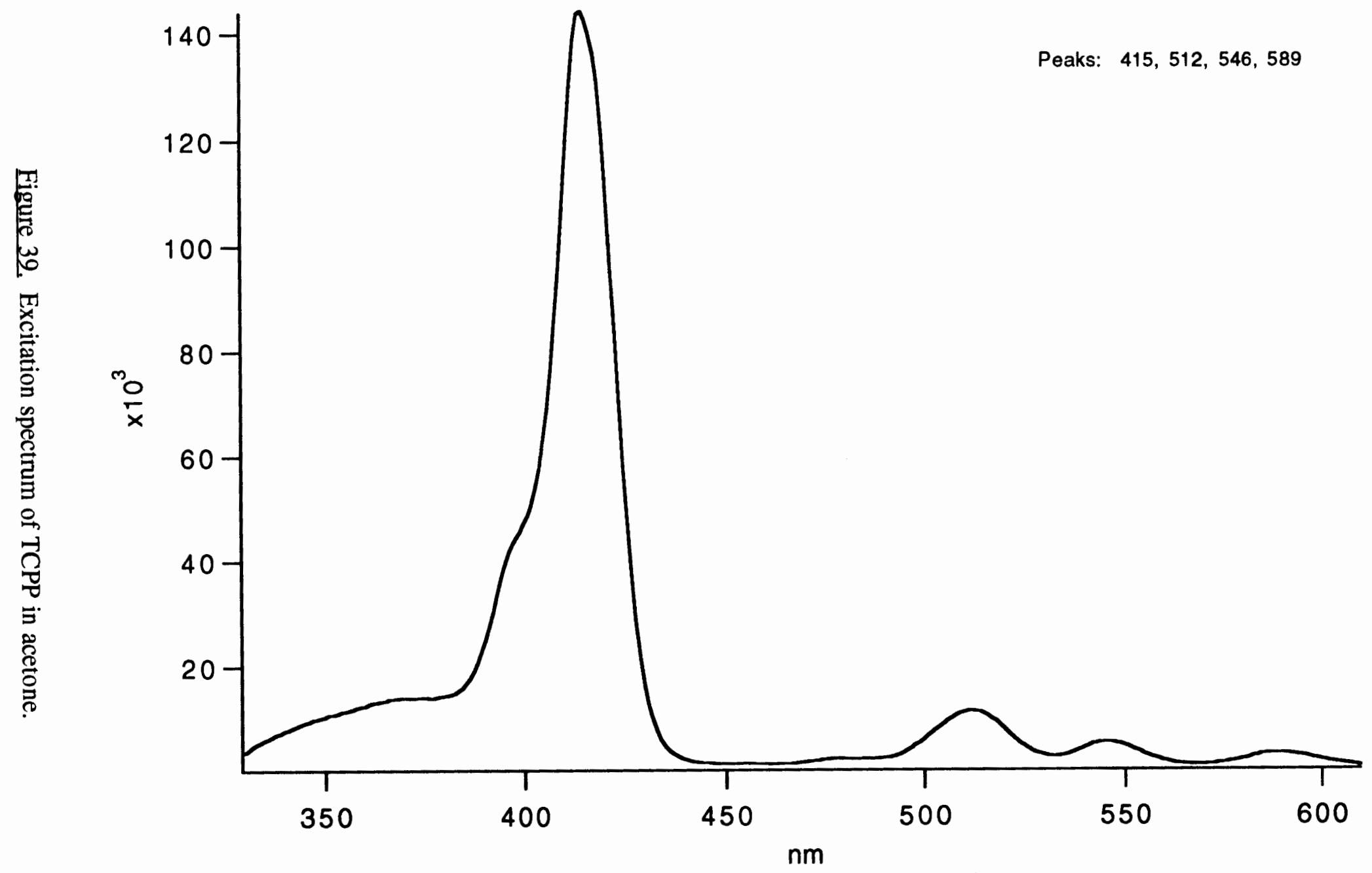

Excitation spectrum of 1e-6 M TCPP in acetone; detection at $650 \mathrm{~nm}$; SPEX Fluorolog;

range = 330-610; $1 \mathrm{~nm}$ incr.; 1 sec. integ. time; PMT voltages $=990 / 580 ;$ acq. mode $=7$ (S1/S2);

exc. slits $=0.5 \mathrm{~mm} ;$ em. slits $=5.0 \mathrm{~mm} ; 1 \mathrm{~cm}$ quartz cell; right angle detection; corrected for source variation;

Igor expt. "Excitation"; NB p. 94; Date: 2-13-95 


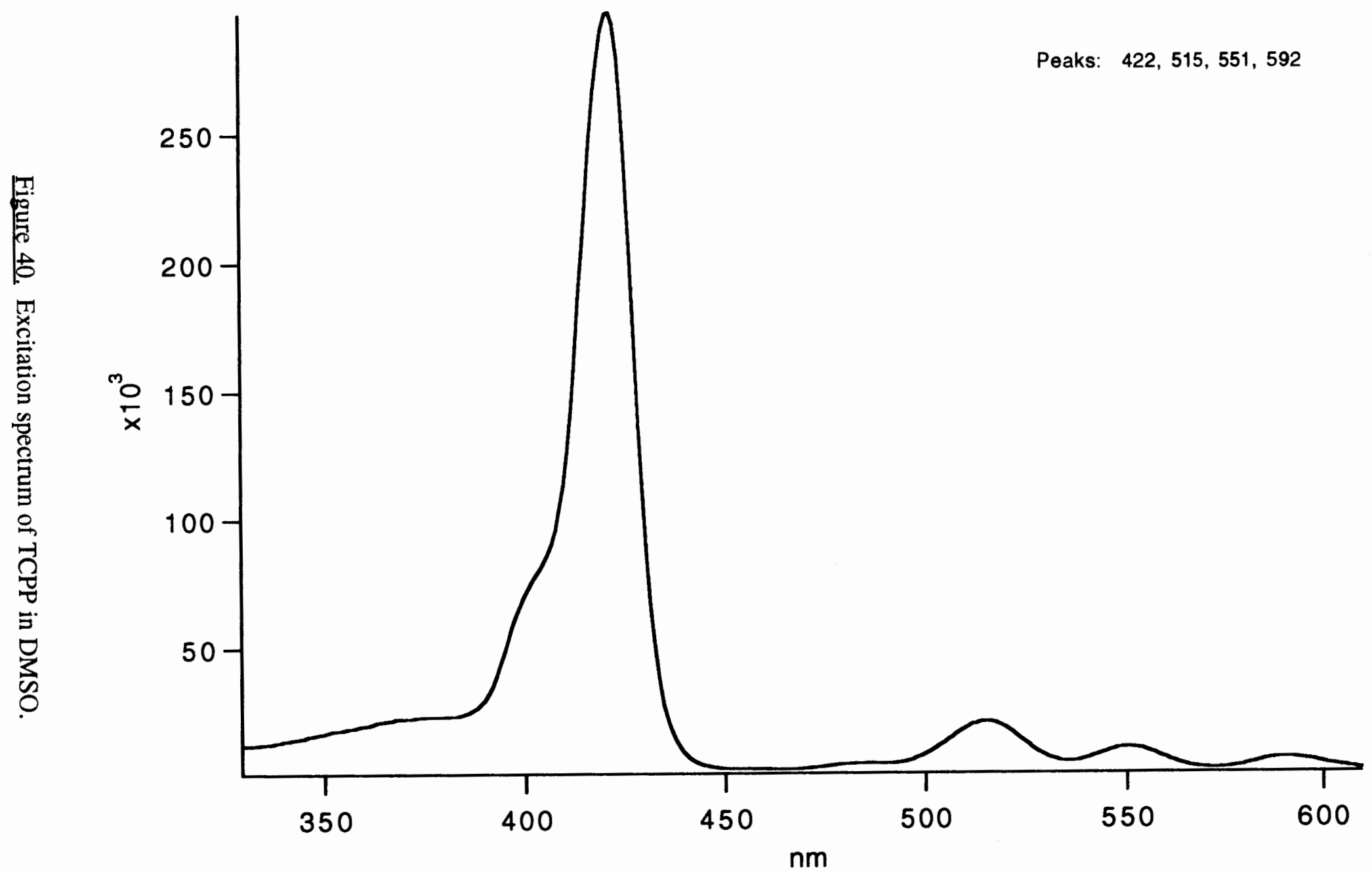

Excitation spectrum of 1.00-6 M TCPP in DMSO; detection at $650 \mathrm{~nm}$; SPEX Fluorolog;

range $=330-610 ; 1 \mathrm{~nm}$ incr.; 1 sec. integ. time; PMT voltages = 990/580; acq. $\bmod \theta=7$ (S1/S2);

exc. slits $=0.5 \mathrm{~mm}$; em. slits $=5.0 \mathrm{~mm} ; 1 \mathrm{~cm}$ quartz cell; right angle detection; corrected for source variation;

Igor expt. "Excitation"; NB2 p. 28; Date: 5-22-95 


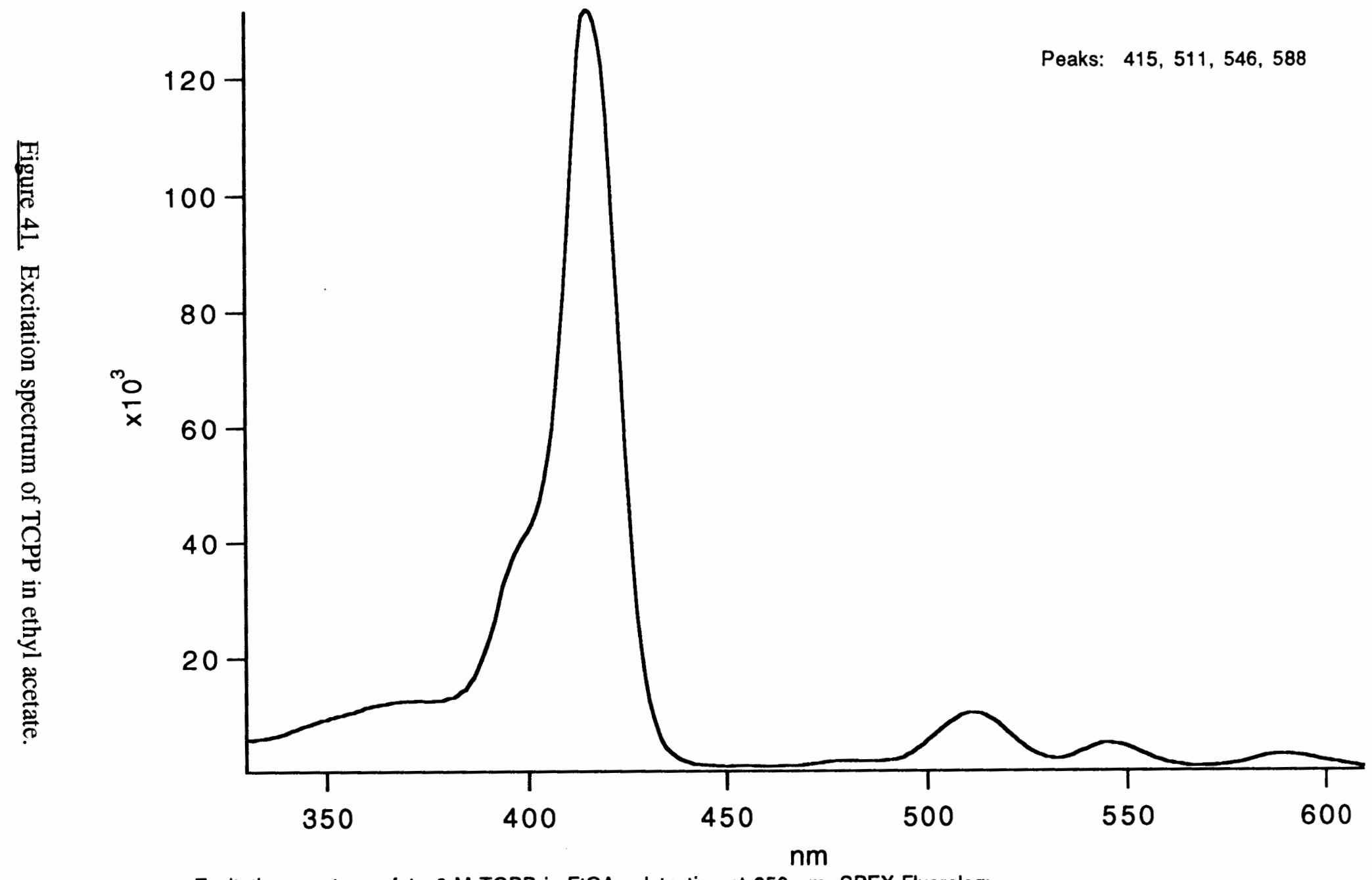

Excitation spectrum of 10-6 M TCPP in EtOAc; detection at $650 \mathrm{~nm}$; SPEX Fluorolog;

range = 330-610; $1 \mathrm{~nm}$ incr.; 1 sec. integ. time; PMT voltages = 990/580; acq. mode = 7 (S1/S2);

exc. slits $=0.5 \mathrm{~mm} ;$ em. slits $=5.0 \mathrm{~mm} ; 1 \mathrm{~cm}$ quartz cell; right angle detection; corrected for source variation;

Igor expt. "Excitation"; NB p. 94; Date: 2-13-95 


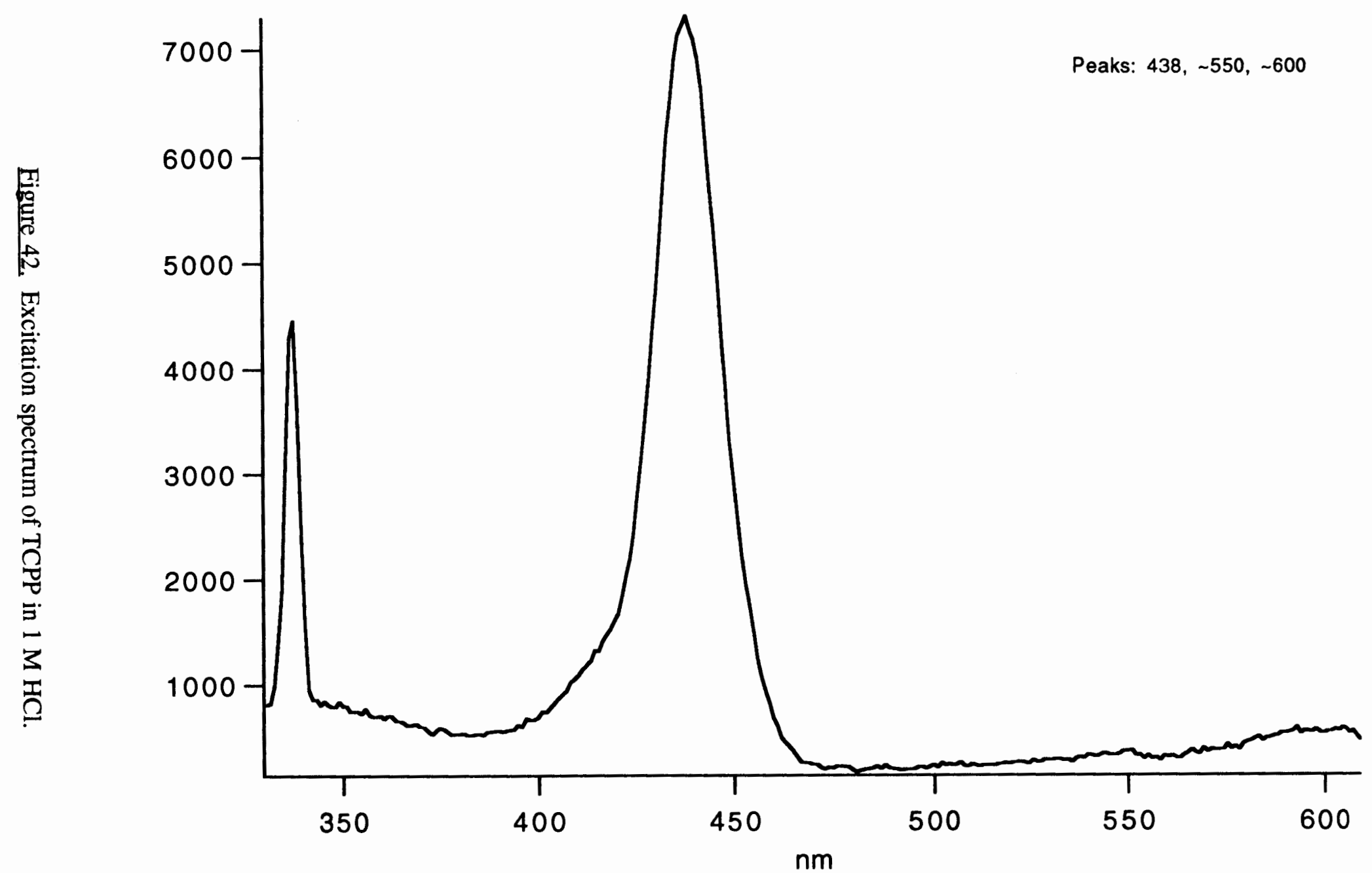

Excitation spectrum of 2e-6 $\mathrm{M}$ TCPP in $1 \mathrm{M} \mathrm{HCl}$; detection at $675 \mathrm{~nm}$; SPEX Fluorolog;

range = 330-610; $1 \mathrm{~nm}$ incr.; 1 sec. integ. time; PMT voltages = 990/580; acq. mode $=7$ (S1/S2);

exc. slits $=0.5 \mathrm{~mm}$; em. slits $=5.0 \mathrm{~mm} ; 1 \mathrm{~cm}$ quartz cell; right angle detection; corrected for source variation;

Igor expt. "Excitation"; NB2 p. 12; Date: 4-13-95 


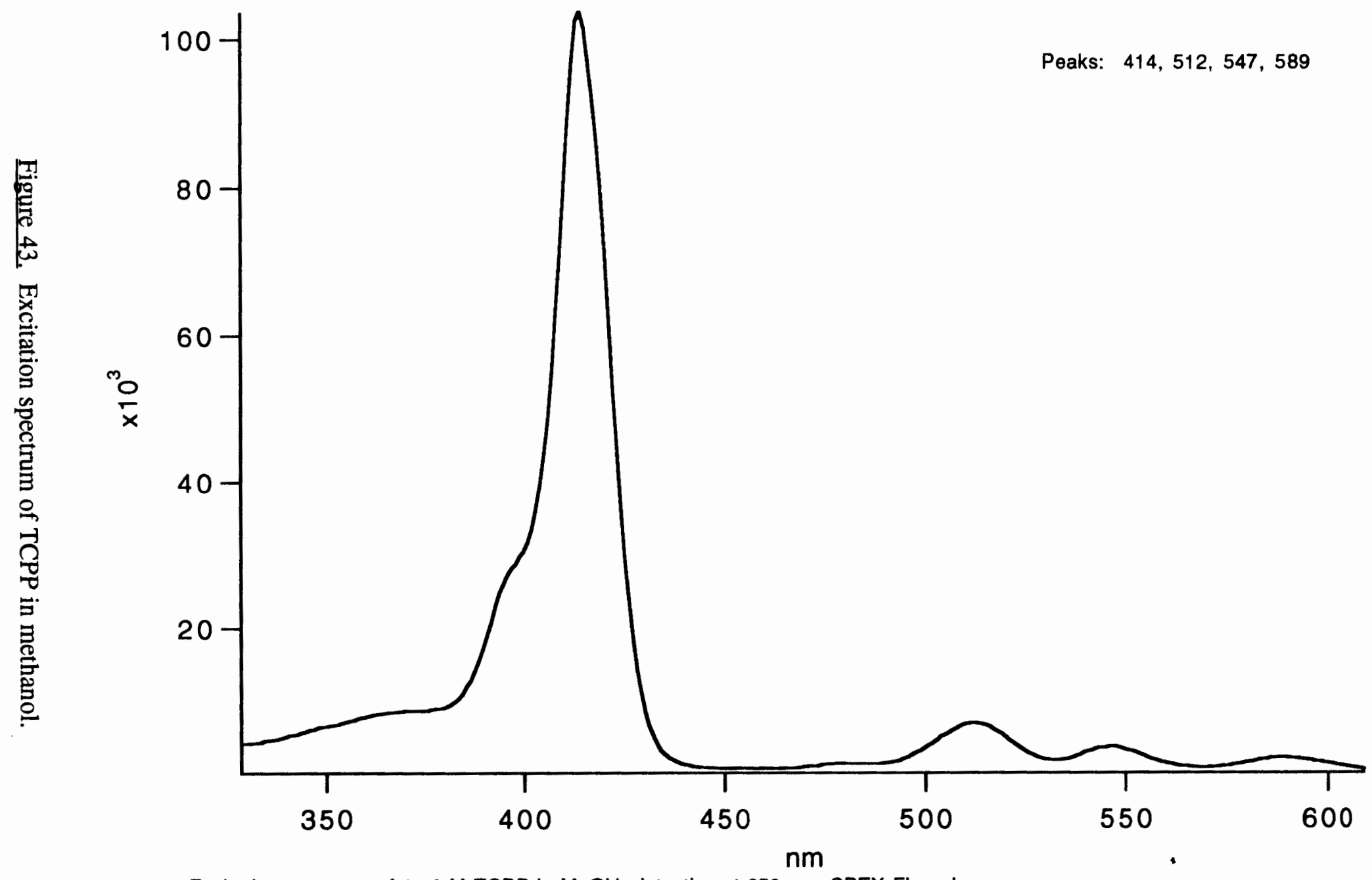

Excitation spectrum of 1e-6 M TCPP in $\mathrm{MeOH}$; detection at $650 \mathrm{~nm}$; SPEX Fluorolog;

range = 330-610; $1 \mathrm{~nm}$ incr.; 1 sec. integ. time; PMT voltages = 990/580; acq. mode = 7 (S1/S2);

exc. slits $=0.5 \mathrm{~mm}$; em. slits $=5.0 \mathrm{~mm} ; 1 \mathrm{~cm}$ quartz cell; right angle detection; corrected for source variation;

Igor expt. "Excitation"; NB p. 94; Date: 2-13-95 


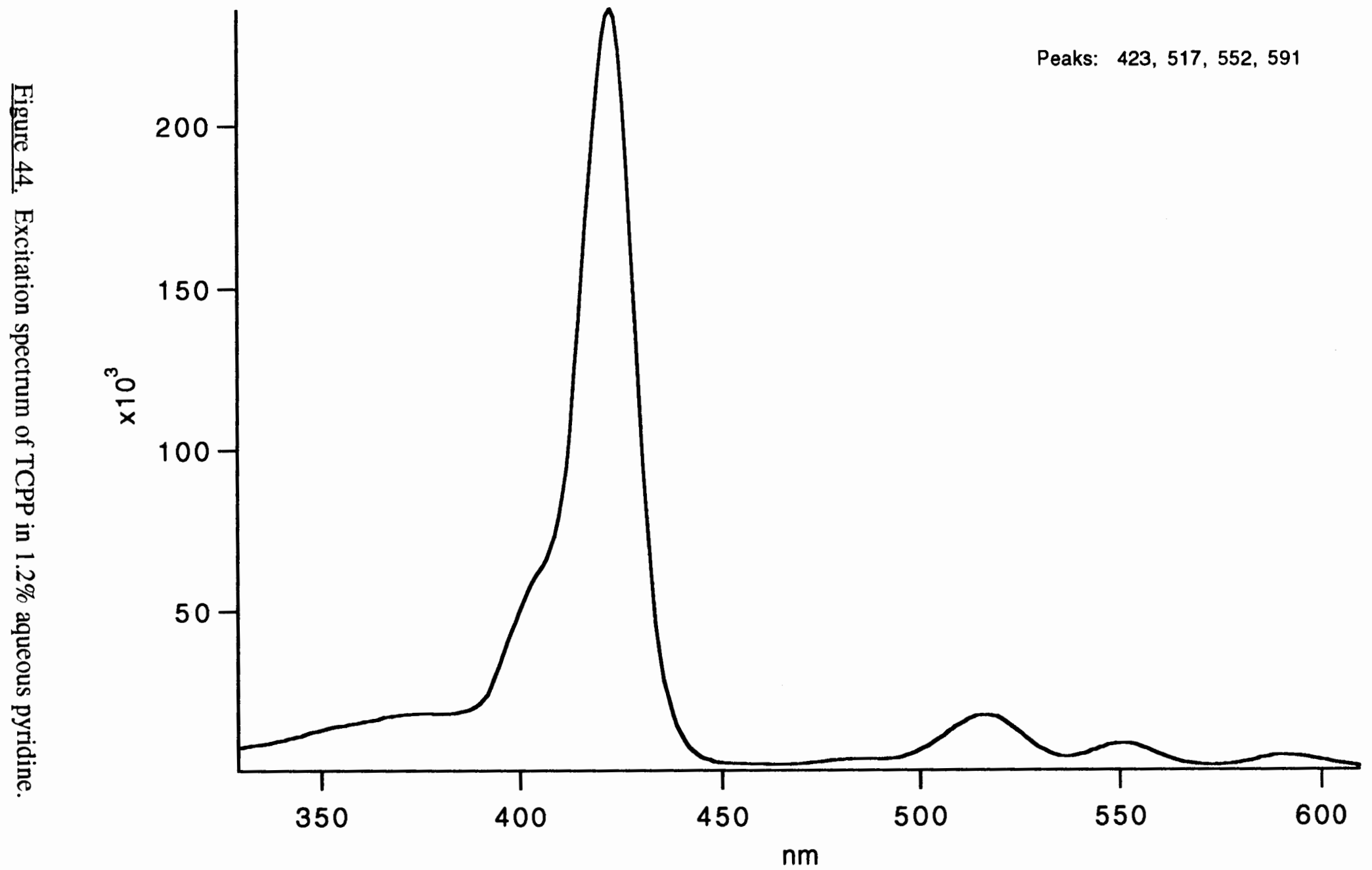

Excitation spectrum of 1.0e-6 M TCPP in pyridine $w / 1.2 \% \mathrm{H} 2 \mathrm{O}$; detection at $650 \mathrm{~nm}$; SPEX Fluorolog; range = 330-610; $1 \mathrm{~nm}$ incr.; 1 sec. integ. time; PMT voltages = 990/580; acq. $\operatorname{mode}=7$ (S1/S2);

exc. slits $=0.5 \mathrm{~mm}$; em. slits $=5.0 \mathrm{~mm} ; 1 \mathrm{~cm}$ quartz cell; right angle detection; corrected for source variation;

Igor expt. "Excitation"; NB1 p. 72; Date: 12-18-94 


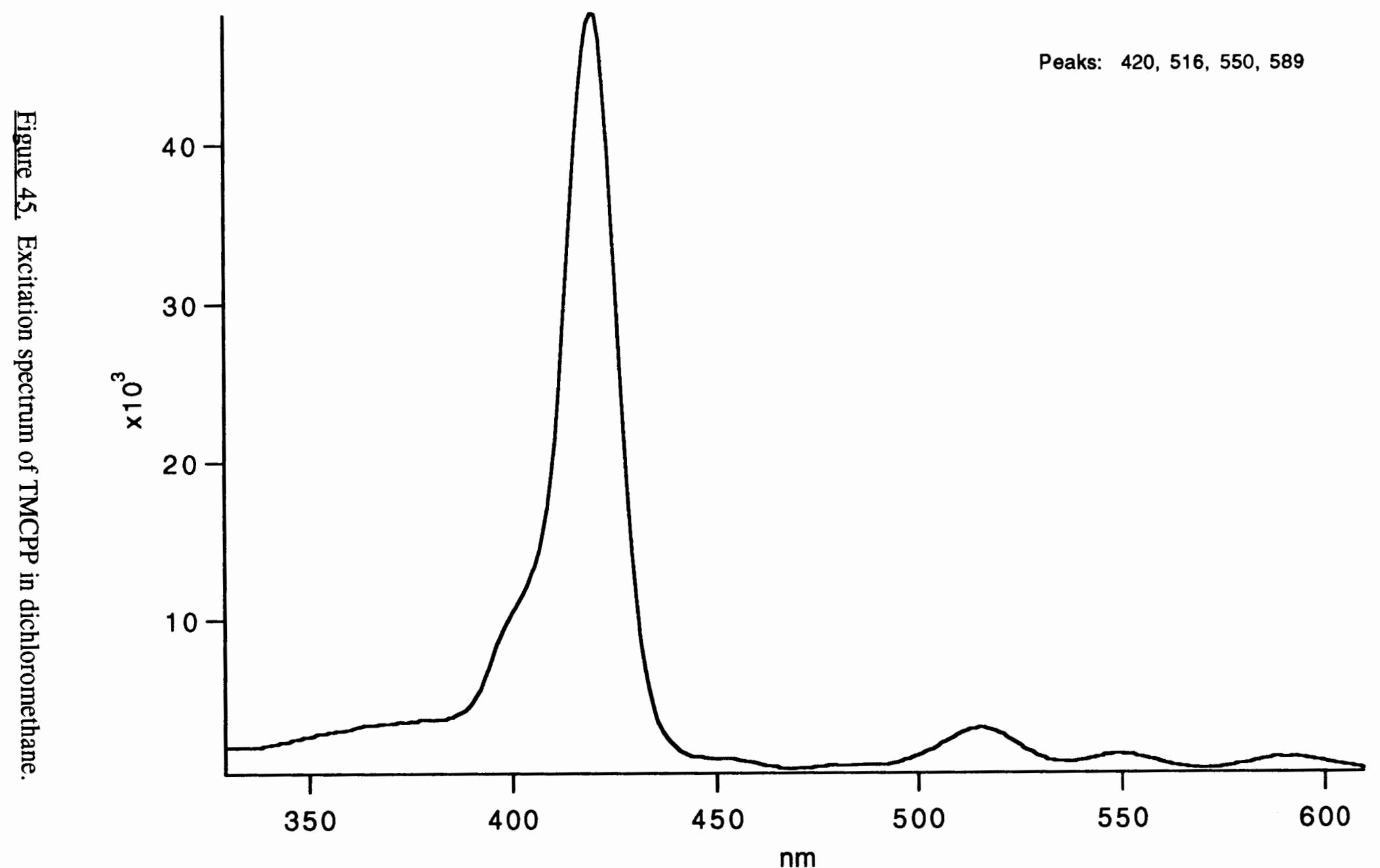

Excitation spectrum of TMCPP in DCM, unk. conc.; detection at $650 \mathrm{~nm}$; SPEX Fluorolog;

range $=330-610 ; 1 \mathrm{~nm}$ incr.; 1 sec. integ. time; PMT voltages $=990 / 580 ;$ acq. mode $=7$ (S1/S2);

exc. slits $=0.5 \mathrm{~mm} ;$ em. slits $=5.0 \mathrm{~mm} ; 1 \mathrm{~cm}$ quartz cell; right angle detection; corrected for source variation;

Igor expt. "Excitation"; NB1 p. 95; Date: 3-19-95 


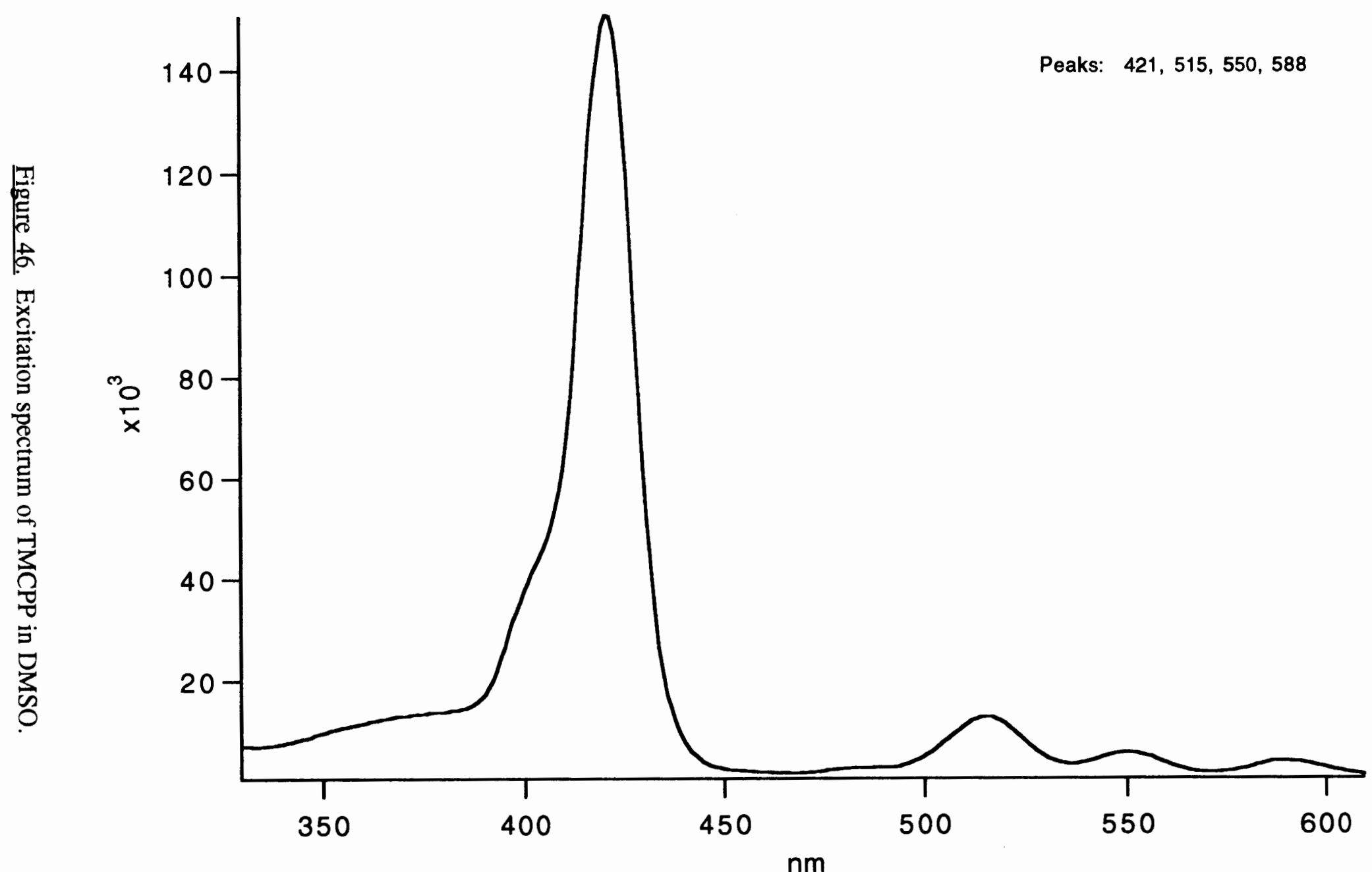

Excitation spectrum of TMCPP in DMSO, unknown conc.; detection at $650 \mathrm{~nm}$; SPEX Fluorolog;

range = 330-610; $1 \mathrm{~nm}$ incr.; $1 \mathrm{sec}$. integ. time; PMT voltages = 990/580; acq. mode = 7 (S1/S2);

exc. slits $=0.5 \mathrm{~mm} ;$ em. slits $=5.0 \mathrm{~mm} ; 1 \mathrm{~cm}$ quartz cell; right angle detection; corrected for source variation;

lgor expt. "Excitation"; NB1 p. 86; Date: 1-25-95 


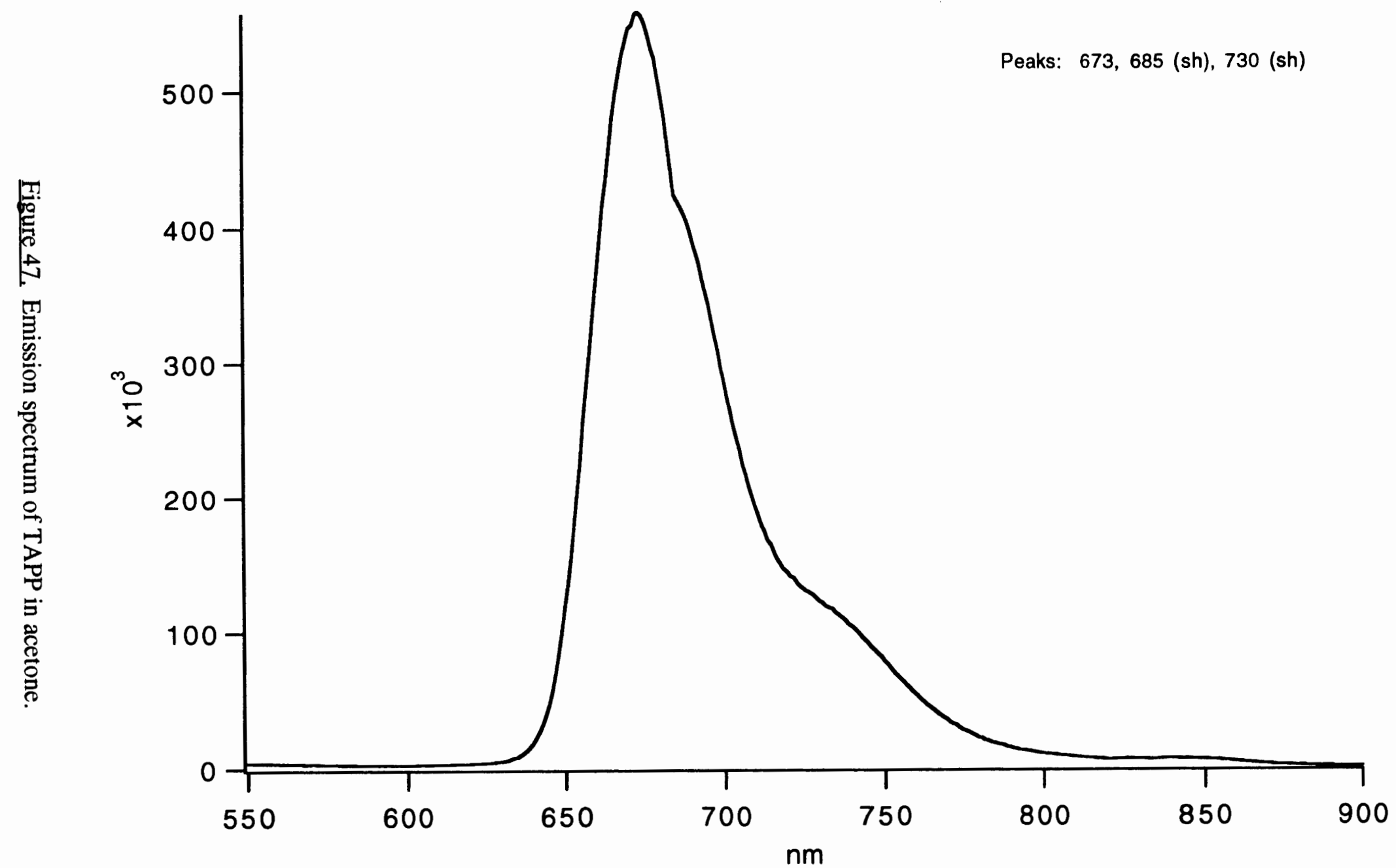

Emission spectrum of 1e-6 M TAPP in acetone; excitation at $427 \mathrm{~nm}$; SPEX Fluorolog; range $=550-900 ; 1 \mathrm{~nm}$ incr.; 1 sec. integ. time; PMT voltages $=990 / 0 ;$ acq. mode $=0$ (S1);

exc. slits $=5.0 \mathrm{~mm}$; em. slits $=0.5 \mathrm{~mm} ; 1 \mathrm{~cm}$ quartz cell; right angle detection; not corrected for blank; Igor expt. "Emission"; NB p. 94; Date: 2-13-95 


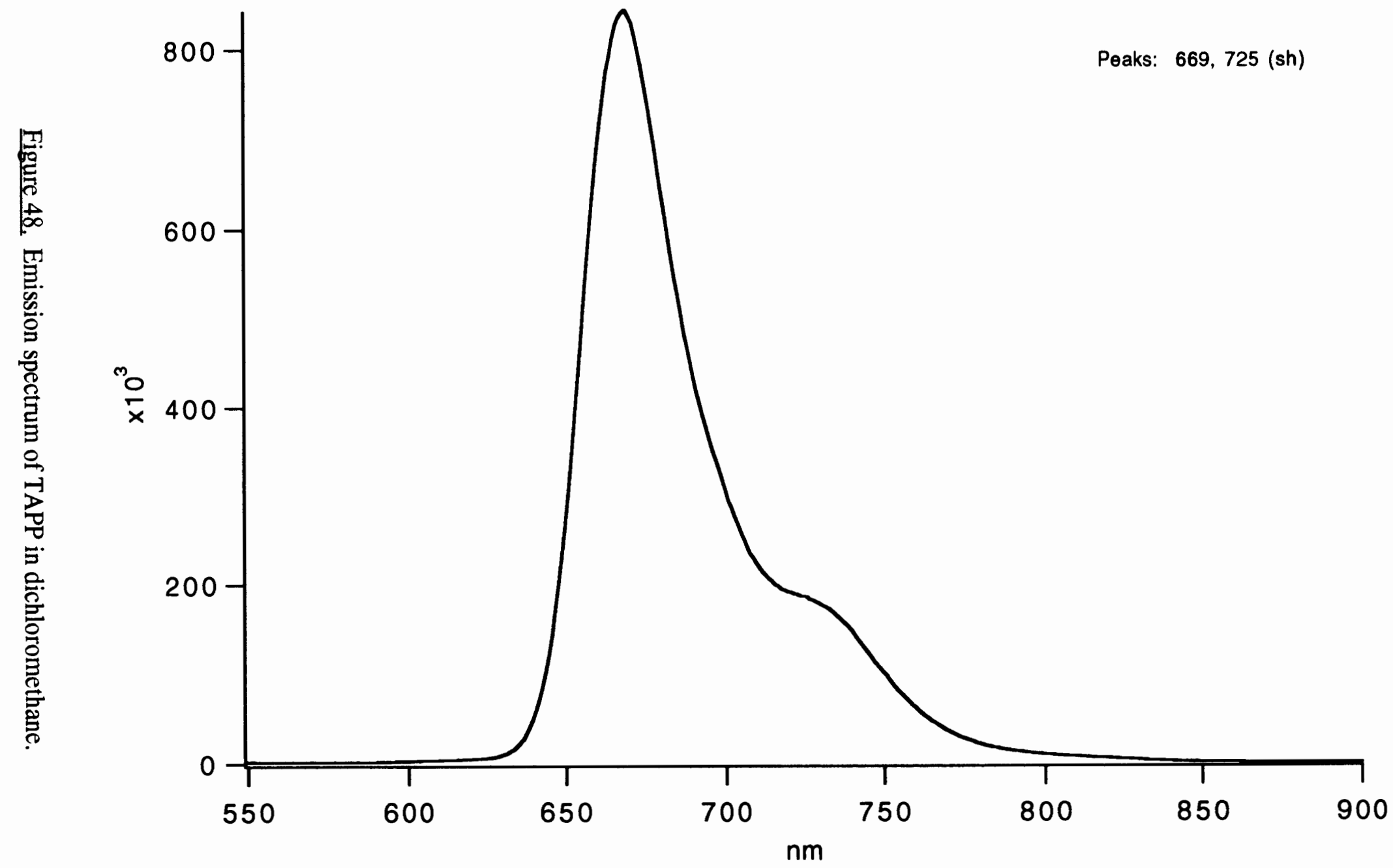

Emission spectrum of TAPP in $\mathrm{CH}_{2} \mathrm{Cl} 2$; excitation at $427 \mathrm{~nm}$; SPEX Fluorolog;

range = 550-900; $1 \mathrm{~nm}$ incr.; 1 sec. integ. time; PMT voltages = 950/120; acq. $\operatorname{mode}=6$ (KS1-S2);

exc. slits $=5.0 \mathrm{~mm}$; em. slits $=0.5 \mathrm{~mm} ; 1 \mathrm{~cm}$ quartz cell; right angle detection; corrected for blank;

Igor expt. "Emission"; NB p. 97; Date: 2-24-95 


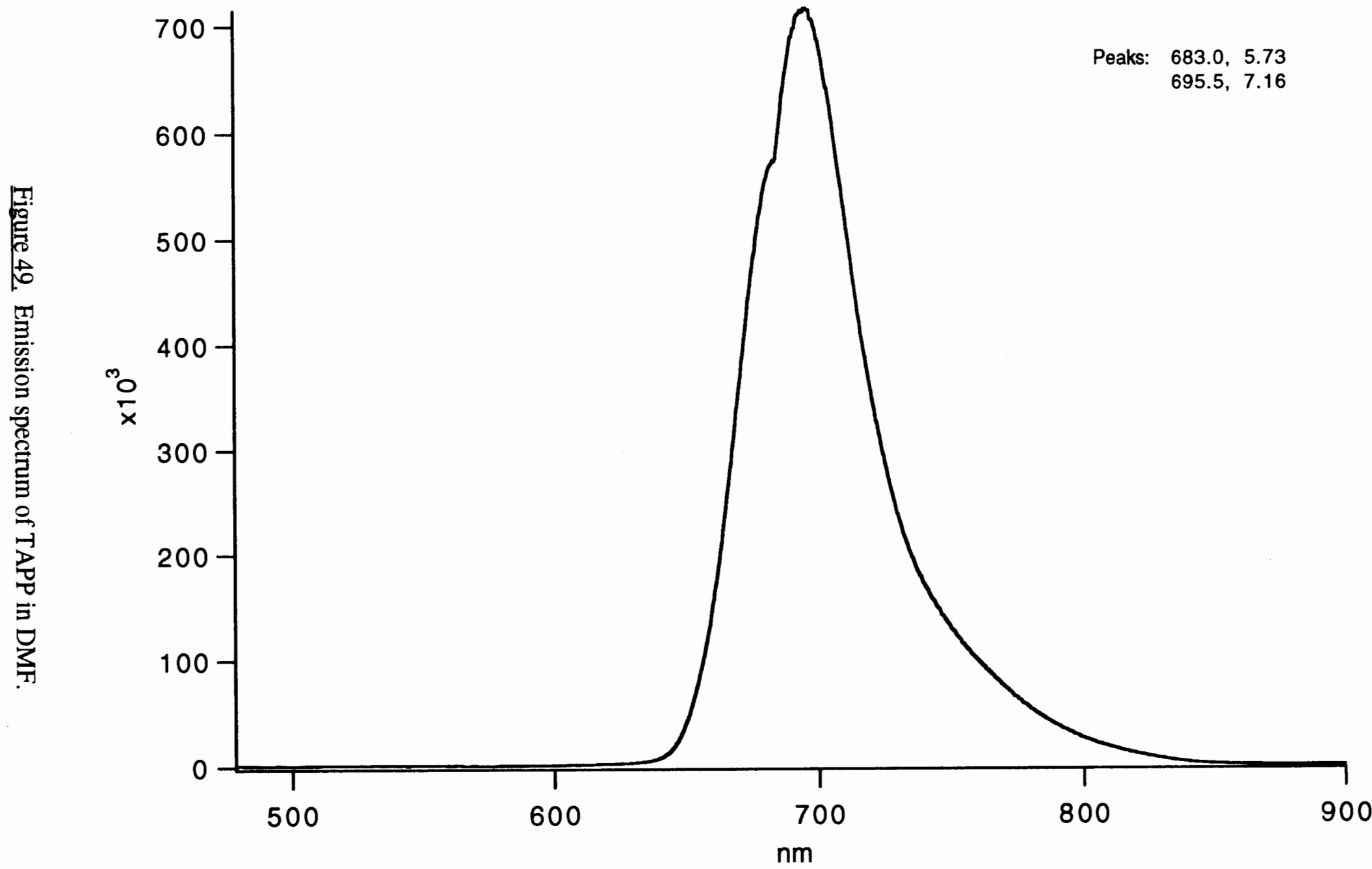

Emission spectrum of 2.5E-5 M TAPP in DMF; exc. at $450 \mathrm{~nm}$; SPEX Fluorolog; range $=480-900 \mathrm{~nm}$;

PMT voltages $=950 / 180 \mathrm{~V} ; 1 \mathrm{~cm}$ quartz cell; corrected for blank; aeq. mode $=6$ (KS1-S2); $0.5 \mathrm{~nm}$ incr.;

1 sec. integ. time; exc. slits $=5.0 \mathrm{~mm}$; em. slits $=0.5 \mathrm{~mm}$; right angle detection; Date: 7-8-94;

Igor expt. "Emission"

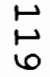




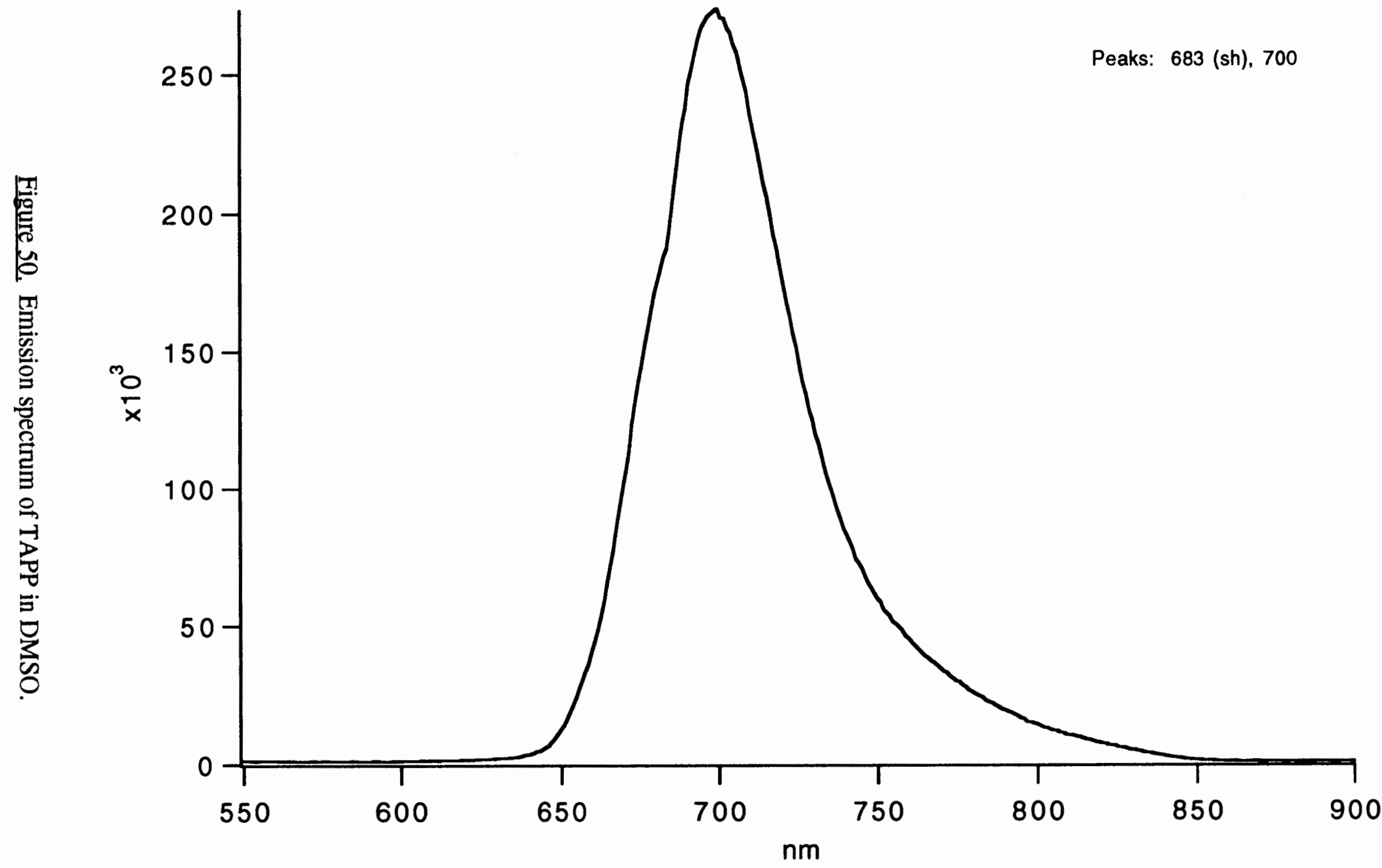

Emission spectrum of 1.00e-6 M TAPP in DMSO; excitation at $441 \mathrm{~nm}$; SPEX Fluorolog; range = 550-900; $1 \mathrm{~nm}$ incr.; 1 sec. integ. time; PMT voltages = 990/120; acq. mode = 6 (KS1-S2); exc. slits $=5.0 \mathrm{~mm}$; em. slits $=0.5 \mathrm{~mm} ; 1 \mathrm{~cm}$ quartz cell; right angle detection; corrected for blank; Igor expt. "Emission"; NB2 p. 28; Date: 5-22-95 


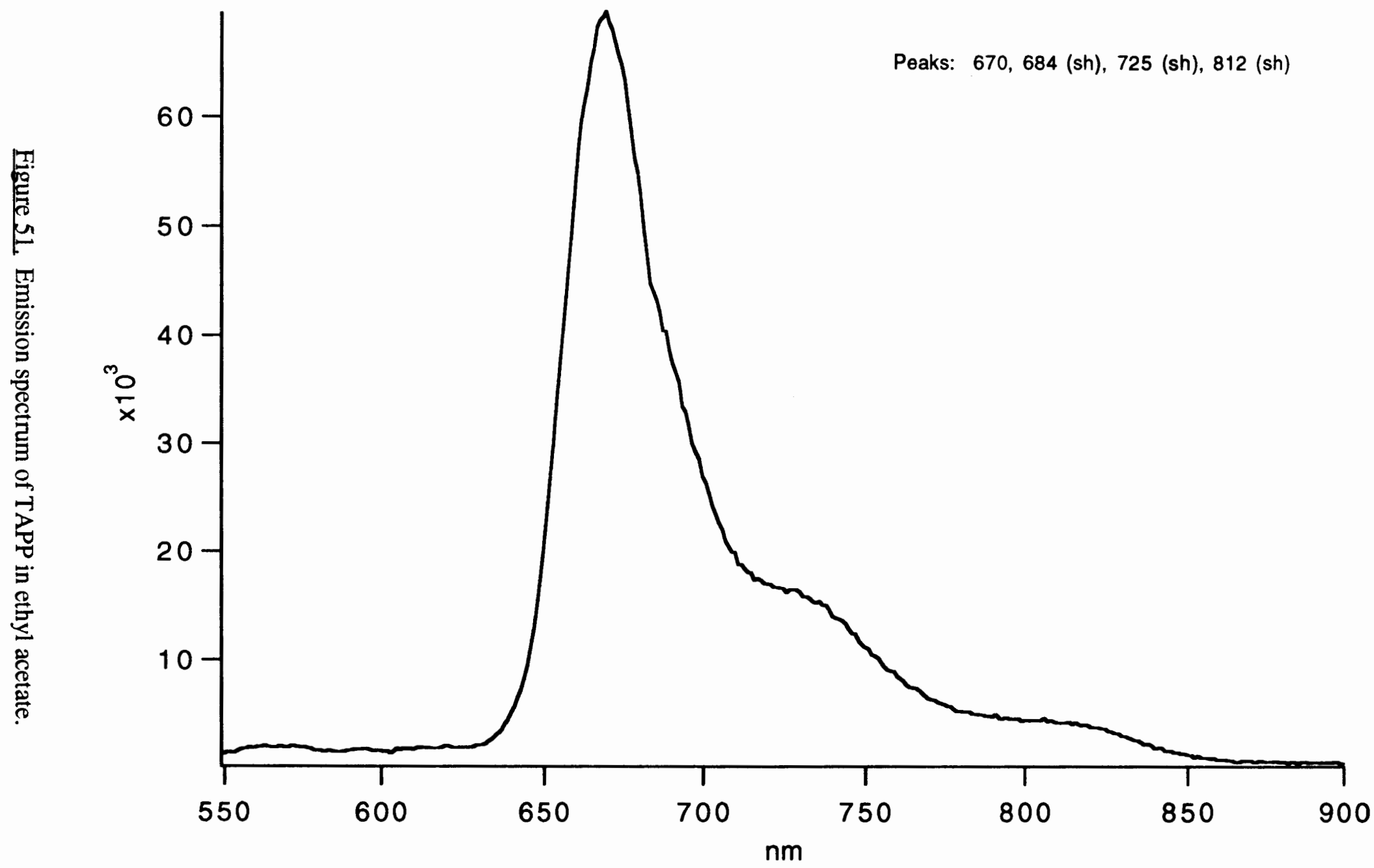

Emission spectrum of 1e-6 M TAPP in EtOAc; excitation at $427 \mathrm{~nm}$; SPEX Fluorolog;

range $=550-900 ; 1 \mathrm{~nm}$ incr.; 1 sec. integ. time; PMT voltages $=990 / 0 ;$ acq. mode $=0$ (S1);

exc. slits $=5.0 \mathrm{~mm}$; em. slits $=0.5 \mathrm{~mm} ; 1 \mathrm{~cm}$ quartz cell; right angle detection; not corrected for blank:

Igor expt. "Emission"; NB p. 94; Date: 2-13-95 


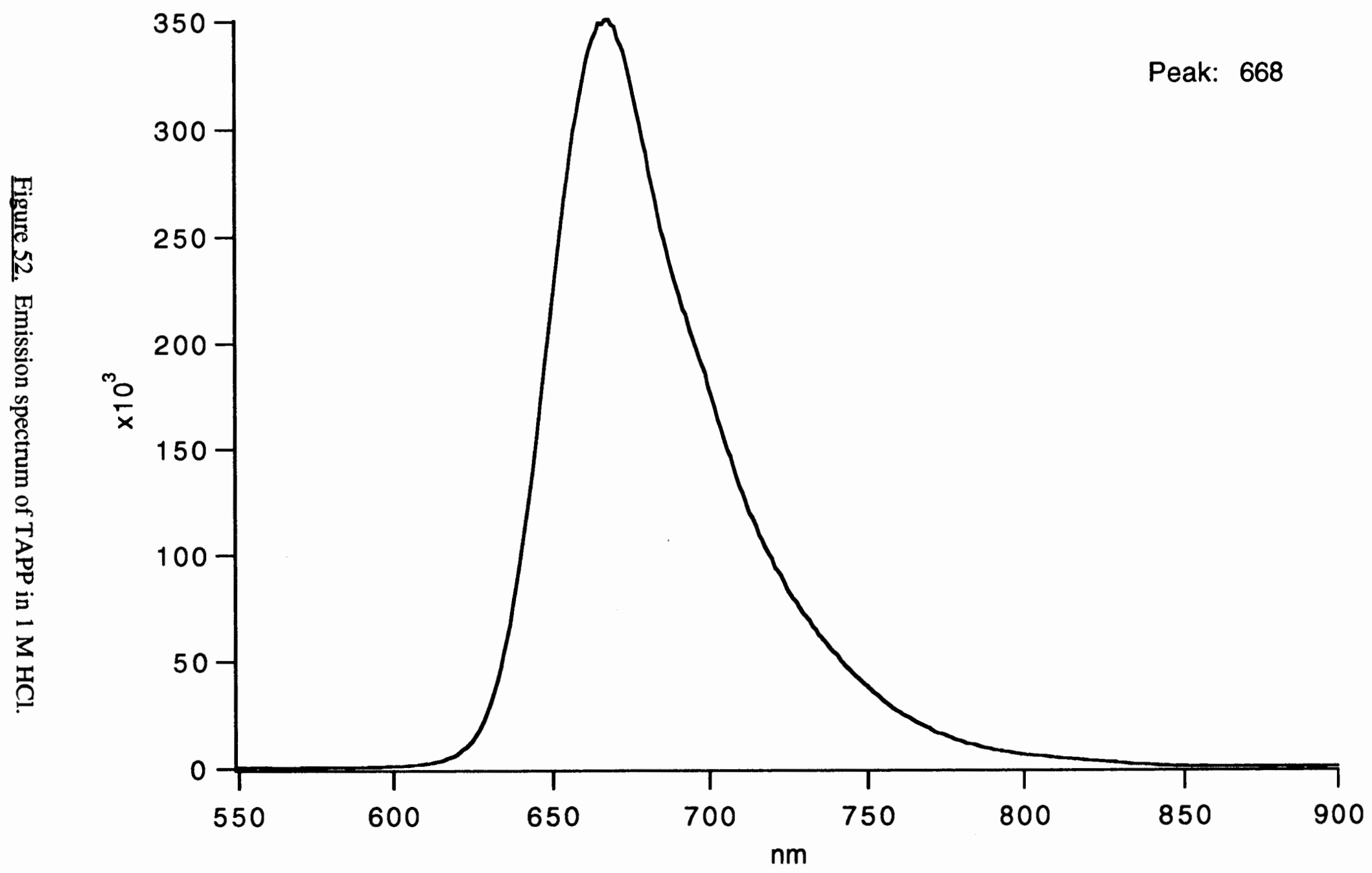

Emission spectrum of 2e-6 M TAPP in $1 \mathrm{M} \mathrm{HCl}$; excitation at $429 \mathrm{~nm}$; SPEX Fluorolog;

range = 550-900; $1 \mathrm{~nm}$ incr.; 1 sec. integ. time; PMT voltages $=990 / 0 ;$ acq. mode $=0$ (S1);

exc. slits $=5.0 \mathrm{~mm} ;$ em. slits $=0.5 \mathrm{~mm} ; 1 \mathrm{~cm}$ quartz cell; right angle detection; not corrected for blank;

Igor expt. "Emission"; NB2 p. 12; Date: 4-13-95 


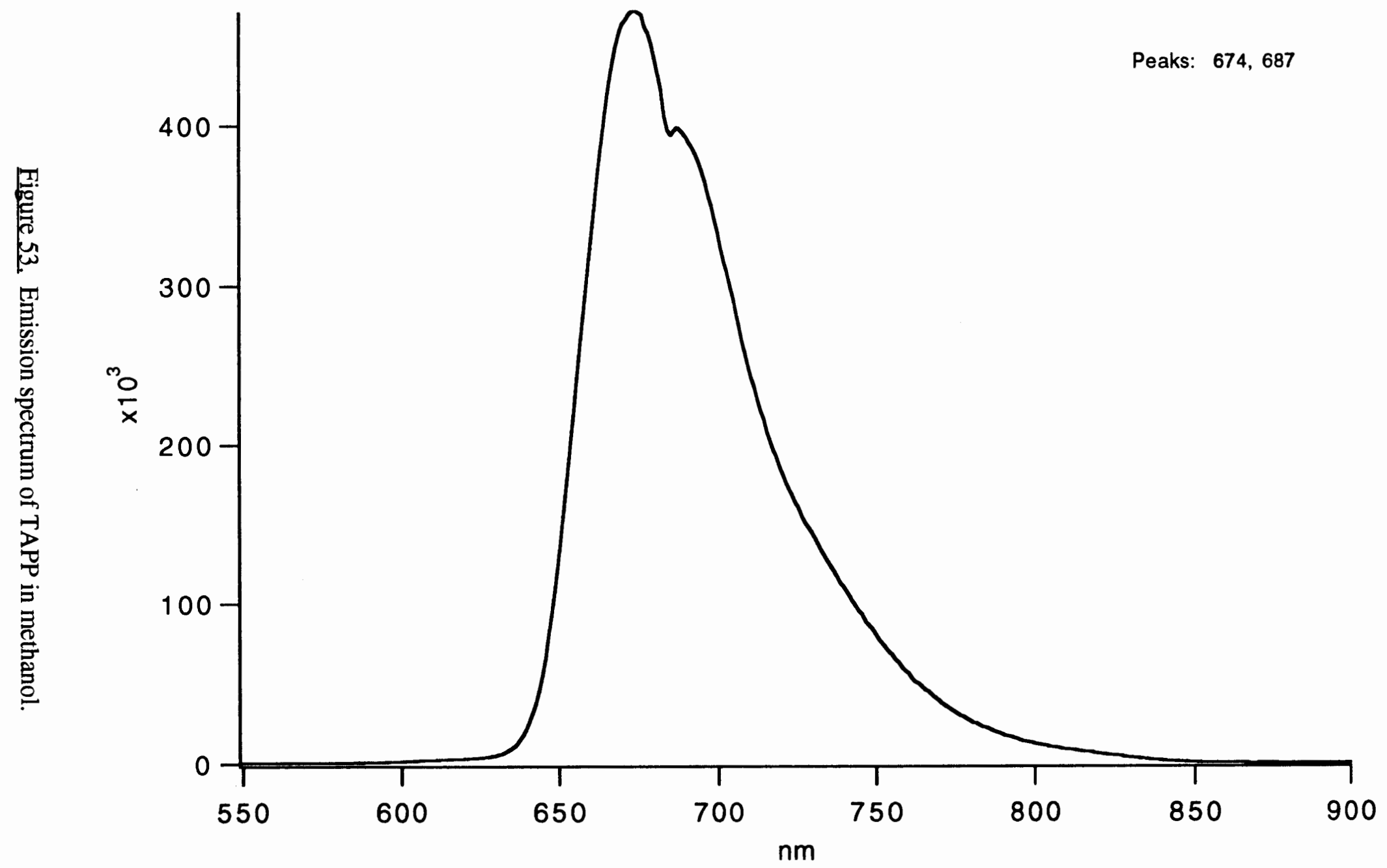

Emission spectrum of 1e-6 M TAPP in $\mathrm{MeOH}$; excitation at $427 \mathrm{~nm}$; SPEX Fluorolog;

range = 550-900; $1 \mathrm{~nm}$ incr.; 1 sec. integ. time; PMT voltages = 990/0; acq. mode = 7 (S1);

exc. slits $=5.0 \mathrm{~mm}$; em. slits $=0.5 \mathrm{~mm} ; 1 \mathrm{~cm}$ quartz cell; right angle detection; not corrected for blank;

Igor expt. "Emission"; NB p. 94; Date: 2-13-95 


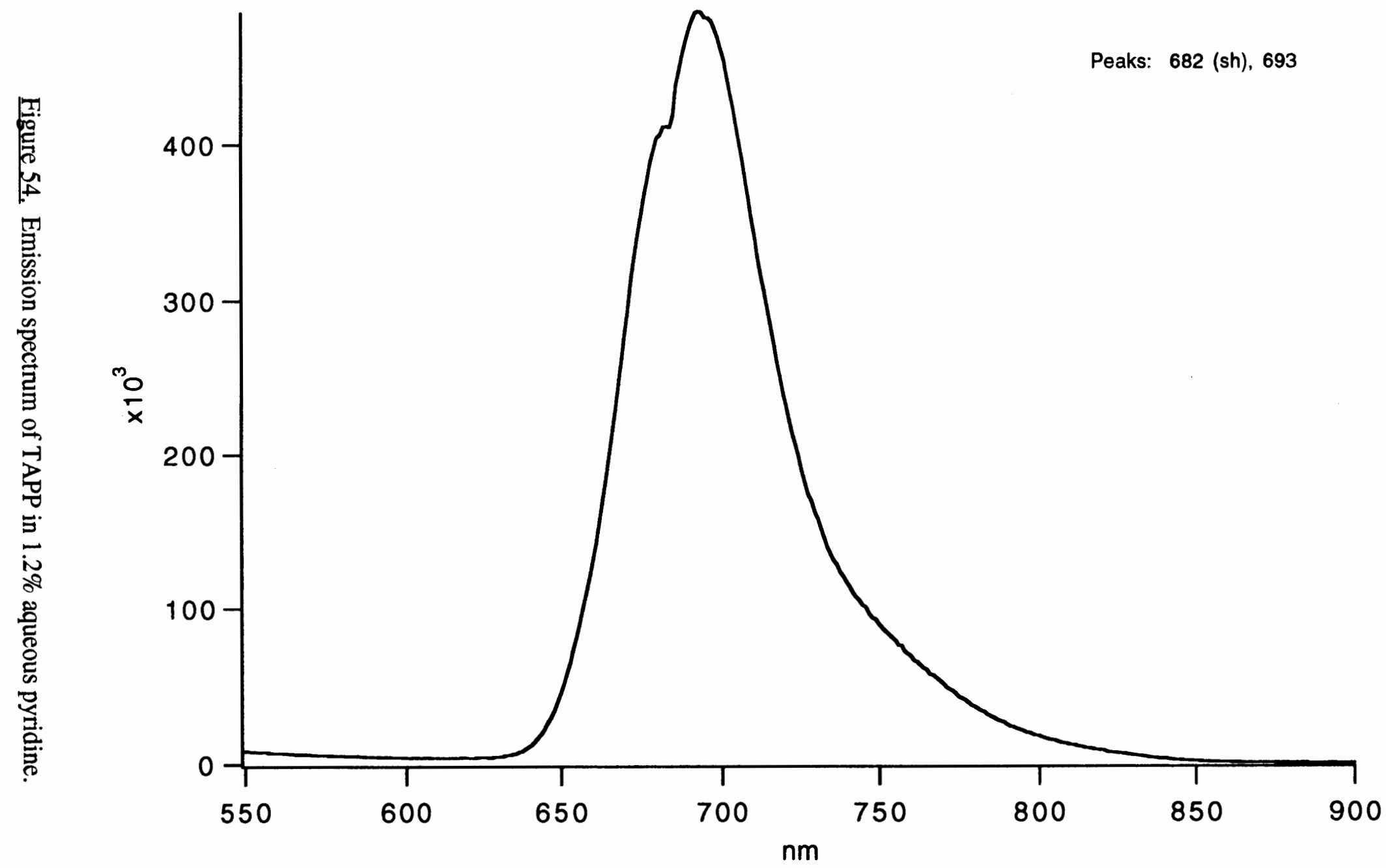

Emission spectrum of 1.00-6 M TAPP in pyridine w/ 1.2\% H2O; excitation at $430 \mathrm{~nm}$; SPEX Fluorolog; range = 550-900; $1 \mathrm{~nm}$ incr.; 1 sec. integ. time; PMT voltages = 990/120; acq. mode = 6 (KS1-S2); exc. slits $=5.0 \mathrm{~mm}$; em. slits $=0.5 \mathrm{~mm} ; 1 \mathrm{~cm}$ quartz cell; right angle detection; corrected for blank; Igor expt. "Emission"; NB1 p. 72; Date: 12-18-94 


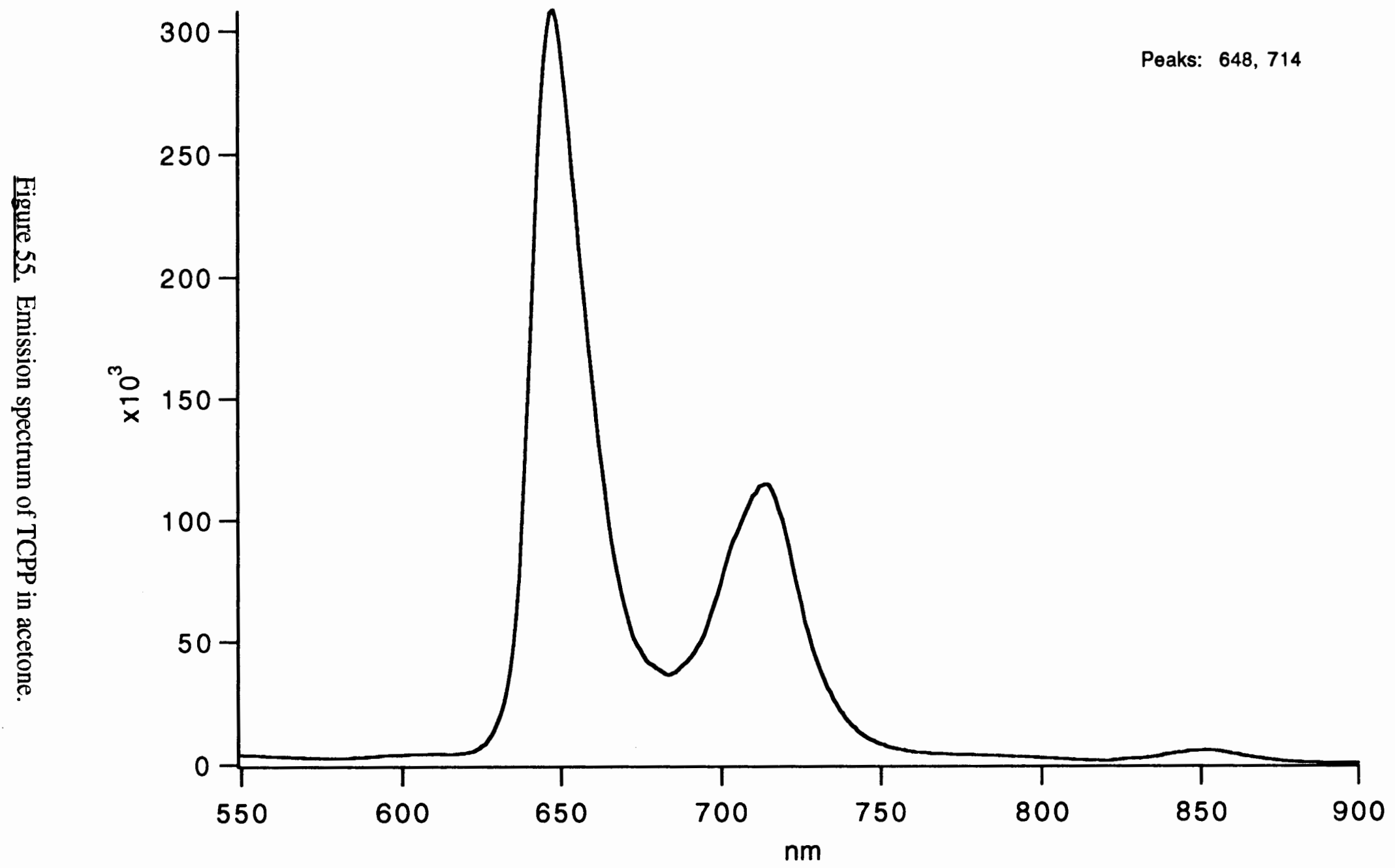

Emission spectrum of 1e-6 M TCPP in acetone; excitation at $427 \mathrm{~nm}$; SPEX Fluorolog;

range = 550-900; $1 \mathrm{~nm}$ incr.; 1 sec. integ. time; PMT voltages = 990/0; acq. mode = $7(S 1)$;

exc. slits $=5.0 \mathrm{~mm}$; $\theta \mathrm{m}$. slits $=0.5 \mathrm{~mm} ; 1 \mathrm{~cm}$ quartz cell; right angle detection; not corrected for blank;

Igor expt. "Emission"; NB p. 94; Date: 2-13-95 


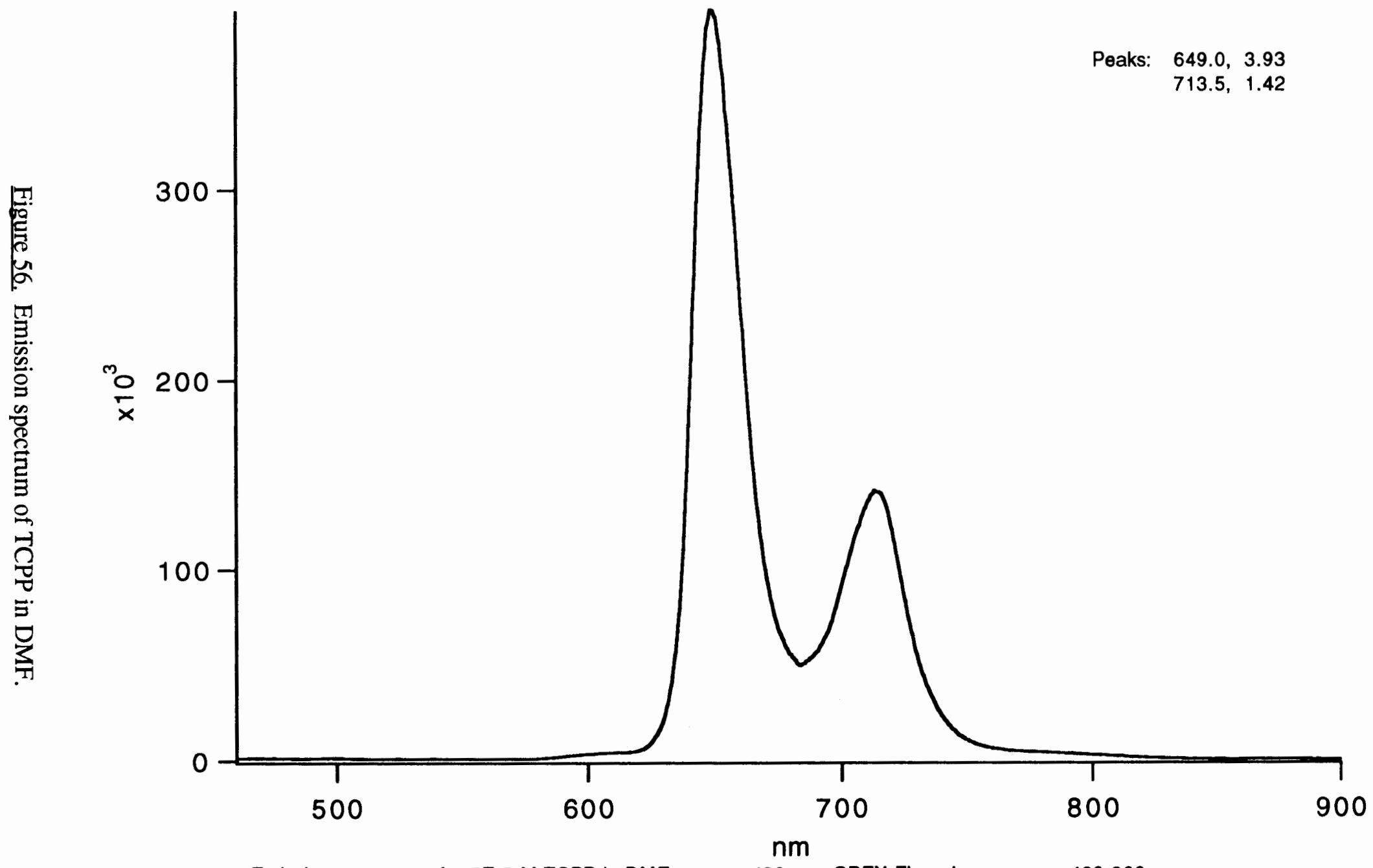

Emission spectrum of 2.5E-5 M TCPP in DMF; exc. at $432 \mathrm{~nm}$; SPEX Fluorolog; range $=460-900 \mathrm{~nm}$

PMT voltages $=950 / 120 \mathrm{~V} ; 1 \mathrm{~cm}$ quartz cell; corrected for blank; acq. $\operatorname{mode}=6$ (KS1-S2); $0.5 \mathrm{~nm}$ incr.;

1 sec. integ. time; exc. slits $=5.0 \mathrm{~mm}$; em. slits $=0.5 \mathrm{~mm}$; right angle detection; Date: $7-7-94 ;$

lgor expt. "Emission" 


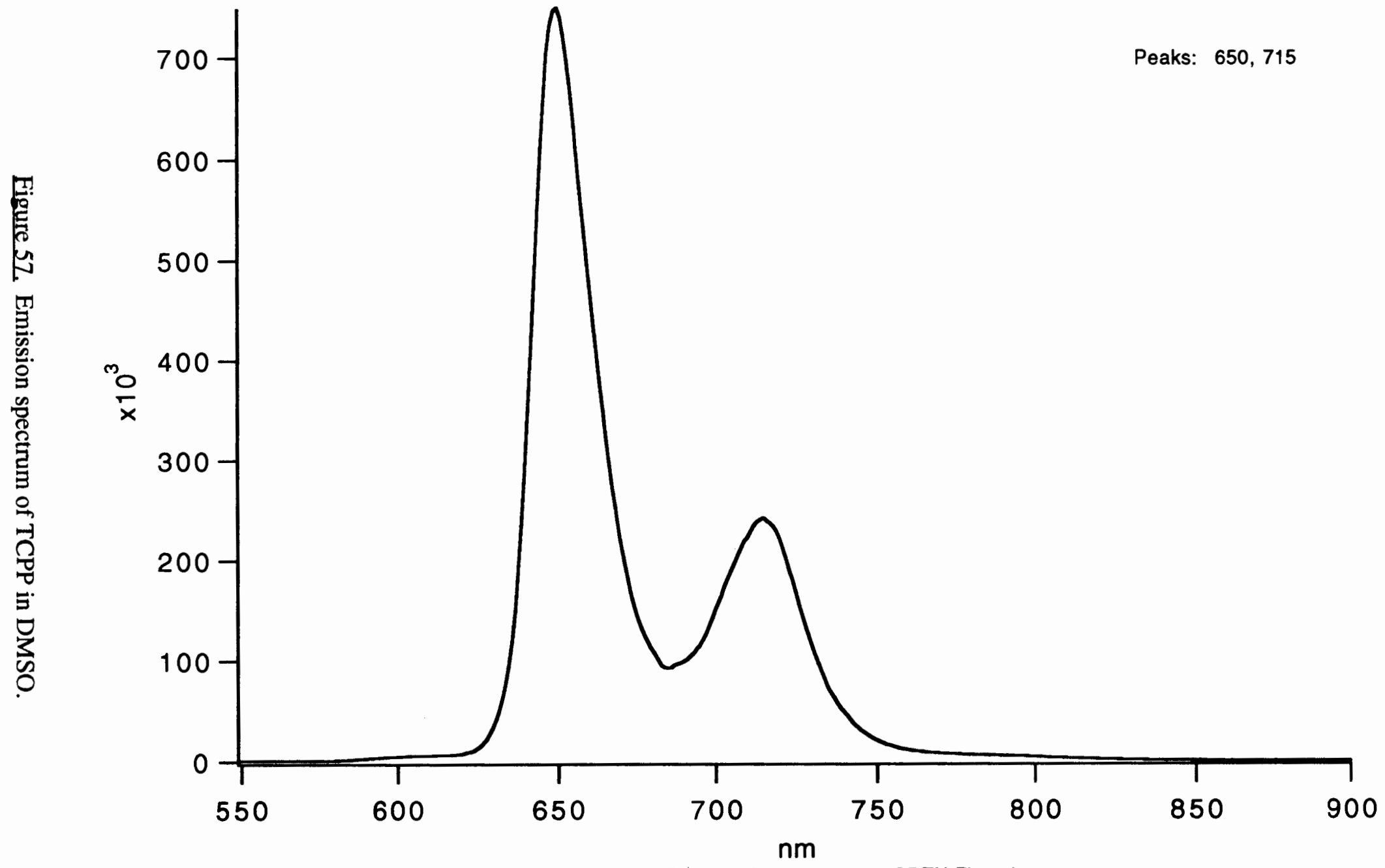

Emission spectrum of 1.00e-6 M TCPP in DMSO; excitation at $422 \mathrm{~nm}$; SPEX Fluorolog;

range $=550-900 ; 1 \mathrm{~nm}$ incr.; $1 \mathrm{sec}$. integ. time; PMT voltages = 990/120; acq. mode = 6 (KS1-S2);

exc. slits $=5.0 \mathrm{~mm} ;$ em. slits $=0.5 \mathrm{~mm} ; 1 \mathrm{~cm}$ quartz cell; right angle detection; corrected for blank;

Igor expt. "Emission"; NB2 p. 28; Date: 5-22-95 


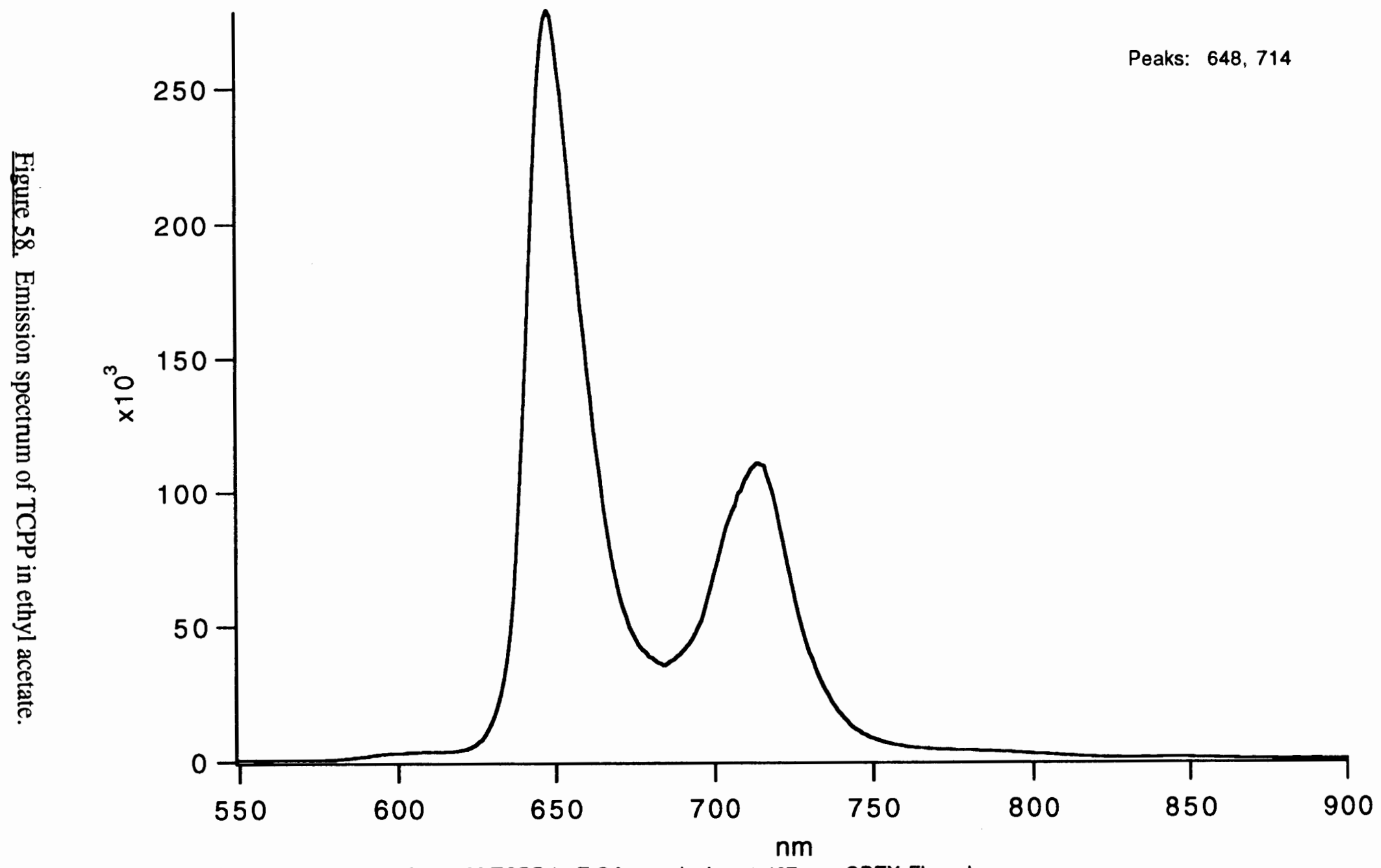

Emission spectrum of 10-6 M TCPP in EtOAc; excitation at $427 \mathrm{~nm}$; SPEX Fluorolog;

range $=550-900 ; 1 \mathrm{~nm}$ incr.; 1 sec. integ. time; PMT voltages = 990/0; acq. mode $=7(\mathrm{~S} 1)$;

exc. slits $=5.0 \mathrm{~mm}$; em. slits $=0.5 \mathrm{~mm} ; 1 \mathrm{~cm}$ quartz cell; right angle detection; not corrected for blank;

Igor expt. "Emission"; NB p. 94; Date: 2-13-95 


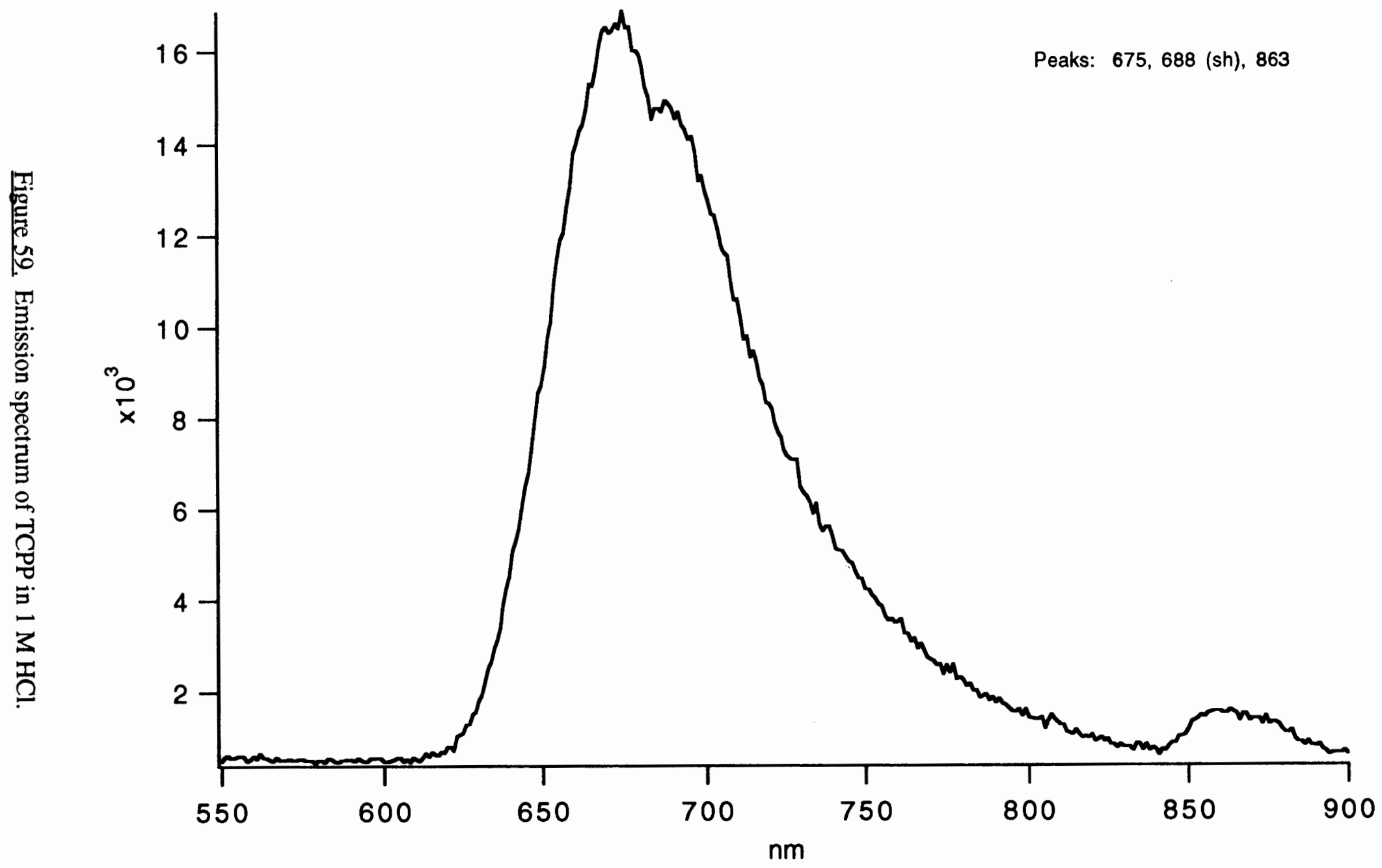

Emission spectrum of 2e-6 M TCPP in $1 \mathrm{M} \mathrm{HCl}$; excitation at $438 \mathrm{~nm}$; SPEX Fluorolog;

range $=550-900 ; 1 \mathrm{~nm}$ incr.; 1 sec. integ. time; PMT voltages $=990 / 0$; acq. mode $=0(S 1)$;

exc. slits $=5.0 \mathrm{~mm}$; em. slits $=0.5 \mathrm{~mm} ; 1 \mathrm{~cm}$ quartz cell; right angle detection; not corrected for blank;

Igor expt. "Emission"; NB2 p. 12; Date: 4-13-95 


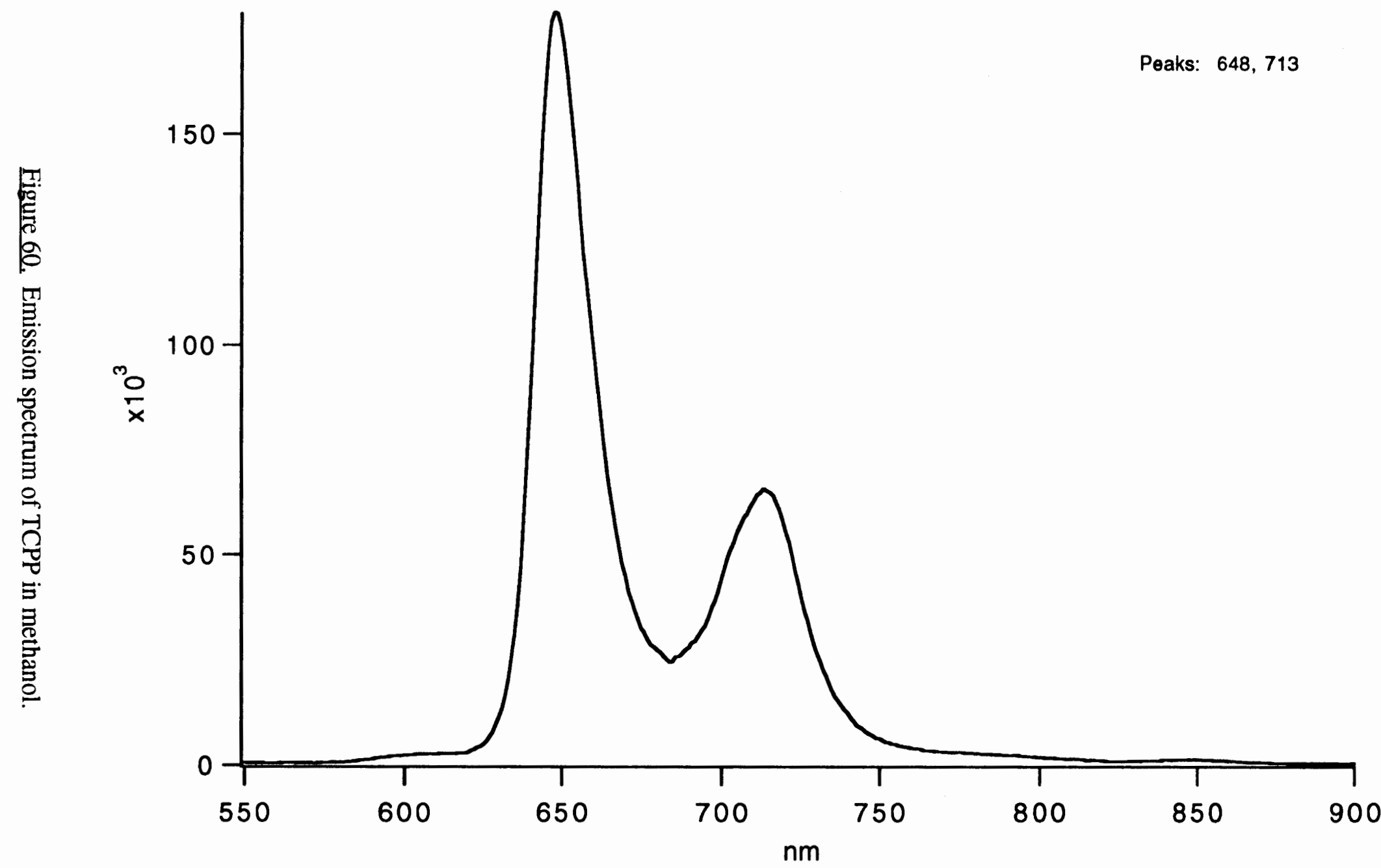

Emission spectrum of 1e-6 M TCPP in $\mathrm{MeOH}$; excitation at $427 \mathrm{~nm}$; SPEX Fluorolog;

range $=550-900 ; 1 \mathrm{~nm}$ incr.; 1 sec. integ. time; PMT voltages $=990 / 0 ;$ acq. mode $=7$ (S1);

exc. slits $=5.0 \mathrm{~mm} ;$ em. slits $=0.5 \mathrm{~mm} ; 1 \mathrm{~cm}$ quartz cell; right angle detection; not corrected for blank:

Igor expt. "Emission"; NB p. 94; Date: 2-13-95 


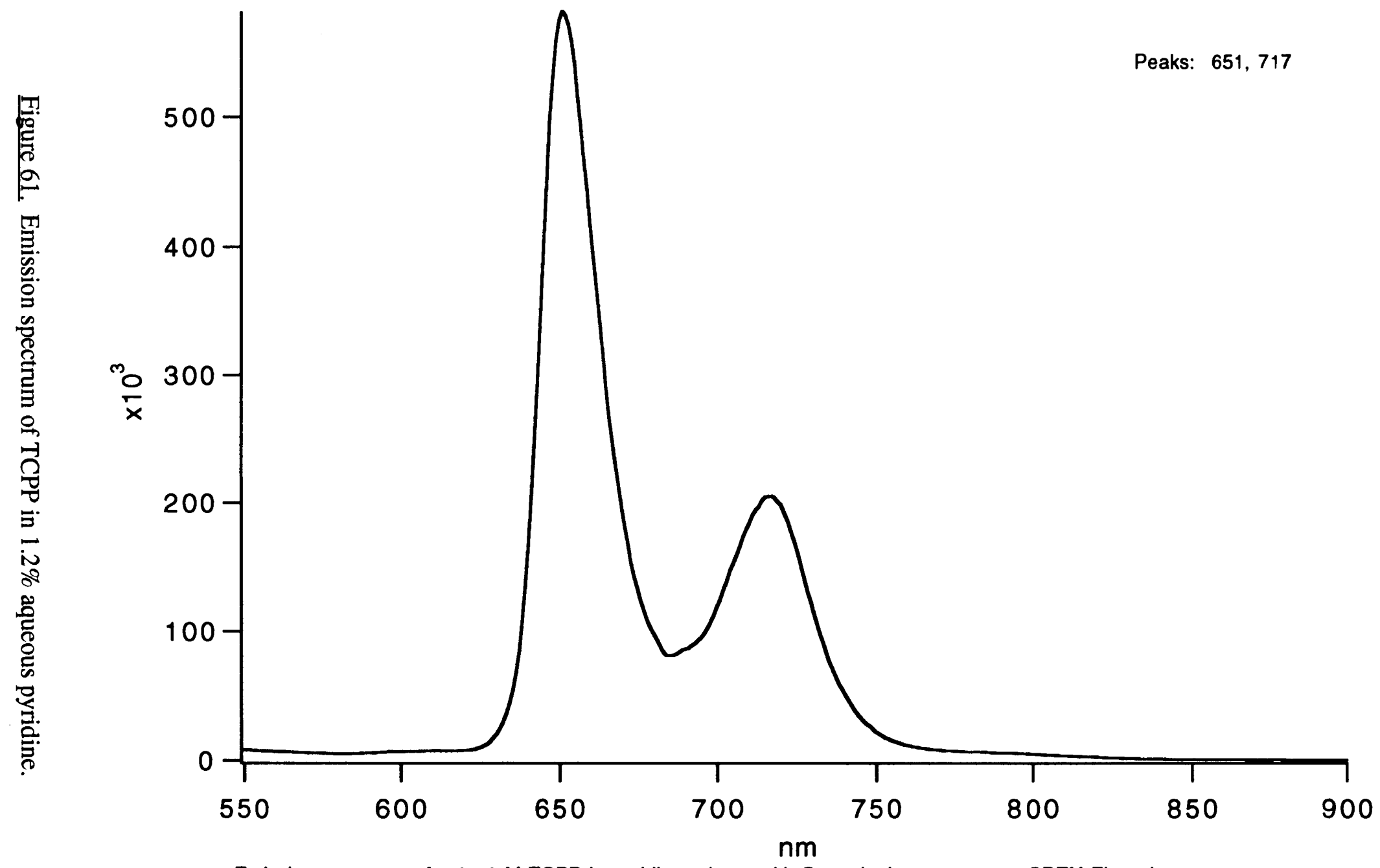

Emission spectrum of 1.0e-6 M TCPP in pyridine w/ 1.2\% H2O; excitation at $430 \mathrm{~nm}$; SPEX Fluorolog; range = 550-900; $1 \mathrm{~nm}$ incr.; 1 sec. integ. time; PMT voltages = 990/120; acq. mode = 6 (KS1-S2); exc. slits $=5.0 \mathrm{~mm}$; em. slits $=0.5 \mathrm{~mm} ; 1 \mathrm{~cm}$ quartz cell; right angle detection; corrected for blank; lgor expt. "Emission"; NB1 p. 72; Date: 12-18-94 


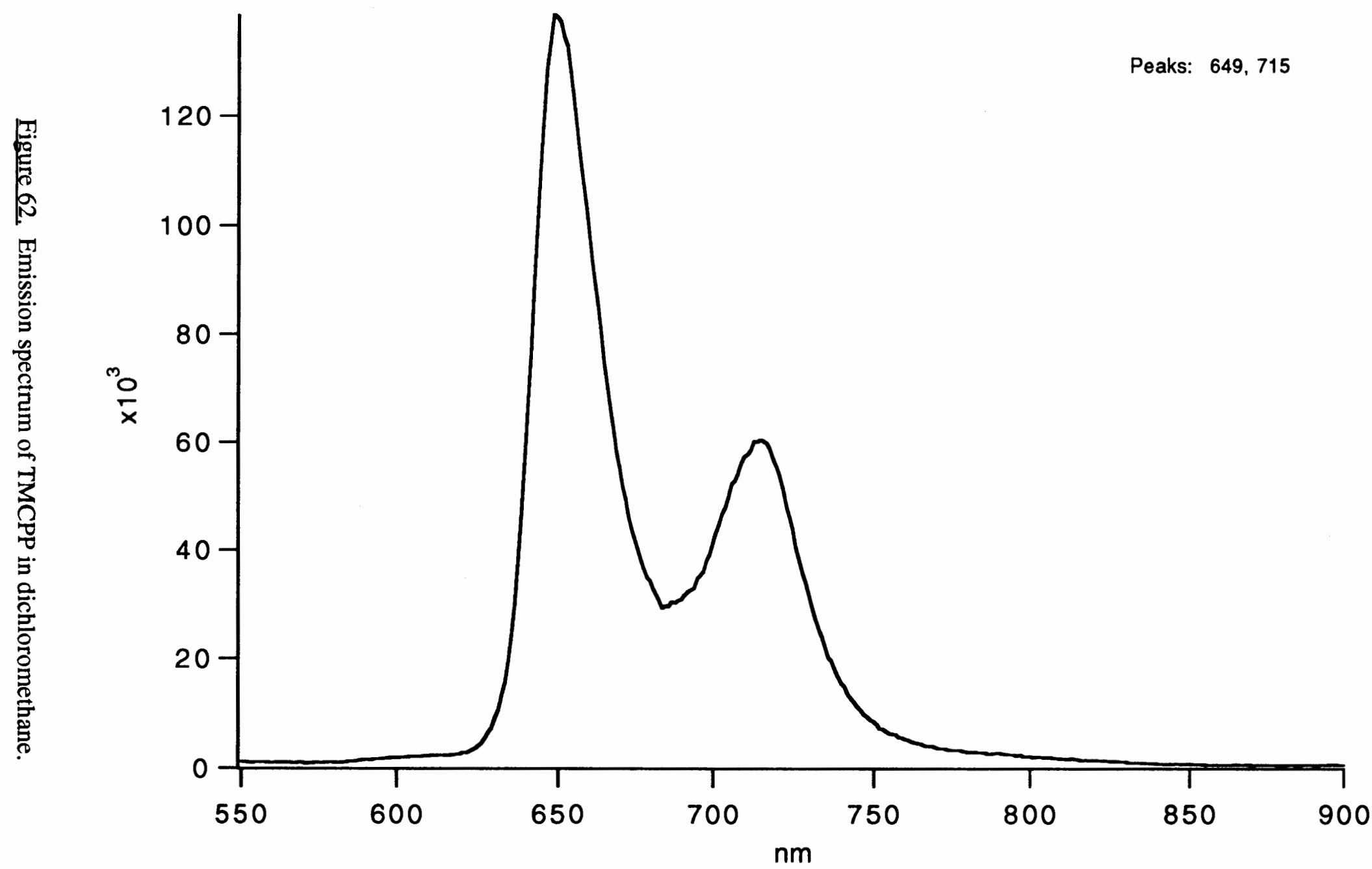

Emission spectrum of TCPP, tetrakis-methyl ester in DCM, unkn. conc.; excitation at $419 \mathrm{~nm}$; SPEX Fluorolog; range $=550-900 ; 1 \mathrm{~nm}$ incr.; 1 sec. integ. time; PMT voltages $=990 / 0 ;$ acq. mode $=0$ (S1);

exc. slits $=5.0 \mathrm{~mm} ;$ em. slits $=0.5 \mathrm{~mm} ; 1 \mathrm{~cm}$ quartz cell; right angle detection; not corrected for blank; Igor expt. "Emission"; NB1 p. 95; Date: 3-19-95 


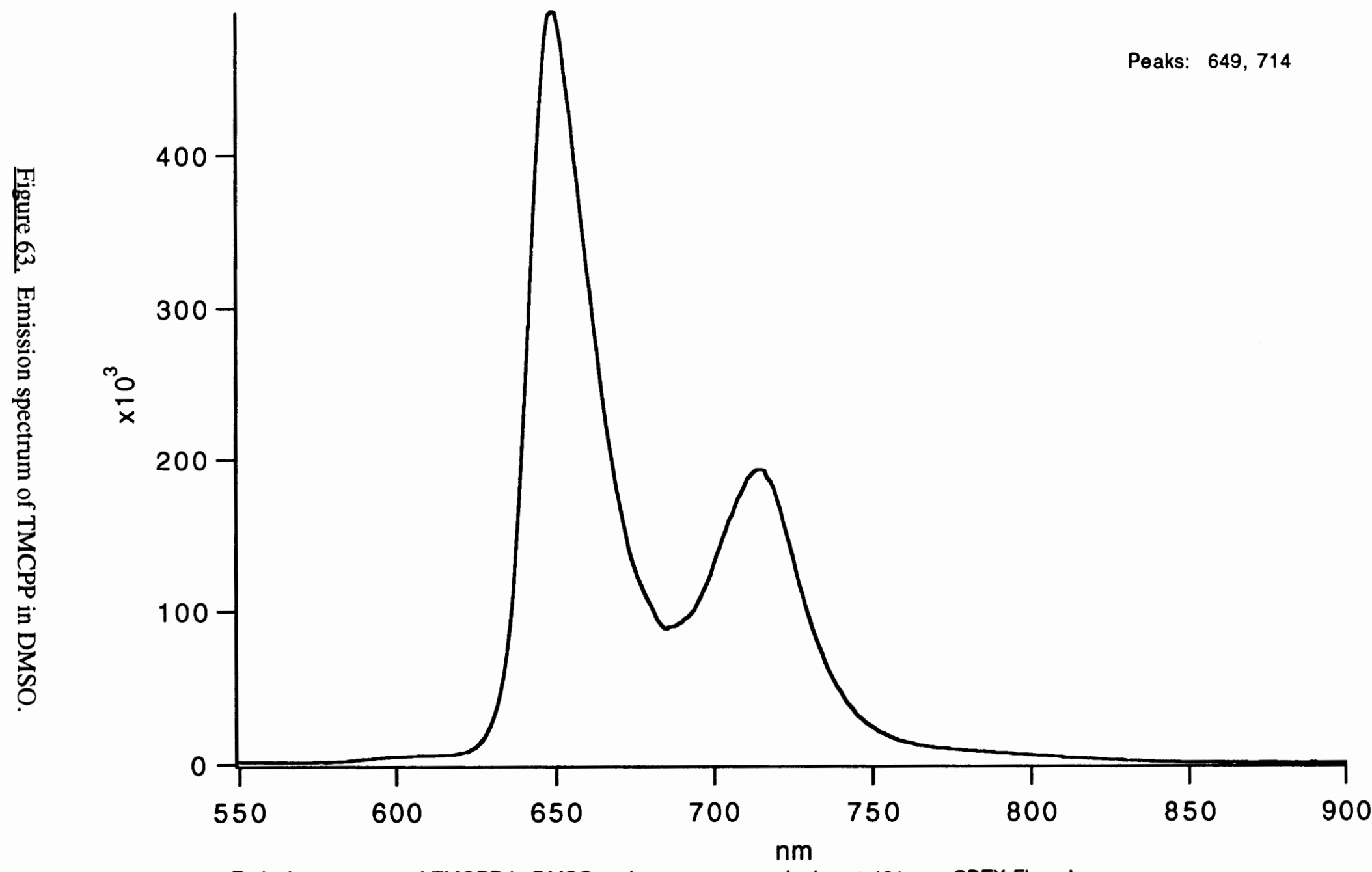

Emission spectrum of TMCPP in DMSO, unknown conc.; excitation at $421 \mathrm{~nm}$; SPEX Fluorolog;

range = 550-900; $1 \mathrm{~nm}$ incr.; 1 sec. integ. time; PMT voltages = 990/120; acq. mode = 6 (KS1-S2);

exc. slits $=5.0 \mathrm{~mm}$; em. slits $=0.5 \mathrm{~mm} ; 1 \mathrm{~cm}$ quartz cell; right angle detection; corrected for blank;

Igor expt. "Emission"; NB1 p. 86; Date: 1-25-95 


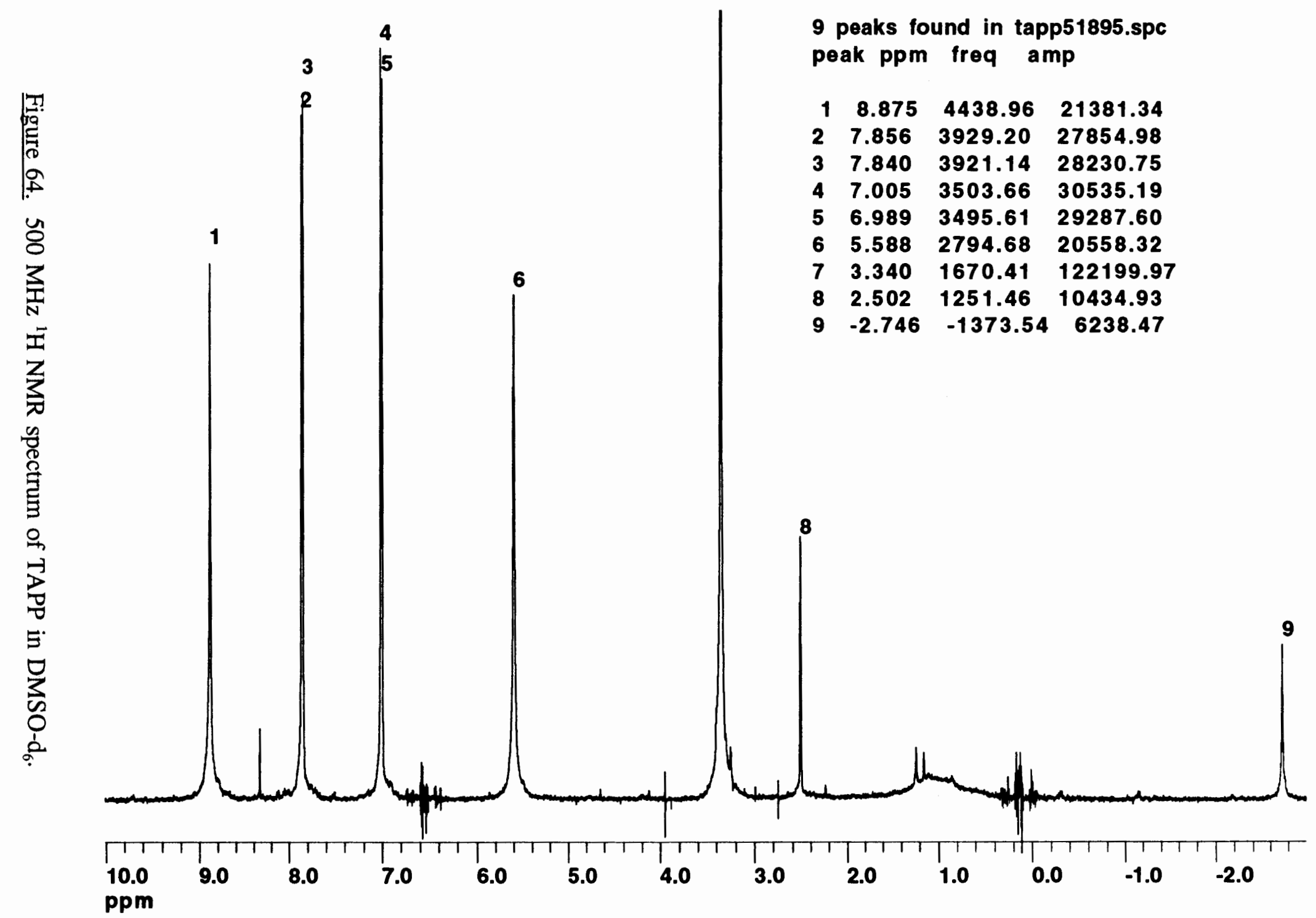


5 peaks found in tapp+D20_51895.spe peak ppm freq amp

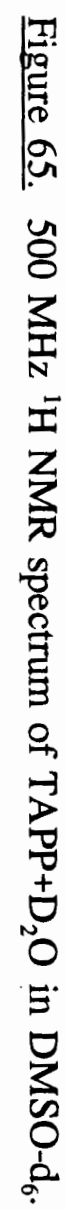

$\begin{array}{llll}1 & 8.291 & 4146.97 & 3361.82\end{array}$

$\begin{array}{llll}2 & 7.859 & 3930.91 & 27543.87\end{array}$

$\begin{array}{lllll}3 & 7.843 & 3922.85 & 28677.59\end{array}$

$4 \quad 7.020 \quad 3511.23 \quad 30759.59$

$\begin{array}{llllll}\text { in } & & 5 & 7.004 & 3503.17 & 29330.74\end{array}$

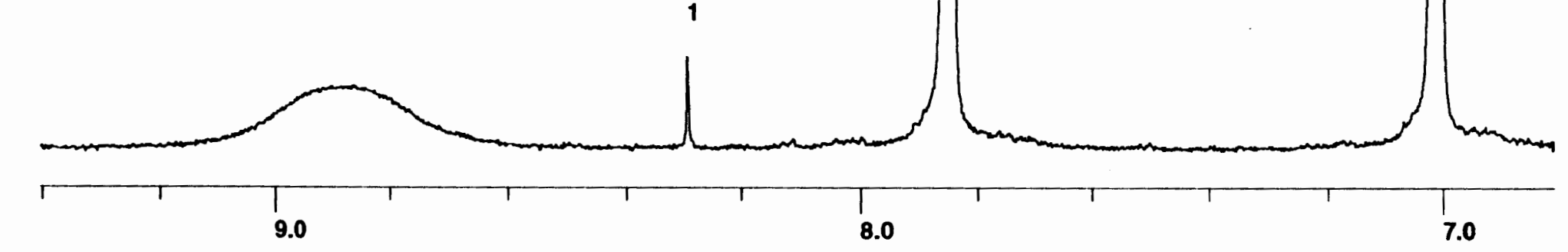

Ppm 


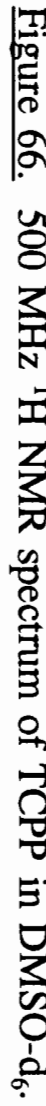

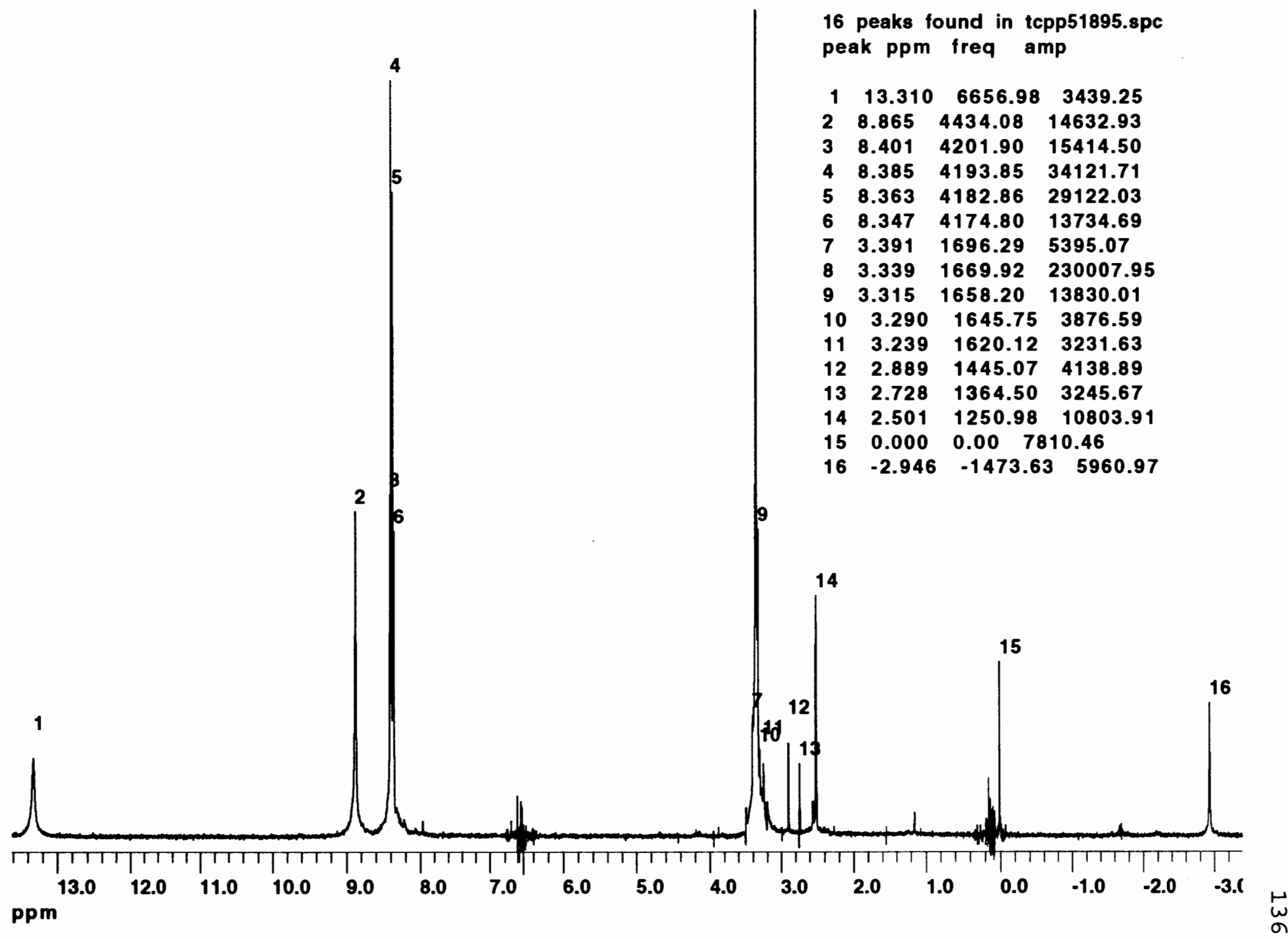


4 peaks found in tcpp+D2O_51895.spc peak ppm freq amp

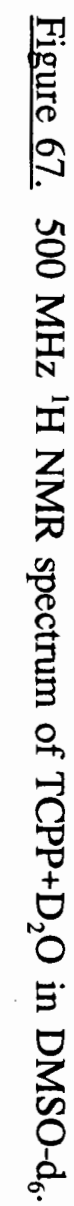

$\begin{array}{llll}1 & 8.407 & 4204.83 & 15868.21\end{array}$

$\begin{array}{lllll}2 & 8.391 & 4196.78 & 31888.18\end{array}$

$\begin{array}{llll}3 & 8.364 & 4183.59 & 27641.60\end{array}$

$\begin{array}{lllll}9 & 4 & 8.348 & 4175.54 & 14861.30\end{array}$

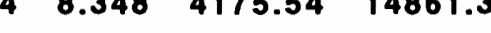

